\title{
25. SOUND VELOCITY, ELASTIC CONSTANTS, AND RELATED PROPERTIES OF MARINE SEDIMENTS IN THE WESTERN EQUATORIAL PACIFIC: LEG 7, GLOMAR CHALLENGER
}

\author{
E. L. Gealy, Scripps Institution of Oceanography, La Jolla, California
}

\begin{abstract}
The velocity of the compressional wave was measured with a shipboard velocimeter at three intervals along whole core sections 1.5 meters long for almost all sections of nonindurated and semi-indurated sediments recovered on Leg 7 of the D/V Glomar Challenger in the Western Equatorial Pacific. In addition, the compressional wave velocity was measured on selected samples of indurated materials with a velocimeter at the University of Hawaii. Disturbance by the coring process renders measurements on some core sections as unrepresentative of in situ conditions, but other measurements appear to approach the compressional wave velocity of in situ materials closely.
\end{abstract}

Measurements of compressional wave velocity, measurements of saturated bulk density and porosity reported in Chapter 25 , and estimates of bulk modulus are used to compute impedance, rigidity, Lame's constant, Poisson's ratio, and the velocity of the shear wave for almost all core sections recovered on Leg 7.

Holes at Sites 62, 63 and 64 penetrated sequences of nannofossil ooze, chalk and limestone as deep as 960 meters beneath the sea floor, and range in age from Eocene to Recent.

The calcareous ooze-chalk sequences at these sites show an irregular exponential increase in compressional wave velocities with depth. The rates of increase at a given depth are greatest at Site 63 and least at Site 64. Compressional wave velocities at all three sites are about 1.45 to $1.50 \mathrm{~km} / \mathrm{sec}$ at the surface. At 500 meters the compressional wave velocity is about 1.7 $\mathrm{km} / \mathrm{sec}$ at Site $64,2.1 \mathrm{~km} / \mathrm{sec}$ at Site 62 , and $2.3 \mathrm{~km} / \mathrm{sec}$ at Site 63. A silicified limestone from 950 meters at Site 64 had a compressional wave velocity of $4.5 \mathrm{~km} / \mathrm{sec}$.

Shear wave velocities at these sites also increase with depth, but the rate of increase decreases with depth. Shear wave velocities at Site 64 are lower throughout than those at Sites 62 and 63.

Insufficient reliable data were collected on this leg to determine velocity gradients in either radiolarian ooze or pelagic clay.

The upper 20 to 30 meters of the calcareous ooze at Sites 62 , 63 and 64 has a compressional wave velocity less than in seawater. Near surface sediments at Sites 65 and 66 also have compressional wave velocities less than that in seawater. Thus, a low velocity channel exists in these upper sediments.

The velocity at the compressional wave decreases as the fraction clay size increases where other parameters are the 
same. Cementation at Sites 62,63 and 64 tends to obliterate this relationship.

Measurements of compressional wave velocity parallel and perpendicular to bedding indicate that some anisotropy may exist in calcareous sediments. Samples having a horizontal velocity less than $2.0 \mathrm{~km} / \mathrm{sec}$ had a higher vertical velocity than horizontal; samples having a horizontal velocity more than $2.0 \mathrm{~km} / \mathrm{sec}$ had a lower vertical velocity than horizontal. In unconsolidated and semiconsolidated calcareous deposits, advective fluid may cause a vertical grain to develop; compaction may destroy this grain in indurated sediments, crushing grain horizontal layers.

\section{INTRODUCTION}

Compressional wave velocities were measured on sediment cores and samples recovered on Leg 7; and, these data, together with saturated bulk density measurements, permit derivation of several other elastic constants for marine sediment sections in the Western Equatorial Pacific.

\section{METHODS}

Because the acoustic properties of rocks depend on vectoral properties which are changed by removing a sample from its in situ environment to the laboratory, it is important to obtain sound velocity measurements in situ if possible. Down-hole acoustic logging devices exist which can measure the velocity of the compressional wave in situ, but no such tools were available on Leg 7.

The only means of determining the velocity of the compressional wave on Leg 7 were: 1) by comparing travel time to prominent reflectors on seismic profiling records with driller's depths to a change in lithology that might cause a change in acoustic impedance, and 2) by measuring the velocity of the compressional wave in core sections in the laboratory. Seismic profiling records are discussed elsewhere in this volume. Measurements of the velocity of the compressional wave were made on almost all core sections retrieved on Leg 7 of the D/V Glomar Challenger by techniques described below. These measurements, together with measurements of some related properties, provide the basis of this study. Values of saturated bulk density and porosity used in this chapter are from studies reported in Gealy (1971).

\section{Instrumentation and Laboratory Techniques}

Aboard the D/V Glomar Challenger on Leg 7, some velocity measurements were made on unsplit core sections using an Underwater Systems Laboratory Sonic Velocimeter. Velocity measurements were commonly made at three places along each 150 . centimeter section if the condition of the core indicated that measurements might be valid. In general, velocity measurements were omitted on sections either partially empty or containing much water.

The device consists of a pulse generator emitting a 250 volt pulse of about 0.5 microseconds which excites a barium titanate transducer to emit compressional pulses at a natural resonant frequency of about 400 $\mathrm{kHz}$. The transmitted pulse triggers an oscilloscope which displays the incoming signals.

The velocity of the compressional wave in the sediment is determined from the difference between the measured transmission time across the diameter of the whole core section through the liner, and across a reference sample of liner filled with distilled water. Details of the equipment and procedures are outlined in DSDP Volume II in the Initial Core Descriptions, (Peterson et. al., 1969).

The temperature of each core section was measured at the time that velocity was measured, and values ranged from $22^{\circ} \mathrm{C}$ to $27.4^{\circ} \mathrm{C}$. All values of velocity were corrected to $23^{\circ} \mathrm{C}$ by

$$
\frac{\mathrm{V}_{\mathrm{p}}}{\mathrm{V}_{\mathrm{p}}^{\prime}}=\frac{\mathrm{V}_{\mathrm{w}}}{\mathrm{V}_{\mathrm{w}}{ }^{\prime}}
$$

$$
\begin{aligned}
& \text { where } \mathrm{V}_{\mathrm{p}}=\text { velocity of compressional wave cor- } \\
& \mathrm{V}_{\mathrm{p}}^{\prime}=\text { velocity of compressional wave at } \\
& \mathrm{V}_{\mathrm{w}}=\text { velocity of seawater at } 35 \% \text { salinity }
\end{aligned}
$$

The velocimeter described above is designed to measure the velocity of the compressional wave only in unconsolidated and semiconsolidated sediments in plastic core liners. 
Also aboard the D/V Glomar Challenger on Leg 7 was a microtrans device designed to measure the velocity of the compressional wave in indurated rock samples. Details of the instrumentation and procedures are given in Volume II of the Initial Reports of the Deep Sea Drilling Project. On Leg 7 this device consistently failed to yield meaningful results.

In order to supplement shipboard measurements, the velocity of the compressional wave was determined in selected oriented samples of indurated materials from all sites, except Site 61, by a velocimeter at the Core Analysis Laboratory of the Hawaii Institute of Geophysics, University of Hawaii.

\section{Sources of Error}

Measurements of the velocity of the compressional wave by these laboratory techniques may differ from in situ velocities substantially.

First, simply by removing the sediment to the laboratory and thus from its in-situ temperature, pore pressure and confining pressure, changes the velocity $\left(\mathrm{V}_{\mathrm{p}}\right)$.

Second, the drilling and coring process disturbs the cored materials (Chapter 2, this volume) and, in some cases, completely destroys any rigidity the rock may have had. The coring process may cause either an increase or a decrease in porosity, although on this leg an increase in porosity is more common. Thus, such disturbances commonly result in a reduction in velocity $\left(\mathrm{V}_{\mathrm{p}}\right)$.

On Sites 62, 63 and 64, when soft nannofossil chalk was cored, the drilling rhythm caused the recovery of a series of "biscuits" of undisturbed chalk separated by variable thicknesses of pulverized chalk paste. Commonly the chalk biscuits would be fractured, and cores received in this condition were of doubtful value for sonic velocity measurements using the shipboard velocimeter. The fractured part of the original chalk would not transmit the sonic pulse at all, and no reading could be taken there. The paste would transmit the pulse between the transducer head pads, but, of course, it was not representative of the actual rock. While processing Core 8 of Hole 63.0, Heath and Moberley attempted to make sonic measurements along the entire length of Section 2, rather than only at the three standard places. The unopened core liner was marked where no signal was transmitted across the core. Subsequently, when the section was split it could be seen that each of the nine segments marked as worthless for velocity measurements were segments of chalk, whereas all the measurements that were taken were across mud. The observations were confirmed on several additional cores.
Third, the geometry of the cores and the measuring equipment may result in errors. The outside diameter of the core liners varies from section to section, and the velocity measurements are diameter dependent. The thickness of the liner wall varies from section to section and is not measured. All cores retrieved contain a slurry of diluted, homogenized sediment paste between the liner and the core proper. The thickness of this slurry is variable, but is commonly 1 to 5 millimeters thick.

Because most of these factors tend to result in measurements less than the in situ velocity, except where otherwise noted, it has been assumed that the maximum of the three compressional velocity measurements made on any section most closely approximates the true in situ velocity. It is these maximum measurements $\left[\mathrm{V}_{\mathrm{p}}(\max ).\right]$ which are listed in the tables in Chapters 2 through 4 and are plotted as a function of depth on the core sheets and site summaries in those chapters, and on Figures 1 through 6 in this chapter. In this chapter, these maximum values are listed in Tables 1 through 6, and are shown plotted as a function of depth on Figures 1 through 6.

\section{DEFINITIONS AND RELATIONSHIPS}

The following definitions and relationships are used in this study; the values of all properties are for the laboratory ( 1 atmosphere pressure), and $23^{\circ} \mathrm{C}$ :

Saturated Bulk Density and Porosity

$$
\rho_{\mathrm{B}}=\phi \rho_{\mathrm{F}}+(1-\phi) \rho_{\mathrm{G}}
$$

where: $\phi=$ porosity: that fraction of total volume of sediment in its fully saturated state, not occupied by solid components,

$\rho=$ density in grams per cubic centimeter of the interstitial fluid in a sediment core in its fully saturated state at its natural salinity,

$\rho_{\mathrm{G}}=$ weighted mean bulk density in grams per cubic centimeter of the solid component of a sediment core, excluding solids dissolved in the interstitial water.

Values of Saturated Bulk Density and Porosity (Gealy, 1971) are listed in Tables 1 through 6 and are shown plotted as a function of depth on Figures 1 through 6.

\section{Velocity Compressional Wave}

For purposes of this study, it is assumed that a compressional wave moving in saturated sediments is of such small amplitude and short duration that its passage results in no loss of fluids and no permanent 
TABLES 1 through 6

Sound Velocity, Elastic Constants and Related Properties

\begin{tabular}{l}
\hline \multicolumn{2}{l}{ Identification } \\
Hole Core
\end{tabular}

Table 1. Site 61

$\begin{array}{llllllllllllllll}61.1 & 1 & 2 & \text { Porcelanite, mudstone } & 85.25 & 1.98 & 40.8 & 1.726 & 3419 & 37.700 & 1.621 & 6.581 & 0.000 & 6.581 & 0.500 & 0.000\end{array}$ shale and siltstone

Table 2. Site 62

\begin{tabular}{|c|c|c|c|c|c|c|c|c|c|c|c|c|c|c|c|}
\hline 62.0 & 1 & 1 & Nannofossil chalk ooze & 91.75 & 1.66 & 60.4 & 1.590 & 2646 & 67.584 & 0.259 & 4.108 & 0.094 & 4.045 & 0.489 & 0.238 \\
\hline 62.0 & 1 & 2 & Nannofossil chalk ooze & 93.25 & 1.67 & 61.8 & 1.679 & 2801 & 66.703 & 0.227 & 3.996 & 1.097 & 3.264 & 0.374 & 0.811 \\
\hline 62.0 & 1 & 3 & Nannofossil chalk ooze & 94.75 & 1.68 & 60.8 & 1.616 & 2721 & 68.535 & 0.249 & 4.076 & 0.242 & 3.915 & 0.471 & 0.379 \\
\hline 62.0 & 1 & 4 & Nannofossil chalk ooze & 96.25 & 1.66 & 62.3 & 1.601 & 2658 & 67.584 & 0.217 & 3.961 & 0.236 & 3.804 & 0.471 & 0.377 \\
\hline 62.0 & 1 & 5 & Nannofossil chalk ooze & 97.75 & 1.66 & 62.4 & 1.595 & 2643 & 65.645 & 0.214 & 3.947 & 0.273 & 3.764 & 0.466 & 0.406 \\
\hline 62.0 & 1 & 6 & Nannofossil chalk ooze & 99.25 & 1.65 & 62.9 & 1.602 & 2641 & 67.865 & 0.205 & 3.916 & 0.249 & 3.750 & 0.469 & 0.389 \\
\hline 62.0 & 2 & 2 & Nannofossil chalk ooze & 207.25 & 1.73 & 58.4 & 1.729 & 2982 & 69.275 & 0.311 & 4.280 & 0.884 & 3.691 & 0.403 & 0.716 \\
\hline 62.0 & 2 & 3 & Nannofossil chalk ooze & 208.75 & 1.73 & 58.4 & 1.678 & 2896 & 68.359 & 0.314 & 4.285 & 0.470 & 3.972 & 0.447 & 0.522 \\
\hline 62.0 & 2 & 4 & Nannofossil chalk ooze & 210.25 & 1.72 & 58.5 & 1.722 & 2958 & 69.240 & 0.310 & 4.277 & 0.676 & 3.826 & 0.425 & 0.626 \\
\hline 62.0 & 2 & 5 & Nannofossil chalk ooze & 211.75 & 1.73 & 58.1 & 1.675 & 2900 & 69.733 & 0.322 & 4.315 & 0.441 & 4.021 & 0.451 & 0.505 \\
\hline 62.0 & 2 & 6 & Nannofossil chalk ooze & 213.25 & 1.73 & 58.2 & 1.704 & 2945 & 70.755 & 0.317 & 4.301 & 0.538 & 3.942 & 0.440 & 0.558 \\
\hline 62.0 & 3 & 1 & Nannofossil chalk ooze & 299.75 & 1.75 & 57.2 & 1.829 & 3194 & 69.768 & 0.350 & 4.396 & 1.189 & 3.603 & 0.376 & 0.825 \\
\hline 62.0 & 3 & 2 & Nannofossil chalk ooze & 301.25 & 1.72 & 58.6 & 1.752 & 3017 & 71.883 & 0.307 & 4.272 & 1.032 & 3.584 & 0.388 & 0.774 \\
\hline 62.0 & 3 & 3 & Nannofossil chalk ooze & 302.75 & 1.73 & 57.9 & 1.829 & 3169 & 68.923 & 0.326 & 4.324 & 1.201 & 3.523 & 0.373 & 0.832 \\
\hline 62.0 & 3 & 4 & Nannofossil chalk ooze & 304.25 & 1.74 & 57.6 & 1.808 & 3143 & 69.698 & 0.337 & 4.358 & 1.147 & 3.594 & 0.379 & 0.812 \\
\hline 62.0 & 3 & 5 & Nannofossil chalk ooze & 305.75 & 1.77 & 55.8 & 1.854 & 3279 & 70.826 & 0.398 & 4.535 & 1.278 & 3.683 & 0.371 & 0.850 \\
\hline 62.0 & 3 & 6 & Nannofossil chalk ooze & 307.25 & 1.73 & 58.1 & 1.848 & 3197 & 68.817 & 0.321 & 4.308 & 1.478 & 3.323 & 0.346 & 0.924 \\
\hline 62.0 & 4 & 1 & $\begin{array}{l}\text { Nannofossil chalk ooze } \\
\text { and chalk }\end{array}$ & 395.75 & 1.75 & 56.7 & 1.968 & 3452 & 65.293 & 0.366 & 4.434 & 1.916 & 3.157 & 0.311 & 1.045 \\
\hline 62.0 & 4 & 2 & $\begin{array}{l}\text { Nannofossil chalk ooze } \\
\text { and chalk }\end{array}$ & 397.25 & 1.88 & 49.6 & 1.904 & 3571 & 67.901 & 0.714 & 5.289 & 1.169 & 4.509 & 0.397 & 0.790 \\
\hline 62.0 & 4 & 3 & Nannofossil chalk ooze & 398.75 & 1.87 & 50.1 & 2.040 & 3805 & 66.879 & 0.678 & 5.207 & 2.258 & 3.702 & 0.311 & 1.100 \\
\hline
\end{tabular}
and chalk 


\begin{tabular}{|c|c|c|c|c|c|c|c|c|c|c|c|c|c|c|c|}
\hline 62.0 & 4 & 4 & $\begin{array}{l}\text { Nannofossil chalk ooze } \\
\text { and chalk }\end{array}$ & 400.25 & 1.70 & 59.9 & 1.911 & 3249 & 66.244 & 0.272 & 4.149 & 1.682 & 3.027 & 0.321 & 0.995 \\
\hline 62.0 & 4 & 5 & $\begin{array}{l}\text { Nannofossil chalk ooze } \\
\text { and chalk }\end{array}$ & 401.75 & 1.83 & 52.2 & 1.926 & 3524 & 67.125 & 0.558 & 4.935 & 1.459 & 3.962 & 0.365 & 0.893 \\
\hline 62.0 & 4 & 6 & $\begin{array}{l}\text { Nannofossil chalk ooze } \\
\text { and chalk }\end{array}$ & 403.25 & 1.74 & 57.6 & 1.939 & 3369 & 65.786 & 0.336 & 4.347 & 1.949 & 3.048 & 0.305 & 1.059 \\
\hline 62.0 & 5 & 2 & $\begin{array}{l}\text { Nannofossil chalk ooze } \\
\text { and chalk }\end{array}$ & 492.25 & 1.86 & 50.3 & 1.999 & 3723 & 69.099 & 0.666 & 5.189 & 1.818 & 3.976 & 0.343 & 0.988 \\
\hline 62.0 & 5 & 3 & $\begin{array}{l}\text { Nannofossil chalk ooze } \\
\text { and chalk }\end{array}$ & 493.75 & 1.90 & 48.2 & 2.020 & 3834 & 68.923 & 0.811 & 5.499 & 2.070 & 4.119 & 0.333 & 1.044 \\
\hline 62.0 & 5 & 4 & $\begin{array}{l}\text { Nannofossil chalk ooze } \\
\text { and chalk }\end{array}$ & 495.25 & 1.95 & 44.9 & 2.165 & 4227 & 70.368 & 1.099 & 6.066 & 3.168 & 3.955 & 0.278 & 1.274 \\
\hline 62.1 & 1 & 2 & $\begin{array}{l}\text { Foraminiferal nanno- } \\
\text { fossil chalk ooze }\end{array}$ & 8.25 & 1.42 & 76.8 & 1.545 & 2185 & 59.584 & 0.056 & 3.134 & 0.252 & 2.965 & 0.461 & 0.422 \\
\hline 62.1 & 1 & 3 & $\begin{array}{l}\text { Foraminiferal nanno- } \\
\text { fossil chalk ooze }\end{array}$ & 9.75 & 1.51 & 71.1 & 1.510 & 2282 & 57.611 & 0.096 & 3.401 & 0.113 & 3.325 & 0.484 & 0.273 \\
\hline 62.1 & 2 & 2 & $\begin{array}{l}\text { Foraminiferal nanno- } \\
\text { fossil chalk ooze }\end{array}$ & 17.25 & 1.50 & 71.8 & 1.504 & 2256 & & & & & & & \\
\hline 62.1 & 2 & 3 & $\begin{array}{l}\text { Foraminiferal nanno- } \\
\text { fossil chalk ooze }\end{array}$ & 18.75 & 1.53 & 69.7 & 1.502 & 2305 & & & & & & & \\
\hline 62.1 & 2 & 4 & $\begin{array}{l}\text { Foraminiferal nanno- } \\
\text { fossil chalk ooze }\end{array}$ & 20.25 & 1.53 & 70.0 & 1.480 & 2264 & & & & & & & \\
\hline 62.1 & 4 & 1 & Nannofossil chalk ooze & 34.75 & 1.50 & 71.9 & 1.679 & 2517 & 63.143 & 0.089 & 3.367 & 0.686 & 2.910 & 0.405 & 0.676 \\
\hline 62.1 & 4 & 2 & Nannofossil chalk ooze & 36.25 & 1.52 & 70.6 & 1.542 & 2342 & 62.720 & 0.100 & 3.429 & 0.162 & 3.322 & 0.477 & 0.326 \\
\hline 62.1 & 4 & 3 & Nannofossil chalk ooze & 37.75 & 1.51 & 71.1 & 1.529 & 2311 & & & & & & & \\
\hline 62.1 & 4 & 4 & Nannofossil chalk ooze & 39.25 & 1.55 & 68.8 & 1.532 & 2373 & 65.822 & 0.118 & 3.531 & 0.121 & 3.451 & 0.483 & 0.279 \\
\hline 62.1 & 4 & 5 & Nannofossil chalk ooze & 40.75 & 1.58 & 67.3 & 1.693 & 2668 & 65.927 & 0.136 & 3.623 & 0.747 & 3.125 & 0.404 & 0.688 \\
\hline 62.1 & 4 & 6 & Nannofossil chalk ooze & 42.25 & 1.57 & 67.6 & 1.644 & 2582 & 64.412 & 0.132 & 3.601 & 0.643 & 3.172 & 0.416 & 0.640 \\
\hline 62.1 & 6 & 1 & Nannofossil marl ooze & 54.75 & 1.50 & 71.5 & 1.543 & 2328 & 55.355 & 0.092 & 3.376 & 0.171 & 3.261 & 0.475 & 0.338 \\
\hline 62.1 & 6 & 2 & Nannofossil chalk ooze & 56.25 & 1.51 & 71.2 & 1.529 & 2308 & 66.244 & 0.094 & 3.403 & 0.149 & 3.303 & 0.478 & 0.315 \\
\hline 62.1 & 6 & 3 & Nannofossil chalk ooze & 57.75 & 1.52 & 70.9 & 1.548 & 2346 & 63.637 & 0.098 & 3.419 & 0.208 & 3.279 & 0.470 & 0.371 \\
\hline
\end{tabular}

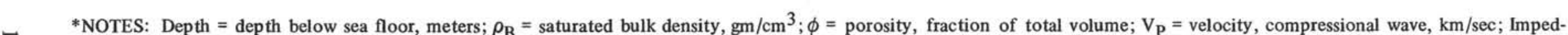
ance $=\mathrm{gm} / \mathrm{cm}^{2} \sec \times 10^{2} ; \mathrm{K}_{\mathrm{G}}=$ weighted mean bulk modulus, dynes $/ \mathrm{cm}^{2} \times 10^{10} ; \mathrm{K}_{\mathrm{S}}=$ skeletal bulk modulus, dynes $/ \mathrm{cm}^{2} \times 10^{10} ; \mathrm{K}=$ bulk modulus sediment, dynes $/ \mathrm{cm}^{2} \times$ $10^{10} ; \mu=$ rigidity, dynes $/ \mathrm{cm}^{2} \times 10^{10} ; \lambda=$ Lame's constant, dynes $/ \mathrm{cm}^{2} \times 10^{10} ; \theta=$ Poisson's ratio; $\mathrm{V}_{\mathrm{S}}=$ velocity of shear wave in $\mathrm{km} / \mathrm{sec}$. 
TABLES 1 through 6 - Continued

\begin{tabular}{|c|c|c|c|c|c|c|c|c|c|c|c|c|c|c|c|}
\hline \multicolumn{3}{|c|}{ Identification } & \multirow[b]{2}{*}{ Lithology } & \multirow[b]{2}{*}{ Depth* } & \multirow[b]{2}{*}{$\rho_{\mathrm{B}}$} & \multirow[b]{2}{*}{$\phi$} & \multirow[b]{2}{*}{$\mathrm{V}_{\mathrm{P}}$} & \multirow{2}{*}{$\begin{array}{l}\text { Acoustic } \\
\text { Impedance }\end{array}$} & \multirow[b]{2}{*}{$\mathrm{K}_{\mathrm{G}}$} & \multirow[b]{2}{*}{$\mathrm{K}_{\mathrm{S}}$} & \multirow[b]{2}{*}{ K } & \multirow[b]{2}{*}{$\mu$} & \multirow[b]{2}{*}{$\lambda$} & & \multirow[b]{2}{*}{$\mathrm{V}_{\mathrm{S}}$} \\
\hline Hole & Core & Section & & & & & & & & & & & & & \\
\hline 62.1 & 6 & 4 & Nannofossil marl ooze & 59.25 & 1.53 & 69.9 & 1.550 & 2373 & 58.527 & 0.106 & 3.462 & 0.194 & 3.332 & 0.472 & 0.356 \\
\hline 62.1 & 6 & 5 & Nannofossil chalk ooze & 60.75 & 1.54 & 69.6 & 1.570 & 2412 & 63.883 & 0.109 & 3.486 & 0.331 & 3.265 & 0.454 & 0.464 \\
\hline 62.1 & 6 & 6 & Nannofossil chalk ooze & 62.25 & 1.59 & 66.5 & 1.552 & 2465 & 64.482 & 0.146 & 3.667 & 0.155 & 3.564 & 0.479 & 0.313 \\
\hline 62.1 & 7 & 1 & Nannofossil chalk ooze & 63.75 & 1.55 & 68.8 & 1.555 & 2409 & 61.487 & 0.118 & 3.527 & 0.182 & 3.406 & 0.475 & 0.343 \\
\hline 62.1 & 7 & 2 & Nannofossil chalk ooze & 65.25 & 1.60 & 65.7 & 1.569 & 2515 & 60.606 & 0.159 & 3.718 & 0.175 & 3.601 & 0.477 & 0.331 \\
\hline 62.1 & 7 & 3 & Nannofossil chalk ooze & 66.75 & 1.57 & 67.9 & 1.543 & 2415 & 63.883 & 0.129 & 3.583 & 0.114 & 3.507 & 0.484 & 0.270 \\
\hline 62.1 & 7 & 4 & Nannofossil chalk ooze & 68.25 & 1.55 & 69.1 & 1.552 & 2397 & 59.478 & 0.115 & 3.511 & 0.197 & 3.380 & 0.472 & 0.357 \\
\hline 62.1 & 7 & 5 & Nannofossil chalk ooze & 69.75 & 1.63 & 64.4 & 1.548 & 2515 & 68.817 & 0.179 & 3.814 & 0.087 & 3.756 & 0.489 & 0.231 \\
\hline 62.1 & 7 & 6 & nalk ooze & & 1.57 & 67.5 & & & 64.835 & 0.133 & 3.606 & 0.108 & 3.535 & 0.485 & 0.262 \\
\hline 62.1 & 8 & 2 & Nannofossil chalk ooze & 74.25 & 1.62 & 64.5 & 1.537 & 2495 & 60.536 & 0.177 & 3.795 & 0.071 & 3.748 & 0.491 & 0.209 \\
\hline 62.1 & 8 & 3 & Nannofossil chalk ooze & 75.75 & 1.61 & 65.5 & 1.568 & 2516 & 61.698 & 0.161 & 3.728 & 0.198 & 3.596 & 0.474 & 0.351 \\
\hline 62.1 & 8 & 4 & Nannofossil chalk ooze & 77.25 & 1.63 & 64.2 & 1.543 & 2512 & 66.315 & 0.182 & 3.824 & 0.047 & 3.792 & 0.494 & 0.170 \\
\hline 62.1 & 8 & 5 & Nannofossil chalk ooze & 78.75 & 1.61 & 65.1 & 1.567 & 2526 & 63.637 & 0.167 & 3.756 & 0.178 & 3.637 & 0.477 & 0.333 \\
\hline 62.1 & 8 & 6 & Nannofossil chalk ooze & 80.25 & 1.63 & & & & & & & & & 0.491 & 0.208 \\
\hline 62.1 & 9 & 2 & Nannofossil chalk ooze & 83.25 & 1.60 & 65.9 & 1.546 & 2474 & 65.575 & 0.156 & 3.711 & 0.104 & 3.642 & 0.486 & 0.254 \\
\hline 62.1 & 9 & 2 & Nannofossil chalk ooze & 84.75 & 1.64 & 63.6 & 1.527 & 2501 & 63.778 & 0.193 & 3.863 & 0.043 & 3.834 & 0.495 & 0.162 \\
\hline 62.1 & 9 & 4 & Nannofossil chalk ooze & 86.25 & 1.59 & 66.3 & 1.565 & & 59.725 & 0.149 & 3.674 & 0.173 & 3.559 & 0.477 & 0.329 \\
\hline 62.1 & 10 & 1 & Nannofossil marl ooze & 92.75 & 1.58 & 67.3 & 1.510 & 2380 & 54.545 & 0.136 & 3.608 & 0.014 & 3.599 & 0.498 & 0.094 \\
\hline 62.1 & 10 & 2 & Nannofossil chalk ooze & 94.25 & 1.55 & 68.8 & 1.541 & 2388 & 64.482 & 0.118 & 3.532 & 0.170 & 3.418 & 0.476 & 0.331 \\
\hline 62.1 & 10 & 3 & Nannofossil chalk ooze & 95.75 & 1.58 & 67.3 & 1.543 & 2430 & 60.782 & 0.136 & 3.615 & 0.163 & 3.507 & 0.478 & 0.322 \\
\hline 62.1 & 10 & 4 & Nannofossil chalk ooze & 97.25 & 1.57 & 67.4 & 1.528 & 2404 & 64.800 & 0.135 & 3.613 & 0.088 & 3.554 & 0.488 & 0.237 \\
\hline 62.1 & 10 & 5 & Nannofossil chalk ooze & 98.75 & 1.59 & 66.5 & 1.573 & 2499 & 63.249 & 0.147 & 3.667 & 0.289 & 3.475 & 0.462 & 0.427 \\
\hline 62.1 & 10 & 6 & Nannofossil chalk ooze & 100.25 & 1.63 & 63.8 & 1.556 & 2542 & 64.835 & 0.188 & 3.846 & 0.136 & 3.755 & 0.483 & 0.289 \\
\hline 62.1 & 11 & 1 & Nannofossil chalk ooze & 101.75 & 1.65 & 62.9 & 1.603 & 2643 & 65.363 & 0.205 & 3.912 & 0.280 & 3.725 & 0.465 & 0.412 \\
\hline 62.1 & 11 & 2 & Nannofossil chalk ooze & 103.25 & 1.61 & 65.3 & 1.602 & 2577 & 65.363 & 0.164 & 3.749 & 0.340 & 3.522 & 0.456 & 0.460 \\
\hline 62.1 & 11 & 3 & Nannofossil chalk ooze & 104.75 & 1.63 & 64.0 & 1.563 & 2547 & 65.610 & 0.184 & 3.832 & 0.166 & 3.721 & 0.479 & 0.319 \\
\hline 62.1 & 11 & 4 & Nannofossil chalk ooze & 106.25 & 1.63 & 63.9 & 1.592 & 2599 & 61.980 & 0.187 & 3.838 & 0.245 & 3.674 & 0.469 & 0.388 \\
\hline 62.1 & 11 & 5 & Nannofossil chalk ooze & 107.75 & 1.62 & 64.4 & 1.614 & 2621 & 64.800 & 0.178 & 3.806 & 0.374 & 3.557 & 0.453 & 0.480 \\
\hline
\end{tabular}




\begin{tabular}{|c|c|c|c|c|c|c|c|c|c|c|c|c|c|c|c|}
\hline 62.1 & 11 & 6 & Nannofossil chalk ooze & 109.25 & 1.62 & 64.8 & 1.576 & 2549 & 65.046 & 0.171 & 3.778 & 0.287 & 3.587 & 0.463 & 0.421 \\
\hline 62.1 & 12 & 1 & Nannofossil chalk ooze & 110.75 & 1.62 & 64.5 & 1.553 & 2520 & 65.504 & 0.177 & 3.801 & 0.123 & 3.719 & 0.484 & 0.275 \\
\hline 62.1 & 12 & 2 & Nannofossil chalk ooze & 112.25 & 1.64 & 63.8 & 1.572 & 2569 & 67.161 & 0.189 & 3.854 & 0.159 & 3.748 & 0.480 & 0.312 \\
\hline 62.1 & 12 & 3 & Nannofossil chalk ooze & 113.75 & 1.67 & 61.6 & 1.622 & 2711 & 67.302 & 0.232 & 4.014 & 0.416 & 3.736 & 0.450 & 0.499 \\
\hline 62.1 & 12 & 4 & Nannofossil chalk ooze & 115.25 & 1.69 & 60.4 & 1.578 & 2669 & 67.125 & 0.260 & 4.112 & 0.120 & 4.032 & 0.486 & 0.266 \\
\hline 62.1 & 12 & 5 & Nannofossil chalk ooze & 116.75 & 1.65 & 62.9 & 1.587 & 2616 & 66.738 & 0.205 & 3.914 & 0.263 & 3.739 & 0.467 & 0.399 \\
\hline 62.1 & 12 & 6 & Nannofossil chalk ooze & 118.25 & 1.68 & 60.9 & 1.611 & 2709 & 68.077 & 0.246 & 4.066 & 0.245 & 3.903 & 0.471 & 0.382 \\
\hline 62.1 & 13 & 5 & Nannofossil chalk ooze & 125.75 & 1.62 & 64.6 & 1.637 & 2654 & 67.302 & 0.175 & 3.798 & 0.498 & 3.466 & 0.437 & 0.554 \\
\hline 62.1 & 14 & 2 & Nannofossil chalk ooze & 131.25 & 1.55 & 68.6 & 1.586 & 2465 & 67.478 & 0.121 & 3.549 & 0.311 & 3.341 & 0.457 & 0.447 \\
\hline 62.1 & 14 & 3 & Nannofossil chalk ooze & 132.75 & 1.63 & 64.3 & 1.599 & 2602 & 68.218 & 0.181 & 3.821 & 0.353 & 3.586 & 0.455 & 0.466 \\
\hline 62.1 & 14 & 4 & Nannofossil chalk ooze & 134.25 & 1.64 & 63.7 & 1.681 & 2750 & 65.117 & 0.190 & 3.854 & 0.601 & 3.453 & 0.426 & 0.606 \\
\hline 62.1 & 14 & 5 & Nannofossil chalk ooze & 135.75 & 1.59 & 66.4 & 1.618 & 2572 & 67.865 & 0.147 & 3.677 & 0.403 & 3.408 & 0.447 & 0.503 \\
\hline 62.1 & 14 & 6 & Nannofossil chalk ooze & 137.25 & 1.49 & 72.2 & 1.601 & 2390 & 67.407 & 0.086 & 3.354 & 0.437 & 3.063 & 0.438 & 0.541 \\
\hline 62.1 & 15 & 2 & Nannofossil chalk ooze & 140.25 & 1.66 & 62.3 & 1.650 & 2739 & 67.865 & 0.217 & 3.961 & 0.464 & 3.652 & 0.444 & 0.529 \\
\hline 62.1 & 15 & 3 & Nannofossil chalk ooze & 141.75 & 1.66 & 62.2 & 1.612 & 2677 & 68.218 & 0.219 & 3.967 & 0.262 & 3.793 & 0.468 & 0.397 \\
\hline 62.1 & 15 & 4 & Nannofossil chalk ooze & 143.25 & 1.66 & 62.1 & 1.648 & 2742 & 58.879 & 0.222 & 3.963 & 0.417 & 3.685 & 0.449 & 0.501 \\
\hline 62.1 & 15 & 5 & Nannofossil chalk ooze & 144.75 & 1.67 & 61.9 & 1.660 & 2765 & 61.522 & 0.225 & 3.978 & 0.477 & 3.660 & 0.443 & 0.535 \\
\hline 62.1 & 15 & 6 & Nannofossil chalk ooze & 146.25 & 1.68 & 61.3 & 1.619 & 2716 & 66.914 & 0.239 & 4.039 & 0.432 & 3.751 & 0.448 & 0.508 \\
\hline 62.1 & 16 & 2 & Nannofossil chalk ooze & 149.25 & 1.65 & 62.7 & 1.638 & 2708 & 68.887 & 0.209 & 3.933 & 0.500 & 3.599 & 0.439 & 0.550 \\
\hline 62.1 & 16 & 3 & Nahnofossil chalk ooze & 150.75 & 1.69 & 60.6 & 1.635 & 2760 & 68.288 & 0.255 & 4.095 & 0.434 & 3.805 & 0.449 & 0.507 \\
\hline 62.1 & 16 & 4 & Nannofossil chalk ooze & 152.25 & 1.70 & 59.8 & 1.620 & 2756 & 67.161 & 0.273 & 4.155 & 0.273 & 3.973 & 0.468 & 0.401 \\
\hline 62.1 & 16 & 5 & Nannofossil chalk ooze & 153.75 & 1.69 & 60.4 & 1.650 & 2791 & 69.134 & 0.259 & 4.111 & 0.397 & 3.847 & 0.453 & 0.484 \\
\hline 62.1 & 16 & 6 & Nannofossil chalk ooze & 155.25 & 1.73 & 58.4 & 1.649 & 2845 & 66.139 & 0.312 & 4.276 & 0.384 & 4.019 & 0.456 & 0.472 \\
\hline 62.1 & 17 & 2 & Nannofossil chalk ooze & 158.25 & 1.69 & 60.4 & 1.594 & 2698 & 68.147 & 0.260 & 4.111 & 0.214 & 3.968 & 0.474 & 0.356 \\
\hline 62.1 & 17 & 3 & Nannofossil chalk ooze & 159.75 & 1.69 & 60.4 & 1.595 & 2697 & 71.989 & 0.258 & 4.112 & 0.183 & 3.990 & 0.478 & 0.329 \\
\hline 62.1 & 17 & 4 & Nannofossil chalk ooze & 161.25 & 1.71 & 59.6 & 1.664 & 2837 & 69.310 & 0.279 & 4.178 & 0.571 & 3.797 & 0.435 & 0.579 \\
\hline 62.1 & 17 & 5 & Nannofossil chalk ooze & 162.75 & 1.71 & 59.2 & 1.626 & 2785 & 70.473 & 0.291 & 4.219 & 0.253 & 4.050 & 0.471 & 0.385 \\
\hline
\end{tabular}

*NOTES: Depth $=$ depth below sea floor, meters $; \rho_{\mathrm{B}}=$ saturated bulk density, $\mathrm{gm} / \mathrm{cm}^{3} ; \phi=$ porosity, fraction of total volume; $\mathrm{V}_{\mathrm{P}}=$ velocity, compressional wave, $\mathrm{km} / \mathrm{sec} ; \mathrm{Imped}-$ ance $=\mathrm{gm} / \mathrm{cm}^{2} \sec \times 10^{2} ; \mathrm{K}_{\mathrm{G}}=$ weighted mean bulk modulus, dynes $/ \mathrm{cm}^{2} \times 10^{10} ; \mathrm{K}_{\mathrm{S}}=$ skeletal bulk modulus, dynes $/ \mathrm{cm}^{2} \times 10^{10} ; \mathrm{K}=$ bulk modulus sediment, dynes $/ \mathrm{cm}{ }^{2} \times$ $10^{10} ; \mu=$ rigidity, dynes $/ \mathrm{cm}^{2} \times 10^{10} ; \lambda=$ Lame's constant, dynes $/ \mathrm{cm}^{2} \times 10^{10} ; \theta=$ Poisson's ratio; $\mathrm{v}_{\mathrm{S}}=$ velocity of shear wave in $\mathrm{km} / \mathrm{sec}$. 


\begin{tabular}{|c|c|c|c|c|c|c|c|c|c|c|c|c|c|c|c|}
\hline \multicolumn{3}{|c|}{ Identification } & \multirow[b]{2}{*}{ Lithology } & \multirow[b]{2}{*}{ Depth* } & \multirow[b]{2}{*}{$\rho_{\mathrm{B}}$} & \multirow[b]{2}{*}{$\phi$} & \multirow[b]{2}{*}{$V_{P}$} & \multirow{2}{*}{$\begin{array}{l}\text { Acoustic } \\
\text { Impedance }\end{array}$} & \multirow[b]{2}{*}{$\mathrm{K}_{\mathrm{G}}$} & \multirow[b]{2}{*}{$\mathrm{K}_{\mathrm{S}}$} & \multirow[b]{2}{*}{ K } & \multirow[b]{2}{*}{$\mu$} & \multirow[b]{2}{*}{ l } & \multirow[b]{2}{*}{$\theta$} & \multirow[b]{2}{*}{$\mathrm{v}_{\mathrm{S}}$} \\
\hline Hole & Core & Section & & & & & & & & & & & & & \\
\hline 62.1 & 17 & 6 & Nannofossil chalk ooze & 164.25 & 1.72 & 58.6 & 1.575 & 2711 & 67.020 & 0.306 & 4.257 & 0.037 & 4.233 & 0.496 & 0.147 \\
\hline 62.1 & 18 & 1 & Nannofossil chalk ooze & 165.75 & 1.77 & 55.6 & 1.669 & 2959 & 68.711 & 0.406 & 4.553 & 0.510 & 4.213 & 0.446 & 0.536 \\
\hline 62.1 & 18 & 2 & Nannofossil chalk ooze & 167.25 & 1.77 & 56.0 & 1.706 & 3012 & 69.804 & 0.392 & 4.517 & 0.558 & 4.145 & 0.441 & 0.562 \\
\hline 62.1 & 18 & 3 & Nannofossil chalk ooze & 168.75 & 1.72 & 58.9 & 1.669 & 2865 & 70.826 & 0.297 & 4.239 & 0.466 & 3.928 & 0.447 & 0.521 \\
\hline 62.1 & 18 & 4 & Nannofossil chalk ooze & 170.25 & 1.76 & 56.6 & 1.683 & 2956 & 70.649 & 0.370 & 4.457 & 0.495 & 4.127 & 0.446 & 0.531 \\
\hline 62.1 & 18 & 5 & Nannofossil chalk ooze & 171.75 & 1.74 & 57.8 & 1.711 & 2969 & 68.676 & 0.330 & 4.335 & 0.644 & 3.905 & 0.429 & 0.609 \\
\hline 62.1 & 18 & 6 & Nannofossil chalk ooze & 173.25 & 1.77 & 55.9 & 1.646 & 2908 & 68.993 & 0.394 & 4.521 & 0.399 & 4.255 & 0.457 & 0.475 \\
\hline 62.1 & 19 & 2 & Nannofossil chalk ooze & 176.25 & 1.68 & 61.1 & 1.700 & 2857 & 71.037 & 0.243 & 4.060 & 0.726 & 3.576 & 0.416 & 0.657 \\
\hline 62.1 & 19 & 3 & Nannofossil chalk ooze & 177.75 & 1.77 & 55.5 & 1.716 & 3044 & 71.530 & 0.410 & 4.569 & 0.532 & 4.214 & 0.444 & 0.548 \\
\hline 62.1 & 19 & 4 & Nannofossil chalk ooze & 179.25 & 1.76 & 56.3 & 1.682 & 2962 & 70.262 & 0.381 & 4.488 & 0.518 & 4.143 & 0.444 & 0.543 \\
\hline 62.1 & 19 & 5 & Nannofossil chalk ooze & 180.75 & 1.71 & 59.6 & 1.650 & 2814 & 70.121 & 0.280 & 4.182 & 0.462 & 3.874 & 0.447 & 0.520 \\
\hline 62.1 & 19 & 6 & Nannofossil chalk ooze & 182.25 & 1.77 & 56.0 & 1.625 & 2868 & 59.549 & 0.389 & 4.483 & 0.163 & 4.374 & 0.482 & 0.304 \\
\hline 62.1 & 20 & 1 & Nannofossil chalk ooze & 186.75 & 1.72 & 59.0 & 1.661 & 2849 & 69.698 & 0.295 & 4.231 & 0.500 & 3.897 & 0.443 & 0.540 \\
\hline 62.1 & 20 & 2 & Nannofossil chalk ooze & 188.25 & 1.74 & 57.8 & 1.658 & 2878 & 65.540 & 0.332 & 4.335 & 0.376 & 4.084 & 0.458 & 0.465 \\
\hline 62.1 & 20 & 3 & Nannofossil chalk ooze & 189.75 & 1.74 & 57.4 & 1.638 & 2853 & 70.508 & 0.343 & 4.378 & 0.231 & 4.224 & 0.474 & 0.364 \\
\hline 62.1 & 20 & 4 & Nannofossil chalk ooze & 191.25 & 1.77 & 56.1 & 1.663 & 2935 & 69.205 & 0.389 & 4.506 & 0.334 & 4.284 & 0.464 & 0.435 \\
\hline 62.1 & 20 & 5 & Nannofossil chalk ooze & 192.75 & 1.74 & 57.3 & 1.646 & 2870 & 70.473 & 0.346 & 4.387 & 0.353 & 4.152 & 0.461 & 0.450 \\
\hline 62.1 & 20 & 6 & Nannofossil chalk ooze & 194.25 & 1.75 & 56.9 & 1.536 & 2688 & 70.438 & 0.359 & 4.424 & 0.000 & 4.424 & 0.500 & 0.000 \\
\hline 62.1 & 21 & 1 & $\begin{array}{l}\text { Nannofossil chalk ooze } \\
\text { and chalk }\end{array}$ & 195.75 & 1.72 & 58.7 & 1.572 & 2705 & 68.993 & 0.305 & 4.259 & 0.061 & 4.218 & 0.493 & 0.188 \\
\hline 62.1 & 21 & 2 & $\begin{array}{l}\text { Nannofossil chalk ooze } \\
\text { and chalk }\end{array}$ & 197.25 & 1.78 & 55.3 & 1.682 & 3010 & 70.931 & 0.417 & 4.589 & 0.358 & 4.350 & 0.462 & 0.449 \\
\hline 62.1 & 21 & 3 & $\begin{array}{l}\text { Nannofossil chalk ooze } \\
\text { and chalk }\end{array}$ & 198.75 & 1.78 & 55.0 & 1.595 & 2843 & 69.205 & 0.429 & 4.616 & 0.021 & 4.602 & 0.498 & 0.109 \\
\hline 62.1 & 21 & 4 & $\begin{array}{l}\text { Nannofossil chalk ooze } \\
\text { and chalk }\end{array}$ & 200.25 & 1.80 & 54.1 & 1.594 & 2865 & 67.055 & 0.467 & 4.710 & 0.000 & 4.710 & 0.500 & 0.000 \\
\hline
\end{tabular}




\begin{tabular}{|c|c|c|c|c|c|c|c|c|c|c|c|c|c|c|c|}
\hline 62.1 & 21 & 5 & $\begin{array}{l}\text { Nannofossil chalk ooze } \\
\text { and chalk }\end{array}$ & 201.75 & 1.80 & 54.0 & 1.692 & 3027 & 70.614 & 0.473 & 4.734 & 0.384 & 4.478 & 0.461 & 0.462 \\
\hline 62.1 & 21 & 6 & $\begin{array}{l}\text { Nannofossil chalk ooze } \\
\text { and chalk }\end{array}$ & 203.25 & 1.80 & 53.7 & 1.580 & 2850 & 63.954 & 0.483 & 4.743 & 0.000 & 4.743 & 0.500 & 0.000 \\
\hline 62.1 & 22 & 1 & $\begin{array}{l}\text { Nannofossil chalk ooze } \\
\text { and chalk }\end{array}$ & 207.75 & 1.76 & 56.3 & 1.523 & 2682 & 70.191 & 0.380 & 4.485 & 0.000 & 4.485 & 0.500 & 0.000 \\
\hline 62.1 & 22 & 2 & $\begin{array}{l}\text { Nannofossil chalk ooze } \\
\text { and chalk }\end{array}$ & 209.25 & 1.77 & 56.0 & 1.632 & 2882 & 70.332 & 0.391 & 4.515 & 0.159 & 4.409 & 0.483 & 0.300 \\
\hline 62.1 & 22 & 3 & $\begin{array}{l}\text { Nannofossil chalk ooze } \\
\text { and chalk }\end{array}$ & 210.75 & 1.81 & 53.5 & 1.633 & 2953 & 69.346 & 0.494 & 4.785 & 0.522 & 4.437 & 0.447 & 0.537 \\
\hline 62.1 & 22 & 4 & $\begin{array}{l}\text { Nannofossil chalk ooze } \\
\text { and chalk }\end{array}$ & 212.25 & 1.78 & 55.2 & 1.585 & 2822 & 69.592 & 0.422 & 4.600 & 0.084 & 4.544 & 0.491 & 0.217 \\
\hline 62.1 & 22 & 5 & $\begin{array}{l}\text { Nannofossil chalk ooze } \\
\text { and chalk }\end{array}$ & 213.75 & 1.82 & 52.7 & 1.688 & 3076 & 70.156 & 0.533 & 4.883 & 0.283 & 4.694 & 0.472 & 0.394 \\
\hline 62.1 & 22 & 6 & $\begin{array}{l}\text { Nannofossil chalk ooze } \\
\text { and chalk }\end{array}$ & 215.25 & 1.74 & 57.4 & 1.539 & 2680 & 69.909 & 0.343 & 4.377 & 0.000 & 4.377 & 0.500 & 0.000 \\
\hline 62.1 & 23 & 1 & $\begin{array}{l}\text { Nannofossil chalk ooze } \\
\text { and chalk }\end{array}$ & 216.75 & 1.77 & 55.8 & 1.541 & 2727 & 68.676 & 0.398 & 4.530 & 0.000 & 4.530 & 0.500 & 0.000 \\
\hline 62.1 & 23 & 2 & $\begin{array}{l}\text { Nannofossil chalk ooze } \\
\text { and chalk }\end{array}$ & 218.25 & 1.79 & 54.5 & 1.646 & 2949 & 64.201 & 0.451 & 4.662 & 0.257 & 4.491 & 0.473 & 0.378 \\
\hline 62.1 & 23 & 3 & $\begin{array}{l}\text { Nannofossil chalk ooze } \\
\text { and chalk }\end{array}$ & 219.75 & 1.79 & 54.2 & 1.537 & 2759 & 58.950 & 0.460 & 4.669 & 0.000 & 4.669 & 0.500 & 0.000 \\
\hline 62.1 & 23 & 4 & $\begin{array}{l}\text { Nannofossil chalk ooze } \\
\text { and chalk }\end{array}$ & 221.25 & 1.80 & 53.9 & 1.546 & 2784 & 69.134 & 0.475 & 4.736 & 0.000 & 4.736 & 0.500 & 0.000 \\
\hline 62.1 & 23 & 5 & $\begin{array}{l}\text { Nannofossil chalk ooze } \\
\text { and chalk }\end{array}$ & 222.75 & 1.79 & 54.6 & 1.693 & 3030 & 62.227 & 0.447 & 4.646 & 0.474 & 4.330 & 0.451 & 0.514 \\
\hline 62.1 & 24 & 1 & $\begin{array}{l}\text { Nannofossil chalk and } \\
\text { chalk ooze }\end{array}$ & 225.75 & 1.82 & 53.0 & 1.592 & 2893 & 72.059 & 0.519 & 4.854 & 0.000 & 4.854 & 0.500 & 0.000 \\
\hline 62.1 & 24 & 2 & $\begin{array}{l}\text { Nannofossil chalk and } \\
\text { chalk ooze }\end{array}$ & 227.25 & 1.75 & 56.7 & 1.748 & 3067 & 70.826 & 0.365 & 4.443 & 0.747 & 3.945 & 0.420 & 0.653 \\
\hline
\end{tabular}

*NOTES: Depth $=$ depth below sea floor, meters $; \rho_{\mathrm{B}}=$ saturated bulk density, $\mathrm{gm} / \mathrm{cm}^{3} ; \phi=$ porosity, fraction of total volume; $\mathrm{V}_{\mathrm{P}}=$ velocity, compressional wave, $\mathrm{km} / \mathrm{sec} ; \mathrm{Imped}-$ ance $=\mathrm{gm} / \mathrm{cm}^{2} \sec \times 10^{2} ; \mathrm{K}_{\mathrm{G}}=$ weighted mean bulk modulus, dynes $/ \mathrm{cm}^{2} \times 10^{10} ; \mathrm{K}_{\mathrm{S}}=$ skeletal bulk modulus, dynes $/ \mathrm{cm}^{2} \times 10^{10} ; \mathrm{K}=$ bulk modulus sediment, dynes $/ \mathrm{cm}^{2} \times$ $10^{10} ; \mu=$ rigidity, dynes $/ \mathrm{cm}^{2} \times 10^{10} ; \lambda=$ Lame's constant, dynes $/ \mathrm{cm}^{2} \times 10^{10} ; \theta=$ Poisson's ratio; $\mathrm{V}_{\mathrm{S}}=$ velocity of shear wave in $\mathrm{km} / \mathrm{sec}$. 


\begin{tabular}{|c|c|c|c|c|c|c|c|c|c|c|c|c|c|c|c|}
\hline \multicolumn{3}{|c|}{ Identification } & \multirow[b]{2}{*}{ Lithology } & \multirow[b]{2}{*}{ Depth* } & \multirow[b]{2}{*}{$\rho_{\mathrm{B}}$} & \multirow[b]{2}{*}{$\phi$} & \multirow[b]{2}{*}{$\mathrm{V}_{\mathrm{P}}$} & \multirow{2}{*}{$\begin{array}{l}\text { Acoustic } \\
\text { Impedance }\end{array}$} & \multirow[b]{2}{*}{$\mathrm{K}_{\mathrm{G}}$} & \multirow[b]{2}{*}{$\mathrm{K}_{\mathrm{S}}$} & \multirow[b]{2}{*}{ K } & \multirow[b]{2}{*}{$\mu$} & \multirow[b]{2}{*}{$\lambda$} & \multirow[b]{2}{*}{$\theta$} & \multirow[b]{2}{*}{$\mathrm{v}_{\mathrm{S}}$} \\
\hline Hole & Core & Section & & & & & & & & & & & & & \\
\hline 62.1 & 24 & 3 & $\begin{array}{l}\text { Nannofossil chalk and } \\
\text { chalk ooze }\end{array}$ & 228.75 & 1.78 & 54.9 & 1.595 & 2846 & 69.416 & 0.433 & 4.628 & 0.000 & 4.628 & 0.500 & 0.000 \\
\hline 62.1 & 24 & 4 & $\begin{array}{l}\text { Nannofossil chalk and } \\
\text { chalk ooze }\end{array}$ & 230.25 & 1.78 & 54.9 & 1.636 & 2918 & 69.592 & 0.431 & 4.623 & 0.136 & 4.533 & 0.486 & 0.276 \\
\hline 62.1 & 24 & 5 & $\begin{array}{l}\text { Nannofossil chalk and } \\
\text { chalk ooze }\end{array}$ & 231.75 & 1.77 & 55.7 & 1.716 & 3039 & 62.051 & 0.403 & 4.528 & 0.561 & 4.153 & 0.441 & 0.563 \\
\hline 62.1 & 24 & 6 & $\begin{array}{l}\text { Nannofossil chalk and } \\
\text { chalk ooze }\end{array}$ & 233.25 & 1.79 & 54.8 & 1.576 & 2815 & 68.006 & 0.436 & 4.632 & 0.000 & 4.632 & 0.500 & 0.000 \\
\hline 62.1 & 25 & 1 & $\begin{array}{l}\text { Nannofossil chalk and } \\
\text { chalk ooze }\end{array}$ & 234.75 & 1.74 & 57.5 & 1.584 & 2757 & 69.592 & 0.339 & 4.364 & 0.105 & 4.294 & 0.488 & 0.246 \\
\hline 62.1 & 25 & 2 & $\begin{array}{l}\text { Nannofossil chalk and } \\
\text { chalk ooze }\end{array}$ & 236.25 & 1.44 & 75.2 & 1.687 & 2434 & 68.394 & 0.065 & 3.212 & 0.734 & 2.723 & 0.394 & 0.713 \\
\hline 62.1 & 25 & 3 & $\begin{array}{l}\text { Nannofossil chalk and } \\
\text { chalk ooze }\end{array}$ & 237.75 & 1.71 & 59.4 & 1.582 & 2704 & 68.958 & 0.285 & 4.195 & 0.139 & 4.103 & 0.484 & 0.285 \\
\hline 62.1 & 25 & 4 & $\begin{array}{l}\text { Nannofossil chalk and } \\
\text { chalk ooze }\end{array}$ & 239.25 & 1.74 & 57.4 & 1.586 & 2765 & 67.760 & 0.344 & 4.375 & 0.099 & 4.309 & 0.489 & 0.238 \\
\hline 62.1 & 25 & 5 & $\begin{array}{l}\text { Nannofossil chalk and } \\
\text { chalk ooze }\end{array}$ & 240.75 & 1.41 & 77.3 & 1.655 & 2327 & 68.465 & 0.053 & 3.117 & 0.632 & 2.695 & 0.405 & 0.670 \\
\hline 62.1 & 25 & 6 & $\begin{array}{l}\text { Nannofossil chalk and } \\
\text { chalk ooze }\end{array}$ & 242.25 & 1.73 & 58.0 & 1.574 & 2727 & & & & & & & \\
\hline 62.1 & 26 & 1 & $\begin{array}{l}\text { Nannofossil chalk and } \\
\text { chalk ooze }\end{array}$ & 245.75 & 1.75 & 56.9 & 1.656 & 2897 & 64.623 & 0.358 & 4.408 & 0.425 & 4.125 & 0.453 & 0.493 \\
\hline 62.1 & 26 & 2 & $\begin{array}{l}\text { Nannofossil chalk and } \\
\text { chalk ooze }\end{array}$ & 247.25 & 1.72 & 58.9 & 1.644 & 2821 & & & & & & & \\
\hline 62.1 & 26 & 3 & $\begin{array}{l}\text { Nannofossil chalk and } \\
\text { chalk ooze }\end{array}$ & 248.75 & 1.75 & 56.7 & 1.544 & 2709 & 67.584 & 0.366 & 4.439 & 0.000 & 4.439 & 0.500 & 0.000 \\
\hline 62.1 & 26 & 4 & $\begin{array}{l}\text { Nannofossil chalk and } \\
\text { chalk ooze }\end{array}$ & 250.25 & 1.77 & 56.1 & 1.601 & 2826 & 67.407 & 0.388 & 4.502 & 0.149 & 4.402 & 0.484 & 0.291 \\
\hline
\end{tabular}




\begin{tabular}{|c|c|c|c|c|c|c|c|c|c|c|c|c|c|c|c|}
\hline 62.1 & 26 & 5 & $\begin{array}{l}\text { Nannofossil chalk and } \\
\text { chalk ooze }\end{array}$ & 251.75 & 1.73 & 58.2 & 1.648 & 2849 & 70.508 & 0.319 & 4.305 & 0.336 & 4.081 & 0.462 & 0.441 \\
\hline 62.1 & 26 & 6 & $\begin{array}{l}\text { Nannofossil chalk and } \\
\text { chalk ooze }\end{array}$ & 253.25 & 1.69 & 60.5 & 1.549 & 2620 & 67.760 & 0.258 & 4.104 & 0.076 & 4.054 & 0.491 & 0.212 \\
\hline 62.1 & 27 & 2 & $\begin{array}{l}\text { Nannofossil chalk and } \\
\text { chalk ooze }\end{array}$ & 256.25 & 1.72 & 58.8 & 1.664 & 2859 & 68.006 & 0.301 & 4.244 & 0.385 & 3.987 & 0.456 & 0.473 \\
\hline 62.1 & 27 & 3 & $\begin{array}{l}\text { Nannofossil chalk and } \\
\text { chalk ooze }\end{array}$ & 257.75 & 1.74 & 57.7 & 1.587 & 2755 & 63.883 & 0.332 & 4.332 & 0.101 & 4.264 & 0.488 & 0.241 \\
\hline 62.1 & 27 & 4 & $\begin{array}{l}\text { Nannofossil chalk and } \\
\text { chalk ooze }\end{array}$ & 259.25 & 1.78 & 55.4 & 1.715 & 3045 & 68.711 & 0.414 & 4.576 & 0.541 & 4.215 & 0.443 & 0.552 \\
\hline 62.1 & 27 & 5 & $\begin{array}{l}\text { Nannofossil chalk and } \\
\text { chalk ooze }\end{array}$ & 260.75 & 1.77 & 55.6 & 1.621 & 2874 & 69.275 & 0.406 & 4.554 & 0.130 & 4.467 & 0.486 & 0.271 \\
\hline 62.1 & 28 & 1 & $\begin{array}{l}\text { Nannofossil chalk and } \\
\text { chalk ooze }\end{array}$ & 263.75 & 1.69 & 60.8 & 1.595 & 2687 & 67.971 & 0.250 & 4.077 & 0.247 & 3.912 & 0.470 & 0.383 \\
\hline 62.1 & 28 & 2 & $\begin{array}{l}\text { Nannofossil chalk and } \\
\text { chalk ooze }\end{array}$ & 265.25 & 1.72 & 58.9 & 1.683 & 2890 & 68.288 & 0.298 & 4.237 & 0.566 & 3.860 & 0.436 & 0.574 \\
\hline 62.1 & 28 & 3 & $\begin{array}{l}\text { Nannofossil chalk and } \\
\text { chalk ooze }\end{array}$ & 266.75 & 1.75 & 56.7 & 1.610 & 2822 & 66.491 & 0.365 & 4.433 & 0.096 & 4.369 & 0.489 & 0.234 \\
\hline 62.1 & 28 & 4 & $\begin{array}{l}\text { Nannofossil chalk and } \\
\text { chalk ooze }\end{array}$ & 268.25 & 1.70 & 59.9 & 1.602 & 2725 & 63.883 & 0.273 & 4.147 & 0.184 & 4.024 & 0.478 & 0.329 \\
\hline 62.1 & 28 & 5 & $\begin{array}{l}\text { Nannofossil chalk and } \\
\text { chalk ooze }\end{array}$ & 269.75 & 1.67 & 61.6 & 1.693 & 2829 & 66.033 & 0.231 & 4.008 & 0.628 & 3.589 & 0.426 & 0.613 \\
\hline 62.1 & 28 & 6 & $\begin{array}{l}\text { Nannofossil chalk and } \\
\text { chalk ooze }\end{array}$ & 271.25 & 1.69 & 60.8 & 1.609 & 2711 & 55.990 & 0.249 & 4.052 & 0.278 & 3.867 & 0.467 & 0.406 \\
\hline 62.1 & 29 & 1 & $\begin{array}{l}\text { Nannofossil chalk and } \\
\text { chalk ooze }\end{array}$ & 271.75 & 1.69 & 60.3 & 1.640 & 2778 & 70.121 & 0.262 & 4.123 & 0.472 & 3.808 & 0.445 & 0.528 \\
\hline 62.1 & 29 & 2 & $\begin{array}{l}\text { Nannofossil chalk and } \\
\text { chalk ooze }\end{array}$ & 273.25 & 1.69 & 60.5 & 1.761 & 2974 & 68.147 & 0.256 & 4.098 & 1.257 & 3.260 & 0.361 & 0.863 \\
\hline 62.1 & 29 & 3 & $\begin{array}{l}\text { Nannofossil chalk and } \\
\text { chalk ooze }\end{array}$ & 274.75 & 1.71 & 59.3 & 1.673 & 2860 & 67.478 & 0.287 & 4.201 & 0.512 & 3.860 & 0.442 & 0.547 \\
\hline
\end{tabular}

*NOTES: Depth $=$ depth below sea floor, meters $; \rho_{\mathrm{B}}=$ saturated bulk density, $\mathrm{gm} / \mathrm{cm}^{3} ; \phi=$ porosity, fraction of total volume; $\mathrm{V}_{\mathrm{P}}=$ velocity, compressional wave, $\mathrm{km} / \mathrm{sec} ; \mathrm{Imped}-$ $=\quad$ ance $=\mathrm{gm} / \mathrm{cm}^{2} \sec \times 10^{2} ; \mathrm{K}_{\mathrm{G}}=$ weighted mean bulk modulus, dynes $/ \mathrm{cm}^{2} \times 10^{10} ; \mathrm{K}_{\mathrm{S}}=$ skeletal bulk modulus, dynes $/ \mathrm{cm}^{2} \times 10^{10} ; \mathrm{K}=$ bulk modulus sediment, dynes $/ \mathrm{cm}^{2} \times$ $\vec{u}$ $10^{10} ; \mu=$ rigidity, dynes $/ \mathrm{cm}^{2} \times 10^{10} ; \lambda=$ Lame's constant, dynes $/ \mathrm{cm}^{2} \times 10^{10} ; \theta=$ Poisson's ratio; $\mathrm{V}_{\mathrm{S}}=$ velocity of shear wave in $\mathrm{km} / \mathrm{sec}$. 


\begin{tabular}{|c|c|c|c|c|c|c|c|c|c|c|c|c|c|c|c|}
\hline \multicolumn{3}{|c|}{ Identification } & \multirow[b]{2}{*}{ Lithology } & \multirow[b]{2}{*}{ Depth* } & \multirow[b]{2}{*}{$\rho_{\mathrm{B}}$} & \multirow[b]{2}{*}{$\phi$} & \multirow[b]{2}{*}{$\mathrm{V}_{\mathrm{P}}$} & \multirow{2}{*}{$\begin{array}{l}\text { Acoustic } \\
\text { Impedance }\end{array}$} & \multirow[b]{2}{*}{$\mathrm{K}_{\mathrm{G}}$} & \multirow[b]{2}{*}{$\mathrm{K}_{\mathrm{S}}$} & \multirow[b]{2}{*}{ K } & & \multirow[b]{2}{*}{$\lambda$} & \multirow[b]{2}{*}{$\theta$} & \multirow[b]{2}{*}{$\mathrm{v}_{\mathrm{S}}$} \\
\hline Hole & Core & Section & & & & & & & & & & & & & \\
\hline 62.1 & 29 & 4 & $\begin{array}{l}\text { Nannofossil chalk and } \\
\text { chalk ooze }\end{array}$ & 276.25 & 1.68 & 60.9 & 1.645 & 2771 & 67.443 & 0.248 & 4.071 & 0.512 & 3.730 & 0.440 & 0.551 \\
\hline 62.1 & 29 & 5 & $\begin{array}{l}\text { Nannofossil chalk and } \\
\text { chalk ooze }\end{array}$ & 277.75 & 1.71 & 59.5 & 1.696 & 2894 & 68.147 & 0.281 & 4.182 & 0.632 & 3.761 & 0.428 & 0.608 \\
\hline 62.1 & 29 & 6 & $\begin{array}{l}\text { Nannofossil chalk and } \\
\text { chalk ooze }\end{array}$ & 279.25 & 1.70 & 59.9 & 1.655 & 2813 & 67.689 & 0.272 & 4.151 & 0.438 & 3.859 & 0.449 & 0.508 \\
\hline 62.1 & 30 & 1 & $\begin{array}{l}\text { Nannofossil chalk and } \\
\text { chalk ooze }\end{array}$ & 281.75 & 1.74 & 57.8 & 1.640 & 2845 & 68.535 & 0.329 & 4.333 & 0.306 & 4.130 & 0.466 & 0.420 \\
\hline 62.1 & 30 & 2 & $\begin{array}{l}\text { Nannofossil chalk and } \\
\text { chalk ooze }\end{array}$ & 283.25 & 1.72 & 58.6 & 1.744 & 3003 & 68.535 & 0.307 & 4.264 & 0.817 & 3.719 & 0.410 & 0.689 \\
\hline 62.1 & 30 & 3 & $\begin{array}{l}\text { Nannofossil chalk and } \\
\text { chalk ooze }\end{array}$ & 284.75 & 1.75 & 56.7 & 1.653 & 2899 & 68.570 & 0.365 & 4.439 & 0.388 & 4.180 & 0.458 & 0.470 \\
\hline 62.1 & 30 & 4 & $\begin{array}{l}\text { Nannofossil chalk and } \\
\text { chalk ooze }\end{array}$ & 286.25 & 1.74 & 57.4 & 1.636 & 2850 & 69.698 & 0.343 & 4.378 & 0.304 & 4.175 & 0.466 & 0.418 \\
\hline 62.1 & 30 & 5 & $\begin{array}{l}\text { Nannofossil chalk and } \\
\text { chalk ooze }\end{array}$ & 287.75 & 1.74 & 57.6 & 1.748 & 3039 & 69.099 & 0.337 & 4.358 & 1.000 & 3.692 & 0.394 & 0.758 \\
\hline 62.1 & 30 & 6 & $\begin{array}{l}\text { Nannofossil marl and } \\
\text { marl ooze }\end{array}$ & 289.25 & 1.71 & 59.2 & 1.620 & 2773 & 52.043 & 0.290 & 4.173 & 0.278 & 3.988 & 0.468 & 0.403 \\
\hline 62.1 & 31 & 1 & $\begin{array}{l}\text { Nannofossil chalk and } \\
\text { chalk ooze }\end{array}$ & 291.75 & 1.77 & 55.8 & 1.621 & 2868 & 68.570 & 0.397 & 4.528 & 0.195 & 4.398 & 0.479 & 0.332 \\
\hline 62.1 & 31 & 2 & $\begin{array}{l}\text { Nannofossil chalk and } \\
\text { chalk ooze }\end{array}$ & 293.25 & 1.75 & 56.7 & 1.792 & 3143 & 67.936 & 0.365 & 4.438 & 1.233 & 3.616 & 0.373 & 0.838 \\
\hline 62.1 & 31 & 3 & $\begin{array}{l}\text { Nannofossil chalk and } \\
\text { chalk ooze }\end{array}$ & 294.75 & 1.78 & 55.1 & 1.643 & 2926 & 68.359 & 0.425 & 4.604 & 0.237 & 4.446 & 0.475 & 0.365 \\
\hline 62.1 & 31 & 4 & $\begin{array}{l}\text { Nannofossil chalk and } \\
\text { chalk ooze }\end{array}$ & 296.25 & 1.79 & 54.6 & 1.614 & 2889 & 68.006 & 0.447 & 4.662 & 0.110 & 4.588 & 0.488 & 0.248 \\
\hline 62.1 & 31 & 5 & $\begin{array}{l}\text { Nannofossil chalk and } \\
\text { chalk ooze }\end{array}$ & 297.75 & 1.70 & 59.9 & 1.727 & 2937 & 68.746 & 0.272 & 4.155 & 0.773 & 3.640 & 0.412 & 0.674 \\
\hline
\end{tabular}




\begin{tabular}{|c|c|c|c|c|c|c|c|c|c|c|c|c|c|c|c|}
\hline 62.1 & 31 & 6 & $\begin{array}{l}\text { Nannofossil chalk and } \\
\text { chalk ooze }\end{array}$ & 299.25 & 1.78 & 55.2 & 1.610 & 2865 & 69.240 & 0.424 & 4.602 & 0.065 & 4.559 & 0.493 & 0.191 \\
\hline 62.1 & 32 & 1 & Nannofossil chalk & 301.75 & 1.73 & 58.4 & 1.705 & 2941 & 69.169 & 0.312 & 4.282 & 0.809 & 3.743 & 0.411 & 0.685 \\
\hline 62.1 & 32 & 2 & Nannofossil chalk & 303.25 & 1.75 & 56.8 & 1.775 & 3110 & 69.627 & 0.363 & 4.434 & 0.937 & 3.809 & 0.401 & 0.731 \\
\hline 62.1 & 32 & 3 & Nannofossil chalk & 304.75 & 1.74 & 57.6 & 1.705 & 2963 & 70.473 & 0.336 & 4.357 & 0.720 & 3.877 & 0.422 & 0.644 \\
\hline 62.1 & 32 & 4 & Nannofossil chalk & 306.25 & 1.73 & 58.1 & 1.761 & 3048 & 63.883 & 0.323 & 4.304 & 1.201 & 3.503 & 0.372 & 0.833 \\
\hline 62.1 & 32 & 5 & Nannofossil chalk & 307.75 & 1.73 & 58.2 & 1.816 & 3144 & 69.169 & 0.322 & 4.311 & 1.109 & 3.572 & 0.382 & 0.801 \\
\hline 62.1 & 32 & 6 & Nannofossil chalk & 309.25 & 1.73 & 58.1 & 1.788 & 3093 & 68.218 & 0.321 & 4.309 & 1.147 & 3.544 & 0.378 & 0.814 \\
\hline 62.1 & 33 & 2 & Nannofossil chalk & 312.25 & 1.75 & 56.9 & 1.809 & 3166 & 67.161 & 0.358 & 4.414 & 0.985 & 3.758 & 0.396 & 0.750 \\
\hline 62.1 & 33 & 3 & Nannofossil chalk & 313.75 & 1.77 & 55.9 & 1.678 & 2966 & 66.174 & 0.393 & 4.512 & 0.446 & 4.215 & 0.452 & 0.502 \\
\hline 62.1 & 34 & 1 & Nannofossil chalk & 320.75 & 1.73 & 57.9 & 1.818 & 3152 & 66.350 & 0.327 & 4.322 & 1.195 & 3.525 & 0.373 & 0.830 \\
\hline 62.1 & 34 & 2 & Nannofossil chalk & 322.25 & 1.81 & 53.6 & 1.805 & 3260 & 67.689 & 0.489 & 4.768 & 0.872 & 4.187 & 0.414 & 0.695 \\
\hline 62.1 & 34 & 3 & Nannofossil chalk & 323.75 & 1.79 & 54.5 & 1.818 & 3256 & 65.046 & 0.448 & 4.656 & 1.095 & 3.926 & 0.391 & 0.782 \\
\hline 62.1 & 34 & 4 & Nannofossil chalk & 325.25 & 1.80 & 54.2 & 1.791 & 3219 & 66.844 & 0.464 & 4.701 & 1.207 & 3.896 & 0.382 & 0.820 \\
\hline 62.1 & 34 & 5 & Nannofossil chalk & 326.75 & 1.84 & 51.9 & 1.926 & 3534 & 67.865 & 0.572 & 4.970 & 1.805 & 3.767 & 0.338 & 0.992 \\
\hline 62.1 & 34 & 6 & Nannofossil chalk & 328.25 & 1.83 & 52.0 & 1.829 & 3353 & 68.324 & 0.568 & 4.962 & 1.132 & 4.207 & 0.394 & 0.786 \\
\hline 62.1 & 35 & 1 & Nannofossil chalk & 327.75 & 1.71 & 59.2 & 1.591 & 2722 & 65.011 & 0.289 & 4.201 & 0.135 & 4.111 & 0.484 & 0.280 \\
\hline 62.1 & 35 & 2 & Nannofossil chalk & 329.25 & 1.71 & 59.1 & 1.813 & 3101 & 67.055 & 0.292 & 4.215 & 1.165 & 3.438 & 0.373 & 0.825 \\
\hline 62.1 & 35 & 3 & Nannofossil chalk & 330.75 & 1.74 & 57.5 & 1.757 & 3059 & 67.513 & 0.340 & 4.363 & 0.921 & 3.749 & 0.401 & 0.727 \\
\hline 62.1 & 35 & 4 & Nannofossil chalk & 332.25 & 1.75 & 57.2 & 1.807 & 3155 & 67.161 & 0.350 & 4.392 & 0.982 & 3.738 & 0.396 & 0.750 \\
\hline 62.1 & 35 & 5 & Nannofossil chalk & 333.75 & 1.73 & 58.3 & 1.771 & 3057 & 65.892 & 0.314 & 4.281 & 0.849 & 3.715 & 0.407 & 0.701 \\
\hline
\end{tabular}

Table 3. Site 63

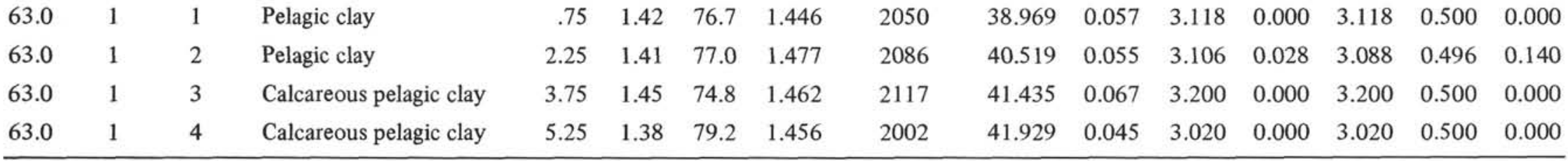

${ }^{*}$ NOTES: Depth $=$ depth below sea floor, meters $; \rho_{\mathrm{B}}=$ saturated bulk density, $\mathrm{gm} / \mathrm{cm}^{3} ; \phi=$ porosity, fraction of total volume; $\mathrm{V}_{\mathrm{P}}=$ velocity, compressional wave, $\mathrm{km} / \mathrm{sec} ; \mathrm{Imped}$ ance $=\mathrm{gm} / \mathrm{cm}^{2} \sec \times 10^{2} ; \mathrm{K}_{\mathrm{G}}=$ weighted mean bulk modulus, dynes $/ \mathrm{cm}^{2} \times 10^{10} ; \mathrm{K}_{\mathrm{S}}=$ skeletal bulk modulus, dynes $/ \mathrm{cm}^{2} \times 10^{10} ; \mathrm{K}=$ bulk modulus sediment, dynes $/ \mathrm{cm}^{2} \times$ $10^{10} ; \mu=$ rigidity, dynes $/ \mathrm{cm}^{2} \times 10^{10} ; \lambda=$ Lame's constant, dynes $/ \mathrm{cm}^{2} \times 10^{10} ; \theta=$ Poisson's ratio; $\mathrm{V}_{\mathrm{S}}=$ velocity of shear wave in $\mathrm{km} / \mathrm{sec}$. 


\begin{tabular}{|c|c|c|c|c|c|c|c|c|c|c|c|c|c|c|c|}
\hline \multicolumn{3}{|c|}{ Identification } & \multirow[b]{2}{*}{ Lithology } & \multirow[b]{2}{*}{ Depth* } & \multirow[b]{2}{*}{$\rho_{\mathrm{B}}$} & \multirow[b]{2}{*}{$\phi$} & \multirow[b]{2}{*}{$V_{P}$} & \multirow{2}{*}{$\begin{array}{l}\text { Acoustic } \\
\text { Impedance }\end{array}$} & \multirow[b]{2}{*}{$\mathrm{K}_{\mathrm{G}}$} & \multirow[b]{2}{*}{$\mathrm{K}_{\mathrm{S}}$} & \multirow[b]{2}{*}{ K } & \multirow[b]{2}{*}{$\mu$} & \multirow[b]{2}{*}{$\lambda$} & & \multirow[b]{2}{*}{$\mathrm{v}_{\mathrm{S}}$} \\
\hline Hole & Core & Section & & & & & & & & & & & & & \\
\hline 63.0 & 2 & 2 & Nannofossil chalk ooze & 63.25 & 1.63 & 64.2 & 1.563 & 2543 & 61.311 & 0.181 & 3.812 & 0.203 & 3.677 & 0.474 & 0.353 \\
\hline 63.0 & 2 & 3 & Nannofossil chalk ooze & 64.75 & 1.69 & 60.8 & 1.554 & 2619 & 66.244 & 0.251 & 4.077 & 0.026 & 4.060 & 0.497 & 0.123 \\
\hline 63.0 & 2 & 4 & Nannofossil chalk ooze & 66.25 & 1.63 & 64.2 & 1.546 & 2516 & 65.751 & 0.182 & 3.822 & 0.101 & 3.754 & 0.487 & 0.250 \\
\hline 63.0 & 2 & 5 & Nannofossil chalk ooze & 67.75 & 1.67 & 61.4 & 1.550 & 2594 & 66.244 & 0.236 & 4.025 & 0.030 & 4.000 & 0.495 & 0.149 \\
\hline 63.0 & 2 & 6 & Nannofossil chalk ooze & 69.25 & 1.70 & 59.9 & 1.552 & 2636 & 59.196 & 0.271 & 4.130 & 0.030 & 4.106 & 0.496 & 0.144 \\
\hline 63.0 & 3 & 2 & Nannofossil chalk ooze & 139.25 & 1.72 & 58.9 & 1.576 & 2706 & 60.254 & 0.299 & 4.223 & 0.030 & 4.202 & 0.496 & 0.135 \\
\hline 63.0 & 3 & 3 & Nannofossil chalk ooze & 140.75 & 1.73 & 58.1 & 1.615 & 2794 & 65.575 & 0.320 & 4.301 & 0.230 & 4.147 & 0.474 & 0.365 \\
\hline 63.0 & 3 & 4 & Nannofossil marl ooze & 142.25 & 1.71 & 59.3 & 1.720 & 2943 & 57.646 & 0.288 & 4.182 & 1.020 & 3.502 & 0.387 & 0.773 \\
\hline 63.0 & 4 & 1 & Nannofossil chalk ooze & 230.75 & 1.80 & 54.3 & 1.777 & 3190 & 64.623 & 0.460 & 4.684 & 0.950 & 4.047 & 0.405 & 0.730 \\
\hline 63.0 & 4 & 2 & Nannofossil chalk ooze & 232.25 & 1.88 & 49.4 & 1.952 & 3664 & 65.117 & 0.723 & 5.299 & 2.180 & 3.841 & 0.319 & 1.079 \\
\hline 63.0 & 5 & 1 & Nannofossil chalk & 252.75 & 1.81 & 53.3 & 1.524 & 2760 & 64.694 & 0.501 & 4.789 & 0.000 & 4.789 & 0.500 & 0.000 \\
\hline 63.0 & 5 & 2 & Nannofossil chalk & 354.25 & 1.91 & 47.5 & 2.110 & 4027 & 66.103 & 0.865 & 5.596 & 4.125 & 2.846 & 0.204 & 1.470 \\
\hline 63.0 & 6 & 1 & Nannofossil chalk & 458.75 & 1.94 & 45.7 & 1.692 & 3281 & 68.923 & 1.023 & 5.917 & 0.000 & 5.917 & 0.500 & 0.000 \\
\hline 63.0 & 6 & 2 & Nannofossil chalk & 460.25 & 1.90 & 47.9 & & 0 & 69.627 & 0.834 & 5.549 & 0.022 & 5.535 & 0.498 & 0.106 \\
\hline 63.0 & 6 & 3 & Nannofossil chalk & 461.75 & 1.96 & 44.6 & 1.640 & 3212 & 69.416 & 1.138 & 6.135 & 0.000 & 6.135 & 0.500 & 0.000 \\
\hline 63.0 & 6 & 4 & Nannofossil chalk & 463.25 & 1.95 & 45.3 & 1.737 & 3382 & 70.861 & 1.066 & 6.007 & 0.000 & 6.007 & 0.500 & 0.000 \\
\hline 63.0 & 6 & 5 & Nannofossil chalk & 464.75 & 1.97 & 43.6 & 1.597 & 3152 & 69.416 & 1.240 & 6.320 & 0.000 & 6.320 & 0.500 & 0.000 \\
\hline 63.0 & 6 & 6 & Nannofossil chalk & 466.25 & 1.96 & 44.5 & 1.736 & 3401 & 72.165 & 1.139 & 6.150 & 0.000 & 6.150 & 0.500 & 0.000 \\
\hline 63.0 & 7 & 1 & Nannofossil chalk & 534.75 & 1.97 & 44.0 & 1.778 & 3499 & 68.429 & 1.200 & 6.243 & 0.138 & 6.151 & 0.489 & 0.264 \\
\hline 63.0 & 7 & 2 & Nannofossil chalk & 536.25 & 1.97 & 44.2 & 1.776 & 3490 & 69.134 & 1.179 & 6.208 & 0.399 & 5.942 & 0.469 & 0.451 \\
\hline 63.0 & 7 & 3 & Nannofossil chalk & 537.75 & 2.01 & 41.7 & 1.768 & 3548 & 68.112 & 1.484 & 6.734 & 0.000 & 6.734 & 0.500 & 0.000 \\
\hline 63.0 & 7 & 4 & Nannofossil chalk & 539.25 & 2.01 & 41.3 & 1.719 & 3461 & 68.535 & 1.542 & 6.835 & 0.000 & 6.835 & 0.500 & 0.000 \\
\hline 63.0 & 7 & 5 & Nannofossil chalk & 540.75 & 2.04 & 39.9 & 1.791 & 3648 & 68.711 & 1.750 & 7.177 & 0.000 & 7.177 & 0.500 & 0.000 \\
\hline 63.0 & 7 & 6 & Nannofossil chalk & 542.25 & 2.05 & 39.3 & 1.701 & 3483 & 70.826 & 1.865 & 7.377 & 0.000 & 7.377 & 0.500 & 0.000 \\
\hline 63.0 & 8 & 2 & Nannofossil chalk & 545.25 & 1.99 & 43.8 & 1.786 & 3562 & 69.099 & 1.219 & 6.279 & 0.115 & 6.203 & 0.491 & 0.240 \\
\hline 63.0 & 8 & 3 & Nannofossil chalk & 546.75 & 2.02 & 42.4 & 1.854 & 3743 & 67.654 & 1.396 & 6.582 & 0.628 & 6.163 & 0.454 & 0.558 \\
\hline
\end{tabular}




\begin{tabular}{|c|c|c|c|c|c|c|c|c|c|c|c|c|c|c|c|}
\hline 63.0 & 9 & 1 & Nannofossil chalk & 553.75 & 2.08 & 38.8 & 1.872 & 3896 & 69.874 & 1.951 & 7.505 & 0.379 & 7.252 & 0.475 & 0.427 \\
\hline 63.0 & 9 & 2 & Nannofossil chalk & 555.25 & 2.11 & 38.5 & 1.872 & 3952 & 69.980 & 2.010 & 7.598 & 0.458 & 7.292 & 0.470 & 0.466 \\
\hline 63.0 & 9 & 3 & Nannofossil chalk & 556.75 & 2.16 & 35.9 & 1.917 & 4133 & 70.050 & 2.554 & 8.416 & 0.149 & 8.316 & 0.491 & 0.263 \\
\hline 63.0 & 9 & 4 & Nannofossil chalk & 558.25 & 2.17 & 35.3 & 2.103 & 4555 & 69.099 & 2.696 & 8.610 & 2.415 & 7.000 & 0.372 & 1.056 \\
\hline 63.1 & 3 & 2 & Calcareous pelagic clay & 24.25 & 1.40 & 77.6 & 1.416 & 1984 & 48.166 & 0.052 & 3.090 & 0.000 & 3.090 & 0.500 & 0.000 \\
\hline 63.1 & 5 & 1 & Nannofossil chalk ooze & 101.75 & 1.55 & 69.1 & 1.438 & 2222 & 59.690 & 0.115 & 3.509 & 0.000 & 3.509 & 0.500 & 0.000 \\
\hline 63.1 & 5 & 2 & Nannofossil chalk ooze & 103.25 & 1.61 & 65.2 & 1.435 & 2311 & 59.901 & 0.166 & 3.746 & 0.000 & 3.746 & 0.500 & 0.000 \\
\hline 63.1 & 5 & 3 & Nannofossil chalk ooze & 104.75 & 1.62 & 64.7 & 1.437 & 2327 & 62.791 & 0.173 & 3.783 & 0.000 & 3.783 & 0.500 & 0.000 \\
\hline 63.1 & 5 & 4 & Nannofossil marl ooze & 106.25 & 1.57 & 67.6 & 1.490 & 2339 & 53.664 & 0.132 & 3.587 & 0.000 & 3.587 & 0.500 & 0.000 \\
\hline 63.1 & 6 & 2 & Nannofossil marl ooze & 112.25 & 1.52 & 70.7 & 1.451 & 2204 & 50.527 & 0.099 & 3.413 & 0.000 & 3.413 & 0.500 & 0.000 \\
\hline 63.1 & 6 & 3 & Nannofossil marl ooze & 113.75 & 1.53 & 70.3 & 1.427 & 2176 & 53.734 & 0.103 & 3.438 & 0.000 & 3.438 & 0.500 & 0.000 \\
\hline 63.1 & 6 & 4 & Nannofossil marl ooze & 115.25 & 1.54 & 69.3 & 1.432 & 2209 & 56.483 & 0.113 & 3.495 & 0.000 & 3.495 & 0.500 & 0.000 \\
\hline 63.1 & 6 & 5 & Nannofossil marl ooze & 116.75 & 1.57 & 67.4 & 1.437 & 2261 & 53.382 & 0.135 & 3.602 & 0.000 & 3.602 & 0.500 & 0.000 \\
\hline 63.1 & 6 & 6 & Nannofossil marl ooze & 118.25 & 1.62 & 64.4 & 1.460 & 2371 & 57.012 & 0.178 & 3.792 & 0.000 & 3.792 & 0.500 & 0.000 \\
\hline 63.1 & 7 & 3 & Marl ooze & 122.75 & 1.53 & 69.9 & 1.461 & 2237 & 57.611 & 0.107 & 3.463 & 0.000 & 3.463 & 0.500 & 0.000 \\
\hline 63.1 & 7 & 4 & Marl ooze & 124.25 & 1.53 & 70.0 & 1.470 & 2249 & 53.523 & 0.106 & 3.453 & 0.000 & 3.453 & 0.500 & 0.000 \\
\hline 63.1 & 8 & 4 & Nannofossil chalk ooze & 134.25 & 1.38 & 79.2 & 1.453 & 1998 & 59.831 & 0.045 & 3.035 & 0.000 & 3.035 & 0.500 & 0.000 \\
\hline 63.1 & 8 & 5 & Nannofossil marl ooze & 135.75 & 1.42 & 76.8 & 1.473 & 2086 & 58.597 & 0.056 & 3.135 & 0.000 & 3.135 & 0.500 & 0.016 \\
\hline 63.1 & 9 & 3 & Nannofossil chalk & 141.75 & 1.66 & 62.1 & 1.534 & 2552 & 65.046 & 0.221 & 3.972 & 0.033 & 3.950 & 0.496 & 0.141 \\
\hline 63.1 & 9 & 4 & Nannofossil chalk & 143.25 & 1.75 & 56.9 & 1.457 & 2551 & 62.826 & 0.361 & 4.412 & 0.000 & 4.412 & 0.500 & 0.000 \\
\hline 63.1 & 9 & 5 & Nannofossil chalk & 144.75 & 1.70 & 60.1 & 1.472 & 2497 & 60.077 & 0.266 & 4.116 & 0.000 & 4.116 & 0.500 & 0.000 \\
\hline 63.1 & 9 & 6 & Nannofossil chalk & 146.25 & 1.73 & 58.3 & 1.522 & 2629 & 66.280 & 0.316 & 4.289 & 0.000 & 4.289 & 0.500 & 0.000 \\
\hline 63.1 & 10 & 1 & Nannofossil chalk & 148.75 & 1.71 & 59.6 & 1.496 & 2551 & 62.509 & 0.279 & 4.164 & 0.000 & 4.164 & 0.500 & 0.000 \\
\hline 63.1 & 10 & 2 & Nannofossil marl & 150.25 & 1.72 & 58.6 & 1.501 & 2586 & 56.483 & 0.308 & 4.239 & 0.000 & 4.239 & 0.500 & 0.000 \\
\hline 63.1 & 10 & 3 & Nannofossil chalk & 151.75 & 1.67 & 61.7 & 1.497 & 2498 & 62.967 & 0.229 & 3.995 & 0.000 & 3.995 & 0.500 & 0.000 \\
\hline 63.1 & 10 & 4 & Nannofossil chalk & 153.25 & 1.75 & 57.0 & 1.541 & 2696 & 60.677 & 0.356 & 4.394 & 0.000 & 4.394 & 0.500 & 0.000 \\
\hline
\end{tabular}

*NOTES: Depth $=$ depth below sea floor, meters $; \rho_{\mathrm{B}}=$ saturated bulk density, $\mathrm{gm} / \mathrm{cm}^{3} ; \phi=$ porosity, fraction of total volume; $\mathrm{V}_{\mathrm{P}}=$ velocity, compressional wave, $\mathrm{km} / \mathrm{sec} ; \mathrm{Imped}-$ ance $=\mathrm{gm} / \mathrm{cm}^{2} \sec \times 10^{2} ; \mathrm{K}_{\mathrm{G}}=$ weighted mean bulk modulus, dynes $/ \mathrm{cm}^{2} \times 10^{10} ; \mathrm{K}_{\mathrm{S}}=$ skeletal bulk modulus, dynes $/ \mathrm{cm}^{2} \times 10^{10} ; \mathrm{K}=$ bulk modulus sediment, dynes $/ \mathrm{cm}^{2} \mathrm{X}$ $10^{10} ; \mu=$ rigidity, dynes $/ \mathrm{cm}^{2} \times 10^{10} ; \lambda=$ Lame's constant, dynes $/ \mathrm{cm}^{2} \times 10^{10} ; \theta=$ Poisson's ratio; $\mathrm{V}_{\mathrm{S}}=$ velocity of shear wave in $\mathrm{km} / \mathrm{sec}$. 
TABLES 1 through 6 - Continued

\begin{tabular}{|c|c|c|c|c|c|c|c|c|c|c|c|c|c|c|c|}
\hline \multicolumn{3}{|c|}{ Identification } & \multirow[b]{2}{*}{ Lithology } & \multirow[b]{2}{*}{ Depth* } & \multirow[b]{2}{*}{$\rho_{\mathrm{B}}$} & \multirow[b]{2}{*}{$\phi$} & \multirow[b]{2}{*}{$\mathrm{V}_{\mathrm{P}}$} & \multirow{2}{*}{$\begin{array}{l}\text { Acoustic } \\
\text { Impedance }\end{array}$} & \multirow[b]{2}{*}{$\mathrm{K}_{\mathrm{G}}$} & \multirow[b]{2}{*}{$\mathrm{K}_{\mathrm{S}}$} & \multirow[b]{2}{*}{ K } & \multirow[b]{2}{*}{$\mu$} & \multirow[b]{2}{*}{$\lambda$} & \multirow[b]{2}{*}{$\theta$} & \multirow[b]{2}{*}{$\mathrm{v}_{\mathrm{S}}$} \\
\hline Hole & Core & Section & & & & & & & & & & & & & \\
\hline 63.1 & 10 & 5 & Nannofossil chalk & 154.75 & 1.76 & 56.6 & 1.474 & 2587 & 65.751 & 0.369 & 4.443 & 0.000 & 4.443 & 0.500 & 0.000 \\
\hline 63.1 & 11 & 1 & Nannofossil chalk & 155.75 & 1.70 & 59.6 & 1.486 & 2533 & 66.632 & 0.278 & 4.170 & 0.000 & 4.170 & 0.500 & 0.000 \\
\hline 63.1 & 11 & 2 & Nannofossil chalk & 157.25 & 1.66 & 62.5 & 1.470 & 2436 & 60.817 & 0.214 & 3.938 & 0.000 & 3.938 & 0.500 & 0.000 \\
\hline 63.1 & 11 & 3 & Nannofossil chalk & 158.75 & 1.73 & 58.2 & 1.496 & 2587 & 62.579 & 0.319 & 4.289 & 0.000 & 4.289 & 0.500 & 0.000 \\
\hline 63.1 & 11 & 4 & Nannofossil chalk & 160.25 & 1.76 & 56.3 & 1.504 & 2647 & 65.187 & 0.379 & 4.471 & 0.000 & 4.471 & 0.500 & 0.000 \\
\hline 63.1 & 11 & 5 & Nannofossil chalk & 161.75 & 1.76 & 56.1 & 1.541 & 2719 & 66.808 & 0.388 & 4.499 & 0.141 & 4.405 & 0.485 & 0.283 \\
\hline 63.1 & 11 & 6 & Nannofossil chalk & 163.25 & 1.79 & 54.4 & 1.615 & 2896 & 62.579 & 0.455 & 4.666 & 0.511 & 4.325 & 0.447 & 0.534 \\
\hline 63.1 & 12 & 1 & Nannofossil chalk & 165.75 & 1.76 & 56.1 & 1.726 & 3045 & & & & & & & \\
\hline 63.1 & 12 & 2 & Nannofossil chalk & 167.25 & 1.80 & 54.1 & 1.637 & 2941 & 66.632 & 0.465 & 4.704 & 0.111 & 4.630 & 0.488 & 0.248 \\
\hline 63.1 & 13 & 1 & Nannofossil chalk & 174.75 & 1.75 & 57.2 & 1.590 & 2775 & 64.659 & 0.348 & 4.380 & 0.369 & 4.134 & 0.459 & 0.460 \\
\hline 63.1 & 13 & 2 & Nannofossil chalk & 176.25 & 1.68 & 60.9 & 1.603 & 2699 & 63.742 & 0.248 & 4.063 & 0.543 & 3.701 & 0.436 & 0.568 \\
\hline 63.1 & 13 & 3 & Nannofossil chalk & 177.75 & 1.77 & 55.7 & 1.651 & 2924 & 65.187 & 0.403 & 4.537 & 0.768 & 4.025 & 0.420 & 0.658 \\
\hline 63.1 & 13 & 4 & Nannofossil chalk & 179.25 & 1.64 & 63.3 & 1.635 & 2685 & 63.179 & 0.345 & 4.367 & 0.853 & 3.798 & 0.408 & 0.699 \\
\hline 63.1 & 13 & 5 & Nannofossil chalk & 180.75 & 1.74 & 57.4 & 1.613 & 2812 & 59.126 & 0.197 & 3.871 & 0.589 & 3.479 & 0.428 & 0.599 \\
\hline 63.1 & 13 & 6 & Nannofossil marl & 182.25 & 1.62 & 64.7 & 1.576 & 2552 & 54.016 & 0.173 & 3.766 & 0.772 & 3.252 & 0.404 & 0.690 \\
\hline 63.1 & 14 & 1 & Nannofossil chalk & 184.75 & 1.69 & 60.8 & 1.535 & 2586 & 61.663 & 0.249 & 4.065 & 0.309 & 3.859 & 0.463 & 0.428 \\
\hline 63.1 & 14 & 2 & Nannofossil chalk & 186.25 & 1.78 & 55.4 & 1.534 & 2725 & 65.998 & 0.414 & 4.567 & 0.000 & 4.567 & 0.500 & 0.000 \\
\hline 63.1 & 14 & 3 & Nannofossil chalk & 187.75 & 1.72 & 58.6 & 1.681 & 2895 & 63.707 & 0.307 & 4.254 & 0.842 & 3.692 & 0.407 & 0.699 \\
\hline 63.1 & 14 & 4 & Nannofossil marl & 189.25 & 1.75 & 57.0 & 1.566 & 2738 & 58.174 & 0.356 & 4.387 & 0.264 & 4.212 & 0.471 & 0.388 \\
\hline 63.1 & 14 & 5 & Nannofossil chalk & 190.75 & 1.79 & 54.5 & 1.545 & 2767 & 67.020 & 0.449 & 4.662 & 0.000 & 4.662 & 0.500 & 0.000 \\
\hline 63.1 & 14 & 6 & Nannofossil chalk & 192.25 & 1.79 & 54.8 & 1.551 & 2769 & & & & & & & \\
\hline 63.2 & 1 & 1 & Calcareous pelagic clay & 11.75 & 1.32 & 82.5 & 1.379 & 1819 & & & & & & & \\
\hline 63.2 & 1 & 2 & Calcareous pelagic clay & 13.25 & 1.39 & 78.3 & 1.392 & 1935 & & & & & & & \\
\hline 63.2 & 1 & 3 & Calcareous pelagic clay & 14.75 & 1.37 & 79.4 & 1.364 & 1870 & 47.180 & 0.044 & 3.017 & 0.000 & 3.017 & 0.500 & 0.000 \\
\hline 63.2 & 1 & 4 & Calcareous pelagic clay & 16.25 & 1.32 & 82.3 & 1.379 & 1823 & 43.973 & 0.034 & 2.908 & 0.000 & 2.908 & 0.500 & 0.000 \\
\hline
\end{tabular}




\begin{tabular}{|c|c|c|c|c|c|c|c|c|c|c|c|c|c|c|c|}
\hline 63.2 & 2 & 1 & Calcareous pelagic clay & 20.75 & 1.36 & 80.1 & 1.330 & 1808 & 39.779 & 0.041 & 2.983 & 0.000 & 2.983 & 0.500 & 0.000 \\
\hline 63.2 & 2 & 2 & Calcareous pelagic clay & 22.25 & 1.32 & 82.5 & 1.374 & 1811 & 47.497 & 0.033 & 2.902 & 0.000 & 2.902 & 0.500 & 0.000 \\
\hline 63.2 & 2 & 3 & Calcareous pelagic clay & 23.75 & 1.30 & 83.8 & 1.416 & 1838 & 43.797 & 0.029 & 2.857 & 0.000 & 2.857 & 0.500 & 0.000 \\
\hline 63.2 & 2 & 4 & Calcareous pelagic clay & 25.25 & 1.30 & 83.9 & 1.364 & 1768 & 45.136 & 0.029 & 2.855 & 0.000 & 2.855 & 0.500 & 0.000 \\
\hline 63.2 & 3 & 1 & Calcareous pelagic clay & 30.75 & 1.35 & 80.5 & 1.434 & 1940 & 43.691 & 0.040 & 2.974 & 0.000 & 2.974 & 0.500 & 0.000 \\
\hline 63.2 & 3 & 2 & Calcareous pelagic clay & 32.25 & 1.39 & 78.3 & 1.387 & 1928 & 42.669 & 0.049 & 3.057 & 0.000 & 3.057 & 0.500 & 0.000 \\
\hline 63.2 & 3 & 3 & Nannofossil marl ooze & 33.75 & 1.46 & 74.2 & 1.406 & 2050 & 56.095 & 0.071 & 3.245 & 0.000 & 3.245 & 0.500 & 0.000 \\
\hline 63.2 & 3 & 4 & Nannofossil marl ooze & 35.25 & 1.55 & 68.7 & 1.405 & 2181 & 56.800 & 0.120 & 3.530 & 0.000 & 3.530 & 0.500 & 0.000 \\
\hline 64.0 & 1 & 3 & $\begin{array}{l}\text { Foraminiferal nanno- } \\
\text { fossil chalk ooze }\end{array}$ & 3.75 & 1.50 & 71.9 & 1.441 & 2159 & 66.103 & 0.089 & 3.369 & 0.000 & 3.369 & 0.500 & 0.000 \\
\hline 64.0 & 1 & 4 & $\begin{array}{l}\text { Foraminiferal nanno- } \\
\text { fossil chalk ooze }\end{array}$ & 5.25 & 1.51 & 71.3 & 1.445 & 2179 & 65.117 & 0.094 & 3.397 & 0.000 & 3.397 & 0.500 & 0.000 \\
\hline 64.0 & 1 & 5 & $\begin{array}{l}\text { Foraminiferal nanno- } \\
\text { fossil chalk ooze }\end{array}$ & 6.75 & 1.51 & 71.5 & 1.433 & 2156 & 66.808 & 0.092 & 3.391 & 0.000 & 3.391 & 0.500 & 0.000 \\
\hline 64.0 & 2 & 1 & $\begin{array}{l}\text { Foraminiferal nanno- } \\
\text { fossil chalk ooze }\end{array}$ & 99.75 & 1.61 & 65.5 & 1.455 & 2337 & 68.218 & 0.161 & 3.738 & 0.000 & 3.738 & 0.500 & 0.000 \\
\hline 64.2 & 2 & 2 & $\begin{array}{l}\text { Foraminiferal nanno- } \\
\text { fossil chalk ooze }\end{array}$ & 101.25 & 1.60 & 66.0 & 1.452 & 2319 & 68.887 & 0.153 & 3.705 & 0.000 & 3.705 & 0.500 & 0.000 \\
\hline 64.0 & 2 & 4 & $\begin{array}{l}\text { Foraminiferal nanno- } \\
\text { fossil chalk ooze }\end{array}$ & 104.25 & 1.66 & 62.2 & 1.462 & 2430 & & & & & & & \\
\hline 64.0 & 2 & 5 & $\begin{array}{l}\text { Foraminiferal nanno- } \\
\text { fossil chalk ooze }\end{array}$ & 105.75 & 1.68 & 61.1 & 1.483 & 2491 & & & & & & & \\
\hline 64.0 & 2 & 6 & $\begin{array}{l}\text { Foraminiferal nanno- } \\
\text { fossil chalk ooze }\end{array}$ & 107.25 & 1.67 & 61.9 & 1.459 & 2432 & 69.768 & 0.226 & 3.994 & 0.000 & 3.994 & 0.500 & 0.000 \\
\hline 64.0 & 3 & 1 & Nannofossil chalk ooze & 202.75 & 1.69 & 60.6 & 1.488 & 2511 & 70.368 & 0.254 & 4.095 & 0.000 & 4.095 & 0.500 & 0.000 \\
\hline 64.0 & 3 & 2 & Nannofossil chalk ooze & 204.25 & 1.72 & 59.0 & 1.529 & 2622 & 71.354 & 0.294 & 4.230 & 0.000 & 4.230 & 0.500 & 0.000 \\
\hline
\end{tabular}
*NOTES: Depth $=$ depth below sea floor, meters; $\rho_{\mathrm{B}}=$ saturated bulk density, gm $/ \mathrm{cm}^{3} ; \phi=$ porosity, fraction of total volume; $\mathrm{V}_{\mathrm{P}}=$ velocity, compressional wave, $\mathrm{km} / \mathrm{sec} ; \mathrm{Imped}$ -
ance $=\mathrm{gm} / \mathrm{cm}^{2} \sec \times 10^{2} ; \mathrm{K}_{\mathrm{G}}=$ weighted mean bulk modulus, dynes $/ \mathrm{cm}^{2} \times 10^{10} ; \mathrm{K}_{\mathrm{S}}=$ skeletal bulk modulus, dynes $/ \mathrm{cm}^{2} \times 10^{10} ; \mathrm{K}=\mathrm{bulk} \mathrm{modulus} \mathrm{sediment,} \mathrm{dynes} / \mathrm{cm}^{2} \times$ $10^{10} ; \mu=$ rigidity, dynes $/ \mathrm{cm}^{2} \times 10^{10} ; \lambda=$ Lame's constant, dynes $/ \mathrm{cm}^{2} \times 10^{10} ; \theta=$ Poisson's ratio; $\mathrm{V}_{\mathrm{S}}=$ velocity of shear wave in $\mathrm{km} / \mathrm{sec}$. 
TABLES 1 through 6 - Continued

\begin{tabular}{|c|c|c|c|c|c|c|c|c|c|c|c|c|c|c|c|}
\hline \multicolumn{3}{|c|}{ Identification } & \multirow[b]{2}{*}{ Lithology } & \multirow[b]{2}{*}{ Depth* } & \multirow[b]{2}{*}{$\rho_{\mathrm{B}}$} & \multirow[b]{2}{*}{$\phi$} & \multirow[b]{2}{*}{$\mathrm{V}_{\mathrm{P}}$} & \multirow{2}{*}{$\begin{array}{l}\text { Acoustic } \\
\text { Impedance }\end{array}$} & \multirow[b]{2}{*}{$\mathrm{K}_{\mathrm{G}}$} & \multirow[b]{2}{*}{$\mathrm{K}_{\mathrm{S}}$} & \multirow[b]{2}{*}{ K } & \multirow[b]{2}{*}{$\mu$} & \multirow[b]{2}{*}{$\lambda$} & \multirow[b]{2}{*}{$\theta$} & \multirow[b]{2}{*}{$\mathrm{v}_{\mathrm{S}}$} \\
\hline Hole & Core & Section & & & & & & & & & & & & & \\
\hline 64.0 & 3 & 3 & Nannofossil chalk ooze & 205.75 & 1.72 & 58.8 & 1.476 & 2537 & 68.042 & 0.302 & 4.247 & 0.000 & 4.247 & 0.500 & 0.000 \\
\hline 64.0 & 3 & 4 & Nannofossil chalk ooze & 207.25 & 1.69 & 60.8 & 1.480 & 2494 & 70.508 & 0.249 & 4.081 & 0.000 & 4.081 & 0.500 & 0.000 \\
\hline 64.0 & 3 & 5 & Nannofossil chalk ooze & 208.75 & 1.71 & 59.5 & 1.486 & 2537 & 69.980 & 0.282 & 4.187 & 0.000 & 4.187 & 0.500 & 0.000 \\
\hline 64.0 & 3 & 6 & Nannofossil chalk ooze & 210.25 & 1.70 & 59.7 & 1.506 & 2564 & 68.570 & 0.276 & 4.165 & 0.000 & 4.165 & 0.500 & 0.000 \\
\hline 64.0 & 4 & 2 & Nannofossil chalk ooze & 306.25 & 1.67 & 61.5 & 1.504 & 2518 & 68.817 & 0.234 & 4.025 & 0.000 & 4.025 & 0.500 & 0.000 \\
\hline 64.0 & 4 & 3 & Nannofossil chalk ooze & 307.75 & 1.72 & 59.0 & 1.507 & 2585 & 67.513 & 0.294 & 4.224 & 0.000 & 4.224 & 0.500 & 0.000 \\
\hline 64.0 & 4 & 4 & Nannofossil chalk ooze & 309.25 & 1.71 & 59.4 & 1.518 & 2592 & 62.720 & 0.284 & 4.182 & 0.000 & 4.182 & 0.500 & 0.000 \\
\hline 64.0 & 4 & 5 & Nannofossil chalk ooze & 310.75 & 1.71 & 59.2 & 1.530 & 2619 & 68.359 & 0.290 & 4.212 & 0.000 & 4.212 & 0.500 & 0.000 \\
\hline 64.0 & 4 & 6 & Nannofossil chalk ooze & 312.25 & 1.72 & 58.5 & 1.566 & 2699 & 69.768 & 0.310 & 4.277 & 0.073 & 4.228 & 0.492 & 0.206 \\
\hline 64.0 & 5 & 1 & Nannofossil chalk ooze & 409.75 & 1.75 & 57.2 & 1.539 & 2687 & 69.592 & 0.351 & 4.399 & 0.000 & 4.399 & 0.518 & 0.000 \\
\hline 64.0 & 5 & 2 & Nannofossil chalk ooze & 411.25 & 1.71 & 59.1 & & & & 0.294 & 4.223 & 0.618 & 3.811 & 0.430 & 0.601 \\
\hline 64.0 & 5 & 3 & Nannofossil chalk ooze & 412.75 & 1.71 & 59.2 & 1.544 & 2643 & & & & & & & \\
\hline 64.0 & 5 & 4 & Nannofossil chalk ooze & 414.25 & 1.75 & 57.1 & 1.582 & 2766 & 68.817 & 0.354 & 4.407 & 0047 & 4.376 & 0.495 & 0.164 \\
\hline 64.0 & 5 & 5 & Nannofossil chalk ooze & 415.75 & 1.78 & 55.4 & 1.616 & 2869 & 67.161 & 0.413 & 4.569 & 0.129 & 4.483 & 0.486 & 0.270 \\
\hline 64.0 & 5 & 6 & Nannofossil chalk ooze & 417.25 & 1.76 & 56.3 & 1.646 & 2896 & & & & & & & \\
\hline 64.0 & 6 & 1 & Nannofossil marl ooze & 505.75 & 1.79 & 54.4 & 1.626 & 2918 & 53.769 & 0.456 & 4.638 & 0.247 & 4.473 & 0.474 & 0.371 \\
\hline 64.0 & 6 & 2 & Nannofossil chalk ooze & 507.25 & 1.80 & 54.0 & 1.633 & 2939 & 66.209 & 0.472 & 4.721 & 0.238 & 4.563 & 0.475 & 0.363 \\
\hline 64.0 & 6 & 3 & Nannofossil chalk ooze & 508.75 & 1.79 & 54.4 & 1.661 & 2978 & 69.698 & 0.455 & 4.686 & 0.362 & 4.445 & 0.462 & 0.450 \\
\hline 64.0 & 6 & 4 & Nannofossil chalk ooze & 510.25 & 1.83 & 52.5 & 1.680 & 3067 & 68.923 & 0.542 & 4.902 & 0.275 & 4.719 & 0.473 & 0.388 \\
\hline 64.0 & 6 & 5 & Nannofossil chalk ooze & 511.75 & 1.84 & 51.7 & 1.675 & 3079 & 64.236 & 0.583 & 4.983 & 0.205 & 4.846 & 0.480 & 0.334 \\
\hline 64.0 & 6 & 6 & Nannofossil chalk ooze & 513.25 & 1.85 & 50.8 & 1.758 & 3258 & & & & & & & \\
\hline 64.0 & 7 & 2 & Nannofossil chalk & 612.25 & 1.78 & 55.4 & 1.616 & 2868 & 68.006 & 0.412 & 4.568 & 0.417 & 4.290 & 0.456 & 0.485 \\
\hline 64.0 & 7 & 3 & Nannofossil chalk & 613.75 & 1.76 & 56.2 & 1.592 & 2805 & 68.324 & 0.383 & 4.489 & 0.042 & 4.461 & 0.495 & 0.154 \\
\hline 64.0 & 7 & 4 & Nannofossil chalk & 615.25 & 1.79 & 54.8 & 1.663 & 2968 & 68.923 & 0.436 & 4.633 & 0.331 & 4.413 & 0.465 & 0.430 \\
\hline 64.0 & 7 & 5 & Nannofossil chalk & 616.75 & 1.74 & 57.5 & 1.606 & 2794 & 69.205 & 0.339 & 4.364 & 0.169 & 4.251 & 0.481 & 0.312 \\
\hline
\end{tabular}




\begin{tabular}{|c|c|c|c|c|c|c|c|c|c|c|c|c|c|c|c|}
\hline 64.0 & 7 & 6 & Nannofossil chalk & 618.25 & 1.72 & 58.6 & 1.657 & 2853 & 69.768 & 0.307 & 4.266 & 0.372 & 4.018 & 0.458 & 0.465 \\
\hline 64.0 & 8 & 2 & Nannofossil chalk & 707.25 & 1.92 & 47.1 & 1.902 & 3645 & 67.971 & 0.898 & 5.671 & 1.455 & 4.701 & 0.382 & 0.871 \\
\hline 64.0 & 8 & 3 & Nannofossil chalk & 708.75 & 1.92 & 46.9 & 1.949 & 3741 & 68.042 & 0.915 & 5.704 & 1.997 & 4.373 & 0.343 & 1.020 \\
\hline 64.0 & 10 & 1 & $\begin{array}{l}\text { Nannofossil chalk and } \\
\text { limestone }\end{array}$ & 848.75 & 1.87 & 50.1 & 1.941 & 3621 & 68.676 & 0.681 & 5.221 & 1.510 & 4.215 & 0.368 & 0.900 \\
\hline 64.0 & 10 & 2 & $\begin{array}{l}\text { Nannofossil chalk and } \\
\text { limestone }\end{array}$ & 850.25 & 1.95 & 45.2 & 2.073 & 4037 & 67.478 & 1.068 & 5.995 & 2.831 & 4.108 & 0.296 & 1.206 \\
\hline 64.1 & 1 & 1 & Nannofossil chalk ooze & 433.75 & 1.79 & 54.6 & 1.609 & 2879 & 69.663 & 0.445 & 4.659 & 0.006 & 4.655 & 0.499 & 0.057 \\
\hline 64.1 & 1 & 2 & Nannofossil chalk ooze & 435.25 & 1.78 & 55.0 & 1.606 & 2862 & 65.786 & 0.428 & 4.606 & 0.032 & 4.585 & 0.497 & 0.133 \\
\hline 64.1 & 1 & 3 & Nannofossil chalk ooze & 436.75 & 1.77 & 55.9 & 1.626 & 2874 & 62.121 & 0.393 & 4.501 & 0.233 & 4.346 & 0.475 & 0.363 \\
\hline 64.1 & 1 & 4 & Nannofossil chalk ooze & 438.25 & 1.78 & 55.1 & 1.656 & 2949 & 69.451 & 0.424 & 4.604 & 0.254 & 4.434 & 0.473 & 0.378 \\
\hline 64.1 & 1 & 5 & Nannofossil chalk ooze & 439.75 & 1.81 & 53.2 & 1.602 & 2904 & 69.698 & 0.508 & 4.821 & 0.000 & 4.821 & 0.500 & 0.000 \\
\hline 64.1 & 1 & 6 & Nannofossil chalk ooze & 441.25 & 1.76 & 56.6 & 1.609 & 2825 & 68.147 & 0.371 & 4.454 & 0.168 & 4.342 & 0.481 & 0.309 \\
\hline 64.1 & 2 & 2 & $\begin{array}{l}\text { Nannofossil chalk and } \\
\text { chalk ooze }\end{array}$ & 444.25 & 1.74 & 57.4 & 1.594 & 2776 & 67.654 & 0.342 & 4.369 & 0.068 & 4.325 & 0.492 & 0.197 \\
\hline 64.1 & 2 & 3 & $\begin{array}{l}\text { Nannofossil chalk and } \\
\text { chalk ooze }\end{array}$ & 445.75 & 1.79 & 54.7 & 1.583 & 2829 & 66.597 & 0.440 & 4.638 & 0.000 & 4.638 & 0.500 & 0.000 \\
\hline 64.1 & 2 & 4 & $\begin{array}{l}\text { Nannofossil chalk and } \\
\text { chalk ooze }\end{array}$ & 447.25 & 1.82 & 53.1 & 1.657 & 3007 & 68.923 & 0.514 & 4.833 & 0.213 & 4.691 & 0.478 & 0.342 \\
\hline 64.1 & 2 & 5 & $\begin{array}{l}\text { Nannofossil chalk and } \\
\text { chalk ooze }\end{array}$ & 448.75 & 1.79 & 54.4 & 1.626 & 2915 & 68.923 & 0.456 & 4.685 & 0.063 & 4.643 & 0.493 & 0.188 \\
\hline 64.1 & 2 & 6 & $\begin{array}{l}\text { Nannofossil chalk and } \\
\text { chalk ooze }\end{array}$ & 450.25 & 1.81 & 53.2 & 1.613 & 2923 & 69.945 & 0.506 & 4.815 & 0.000 & 4.815 & 0.500 & 0.000 \\
\hline 64.1 & 3 & 1 & $\begin{array}{l}\text { Nannofossil chalk and } \\
\text { chalk ooze }\end{array}$ & 451.75 & 1.82 & 53.1 & 1.580 & 2868 & 69.169 & 0.512 & 4.829 & 0.000 & 4.829 & 0.500 & 0.000 \\
\hline 64.1 & 3 & 2 & $\begin{array}{l}\text { Nannofossil chalk and } \\
\text { chalk ooze }\end{array}$ & 453.25 & 1.77 & 55.5 & 1.571 & 2787 & 68.500 & 0.409 & 4.559 & 0.000 & 4.559 & 0.500 & 0.000 \\
\hline 64.1 & 3 & 4 & $\begin{array}{l}\text { Nannofossil chalk and } \\
\text { chalk ooze }\end{array}$ & 456.25 & 1.81 & 53.6 & 1.621 & 2929 & 69.275 & 0.491 & 4.777 & 0.085 & 4.721 & 0.491 & 0.216 \\
\hline
\end{tabular}

*NOTES: Depth $=$ depth below sea floor, meters; $\rho_{\mathrm{B}}=$ saturated bulk density, $\mathrm{gm} / \mathrm{cm}^{3} ; \phi=$ porosity, fraction of total volume; $\mathrm{V}_{\mathrm{P}}=$ velocity, compressional wave, $\mathrm{km} / \mathrm{sec} ; \mathrm{Imped}$ ance $=\mathrm{gm} / \mathrm{cm}^{2} \sec \times 10^{2} ; \mathrm{K}_{\mathrm{G}}=$ weighted mean bulk modulus, dynes $/ \mathrm{cm}^{2} \times 10^{10} ; \mathrm{K}_{\mathrm{S}}=$ skeletal bulk modulus, dynes $/ \mathrm{cm}^{2} \times 10^{10} ; \mathrm{K}=$ bulk modulus sediment, dynes $/ \mathrm{cm}^{2} \times$ $10^{10} ; \mu=$ rigidity, dynes $/ \mathrm{cm}^{2} \times 10^{10} ; \lambda=$ Lame's constant, dynes $/ \mathrm{cm}^{2} \times 10^{10} ; \theta=$ Poisson's ratio; $\mathrm{V}_{\mathrm{S}}=$ velocity of shear wave in $\mathrm{km} / \mathrm{sec}$. 
TABLES 1 through 6 - Continued

\begin{tabular}{|c|c|c|c|c|c|c|c|c|c|c|c|c|c|c|c|}
\hline \multicolumn{3}{|c|}{ Lithology } & \multirow[b]{2}{*}{ Lithology } & \multirow[b]{2}{*}{ Depth* } & \multirow[b]{2}{*}{$\rho_{\mathrm{B}}$} & \multirow[b]{2}{*}{$\phi$} & \multirow[b]{2}{*}{$\mathrm{V}_{\mathrm{P}}$} & \multirow{2}{*}{$\begin{array}{l}\text { Acoustic } \\
\text { Impedance }\end{array}$} & \multirow[b]{2}{*}{$\mathrm{K}_{\mathrm{G}}$} & \multirow[b]{2}{*}{$\mathrm{K}_{\mathrm{S}}$} & \multirow[b]{2}{*}{$\mathrm{K}$} & \multirow[b]{2}{*}{$\mu$} & \multirow[b]{2}{*}{$\lambda$} & \multirow[b]{2}{*}{$\theta$} & \multirow[b]{2}{*}{$\mathrm{v}_{\mathrm{S}}$} \\
\hline Hole & Core & Section & & & & & & & & & & & & & \\
\hline 64.1 & 3 & 5 & $\begin{array}{l}\text { Nannofossil chalk and } \\
\text { chalk ooze }\end{array}$ & 457.75 & 1.77 & 55.6 & 1.641 & 2909 & 70.438 & 0.407 & 4.560 & 0.178 & 4.442 & 0.481 & 0.317 \\
\hline 64.1 & 4 & 1 & $\begin{array}{l}\text { Nannofossil chalk and } \\
\text { chalk ooze }\end{array}$ & 461.75 & 1.75 & 56.8 & 1.595 & 2794 & 68.218 & 0.362 & 4.428 & 0.270 & 4.248 & 0.470 & 0.392 \\
\hline 64.1 & 4 & 2 & $\begin{array}{l}\text { Nannofossil chalk and } \\
\text { chalk ooze }\end{array}$ & 463.25 & 1.76 & 56.2 & 1.580 & 2786 & 69.522 & 0.384 & 4.493 & 0.019 & 4.481 & 0.498 & 0.104 \\
\hline 64.1 & 4 & 3 & $\begin{array}{l}\text { Nannofossil chalk and } \\
\text { chalk ooze }\end{array}$ & 464.75 & 1.77 & 55.8 & 1.617 & 2861 & & & & & & & \\
\hline 64.1 & 4 & 4 & $\begin{array}{l}\text { Nannofossil chalk and } \\
\text { chalk ooze }\end{array}$ & 466.25 & 1.76 & 56.6 & 1.555 & 2731 & 68.183 & 0.370 & 4.451 & 0.000 & 4.451 & 0.500 & 0.000 \\
\hline 64.1 & 4 & 5 & $\begin{array}{l}\text { Nannofossil chalk and } \\
\text { chalk ooze }\end{array}$ & 467.75 & 1.80 & 54.0 & 1.697 & 3052 & 67.302 & 0.469 & 4.717 & 0.454 & 4.414 & 0.453 & 0.502 \\
\hline 64.1 & 4 & 6 & $\begin{array}{l}\text { Nannofossil chalk and } \\
\text { chalk ooze }\end{array}$ & 469.25 & 1.82 & 52.8 & 1.727 & 3144 & 68.535 & 0.527 & 4.864 & 0.575 & 4.481 & 0.443 & 0.562 \\
\hline 64.1 & 5 & 2 & $\begin{array}{l}\text { Nannofossil chalk and } \\
\text { chalk ooze }\end{array}$ & 472.25 & 1.77 & 55.6 & 1.526 & 2704 & 67.654 & 0.404 & 4.545 & 0.000 & 4.545 & 0.500 & 0.000 \\
\hline 64.1 & 5 & 3 & $\begin{array}{l}\text { Nannofossil chalk and } \\
\text { chalk ooze }\end{array}$ & 473.75 & 1.80 & 54.0 & 1.569 & 2822 & 67.936 & 0.469 & 4.718 & 0.000 & 4.718 & 0.500 & 0.000 \\
\hline 64.1 & 5 & 4 & $\begin{array}{l}\text { Nannofossil chalk and } \\
\text { chalk ooze }\end{array}$ & 475.25 & 1.83 & 52.3 & 1.549 & 2831 & 69.487 & 0.552 & 4.927 & 0.000 & 4.927 & 0.500 & 0.000 \\
\hline 64.1 & 5 & 5 & $\begin{array}{l}\text { Nannofossil chalk and } \\
\text { chalk ooze }\end{array}$ & 476.75 & 1.85 & 50.9 & 1.567 & 2901 & 70.227 & 0.630 & 5.112 & 0.000 & 5.112 & 0.500 & 0.000 \\
\hline 64.1 & 5 & 6 & $\begin{array}{l}\text { Nannofossil chalk and } \\
\text { chalk ooze }\end{array}$ & 478.25 & 1.83 & 52.4 & 1.585 & 2896 & 68.923 & 0.550 & 4.920 & 0.000 & 4.920 & 0.500 & 0.000 \\
\hline 64.1 & 6 & 1 & $\begin{array}{l}\text { Nannofossil chalk and } \\
\text { limestone }\end{array}$ & 565.75 & 1.85 & 51.2 & 1.710 & 3158 & 68.923 & 0.611 & 5.065 & 0.333 & 4.843 & 0.468 & 0.425 \\
\hline 64.1 & 6 & 2 & $\begin{array}{l}\text { Nannofossil chalk and } \\
\text { limestone }\end{array}$ & 567.25 & 1.82 & 52.9 & 1.702 & 3094 & 68.535 & 0.523 & 4.853 & 0.399 & 4.588 & 0.460 & 0.468 \\
\hline
\end{tabular}




\begin{tabular}{|c|c|c|c|c|c|c|c|c|c|c|c|c|c|c|c|}
\hline 64.1 & 6 & 3 & $\begin{array}{l}\text { Nannofossil chalk and } \\
\text { limestone }\end{array}$ & 568.75 & 1.82 & 52.6 & 1.762 & 3211 & 68.394 & 0.535 & 4.882 & 0.692 & 4.421 & 0.432 & 0.616 \\
\hline 64.1 & 6 & 4 & $\begin{array}{l}\text { Nannofossil chalk and } \\
\text { limestone }\end{array}$ & 570.25 & 1.83 & 52.1 & 1.749 & 3204 & 69.980 & 0.563 & 4.956 & 0.612 & 4.548 & 0.441 & 0.578 \\
\hline 64.1 & 7 & 2 & $\begin{array}{l}\text { Nannofossil chalk and } \\
\text { limestone }\end{array}$ & 663.25 & 1.84 & 51.6 & 1.699 & 3124 & 66.244 & 0.587 & 5.000 & 0.340 & 4.773 & 0.467 & 0.430 \\
\hline 64.1 & 7 & 3 & $\begin{array}{l}\text { Nannofossil chalk and } \\
\text { limestone }\end{array}$ & 664.75 & 1.84 & 51.5 & 1.726 & 3178 & 67.407 & 0.593 & 5.017 & 0.408 & 4.745 & 0.460 & 0.471 \\
\hline 64.1 & 7 & 4 & $\begin{array}{l}\text { Nannofossil chalk and } \\
\text { limestone }\end{array}$ & 666.25 & 1.81 & 53.6 & 1.718 & 3104 & 69.698 & 0.490 & 4.775 & 0.722 & 4.294 & 0.428 & 0.632 \\
\hline 64.1 & 8 & 2 & Nannofossil chalk & 748.25 & 1.89 & 48.7 & 1.880 & 3551 & 69.169 & 0.774 & 5.422 & 1.183 & 4.634 & 0.398 & 0.791 \\
\hline 64.1 & 9 & 1 & Nannofossil chalk & 911.75 & 1.85 & 51 & 1.780 & 3290 & 68.394 & 0.617 & 5.076 & 0.850 & 4.509 & 0.421 & 0.678 \\
\hline 64.1 & 9 & 2 & Nannofossil chalk & 913.25 & 1.84 & 51.5 & 1.723 & 3173 & 70.121 & 0.595 & 5.029 & 0.459 & 4.724 & 0.456 & 0.499 \\
\hline 64.1 & 9 & 3 & Nannofossil chalk & 914.75 & 1.88 & 49.0 & 1.768 & 3330 & 70.156 & 0.752 & 5.379 & 0.483 & 5.058 & 0.456 & 0.506 \\
\hline 64.1 & 10 & 1 & Nannofossil chalk & 969.75 & 1.84 & 51.7 & 1.802 & 3314 & 66.808 & 0.586 & 4.998 & 0.916 & 4.388 & 0.414 & 0.706 \\
\hline 64.1 & 10 & 2 & Nannofossil chalk & 971.25 & 1.88 & 49.4 & 1.790 & 3358 & 67.302 & 0.721 & 5.302 & 0.572 & 4.920 & 0.448 & 0.552 \\
\hline \multicolumn{16}{|c|}{ Table 5. Site 65} \\
\hline 65.0 & 2 & 2 & Radiolarian ooze & 12.25 & 1.14 & 91.2 & 1.473 & 1678 & 34.600 & 0.005 & 2.614 & 0.000 & 2.614 & 0.500 & 0.000 \\
\hline 65.0 & 2 & 3 & Radiolarian ooze & 13.75 & 1.14 & 90.9 & 1.479 & 1692 & 34.600 & 0.005 & 2.624 & 0.000 & 2.624 & 0.500 & 0.000 \\
\hline 65.0 & 2 & 4 & Radiolarian ooze & 15.25 & 1.14 & 91.6 & 1.467 & 1665 & 34.600 & 0.004 & 2.605 & 0.000 & 2.605 & 0.500 & 0.000 \\
\hline 65.0 & 2 & 5 & Radiolarian ooze & 16.75 & 1.14 & 91.1 & 1.502 & 1714 & 34.600 & 0.005 & 2.617 & 0000 & 2.617 & 0.500 & 0.000 \\
\hline 65.0 & 3 & 1 & Radiolarian ooze & 19.75 & 1.15 & 90.7 & 1.492 & 1709 & 34.600 & 0.005 & 2.627 & 0.000 & 2.627 & 0.500 & 0.000 \\
\hline 65.0 & 3 & 2 & Radiolarian ooze & 21.25 & 1.14 & 91.2 & 1.478 & 1683 & 34.600 & 0.005 & 2.616 & 0.000 & 2.616 & 0.500 & 0.000 \\
\hline 65.0 & 3 & 3 & Radiolarian ooze & 22.75 & 1.14 & 91.2 & 1.475 & 1680 & 34.600 & 0.005 & 2.616 & 0.000 & 2.616 & 0.500 & 0.000 \\
\hline 65.0 & 3 & 4 & Radiolarian ooze & 24.25 & 1.16 & 90.0 & 1.469 & 1697 & 34.600 & 0.005 & 2.648 & 0.000 & 2.648 & 0.500 & 0.000 \\
\hline 65.0 & 3 & 5 & Radiolarian ooze & 25.75 & 1.15 & 90.1 & 1.467 & 1691 & 34.600 & 0.005 & 2.644 & 0.000 & 2.644 & 0.500 & 0.000 \\
\hline 65.0 & 5 & 2 & Radiolarian ooze & 39.25 & 1.15 & 89.8 & 1.489 & 1718 & 34.600 & 0.005 & 2.653 & 0.000 & 2.653 & 0.500 & 0.000 \\
\hline
\end{tabular}

*NOTES: Depth $=$ depth below sea floor, meters; $\rho_{\mathrm{B}}=$ saturated bulk density, $\mathrm{gm} / \mathrm{cm}^{3} ; \phi=$ porosity, fraction of total volume $; \mathrm{V}_{\mathrm{P}}=\mathrm{velocity}$, compressional wave, $\mathrm{km} / \mathrm{sec} ;$ Impedance $=\mathrm{gm} / \mathrm{cm}^{2} \sec \times 10^{2} ; \mathrm{K}_{\mathrm{G}}=$ weighted mean bulk modulus, dynes $/ \mathrm{cm}^{2} \times 10^{10} ; \mathrm{K}_{\mathrm{S}}=$ skeletal bulk modulus, dynes $/ \mathrm{cm}^{2} \times 10^{10} ; \mathrm{K}=$ bulk modulus sediment, dynes $/ \mathrm{cm}^{2} \times$ $10^{10} ; \mu=$ rigidity, dynes $/ \mathrm{cm}^{2} \times 10^{10} ; \lambda=$ Lame's constant, dynes $/ \mathrm{cm}^{2} \times 10^{10} ; \theta=$ Poisson's ratio; $\mathrm{V}_{\mathrm{S}}=$ velocity of shear wave in $\mathrm{km} / \mathrm{sec}$. 
TABLES 1 through 6 - Continued

\begin{tabular}{|c|c|c|c|c|c|c|c|c|c|c|c|c|c|c|c|}
\hline \multicolumn{3}{|c|}{ Identification } & \multirow[b]{2}{*}{ Lithology } & \multirow[b]{2}{*}{ Depth* } & \multirow[b]{2}{*}{$\rho_{\mathrm{B}}$} & \multirow[b]{2}{*}{$\phi$} & \multirow[b]{2}{*}{$V_{P}$} & \multirow{2}{*}{$\begin{array}{l}\text { Acoustic } \\
\text { Impedance }\end{array}$} & \multirow[b]{2}{*}{$\mathrm{K}_{\mathrm{G}}$} & \multirow[b]{2}{*}{$\mathrm{K}_{\mathrm{S}}$} & \multirow[b]{2}{*}{ K } & \multirow[b]{2}{*}{$\mu$} & \multirow[b]{2}{*}{$\lambda$} & \multirow[b]{2}{*}{$\theta$} & \multirow[b]{2}{*}{$\mathrm{v}_{\mathrm{S}}$} \\
\hline Hole & Core & Section & & & & & & & & & & & & & \\
\hline 65.0 & 5 & 3 & Radiolarian ooze & 40.75 & 1.15 & 89.9 & 1.467 & 1691 & & & & & & & \\
\hline 65.0 & 5 & 4 & Radiolarian ooze & 42.25 & 1.16 & 89.4 & 1.454 & 1685 & 34.600 & 0.005 & 2.663 & 0.000 & 2.663 & 0.500 & 0.000 \\
\hline 65.0 & 5 & 5 & Radiolarian ooze & 43.75 & 1.14 & 90.8 & 1.464 & 1672 & 34.600 & 0.005 & 2.627 & 0.000 & 2.627 & 0.500 & 0.000 \\
\hline 65.0 & 6 & 2 & Radiolarian ooze & 48.25 & 1.15 & 89.9 & 1.480 & 1703 & 34.600 & 0.005 & 2.651 & 0.000 & 2.651 & 0.500 & 0.000 \\
\hline 65.0 & 7 & 2 & Radiolarian ooze & 57.25 & 1.16 & 89.1 & 1.474 & 1710 & 34.600 & 0.005 & 2.673 & 0.000 & 2.673 & 0.500 & 0.000 \\
\hline 65.0 & 7 & 3 & Radiolarian ooze & 58.75 & 1.17 & 88.4 & 1.486 & 1737 & 34.600 & 0.006 & 2.694 & 0.000 & 2.694 & 0.500 & 0.000 \\
\hline 65.0 & 7 & 4 & Radiolarian ooze & 60.25 & 1.15 & 90.3 & 1.478 & 1693 & 34.600 & 0.005 & 2.640 & 0.000 & 2.640 & 0.500 & 0.000 \\
\hline 65.0 & 7 & 5 & Radiolarian ooze & 61.75 & 1.14 & 91.1 & 1.468 & 1667 & 34.600 & 0.005 & 2.618 & 0.000 & 2.618 & 0.500 & 0.000 \\
\hline 65.0 & 8 & 1 & Radiolarian ooze & 64.75 & 1.18 & 87.2 & 1.460 & 1728 & 34.600 & 0.007 & 2.728 & 0.000 & 2.728 & 0.500 & 0.000 \\
\hline 65.0 & 8 & 2 & Radiolarian ooze & 66.25 & 1.19 & 86.9 & 1.474 & 1749 & 34.600 & 0.007 & 2.735 & 0.000 & 2.735 & 0.500 & 0.000 \\
\hline 65.0 & 8 & 3 & Radiolarian ooze & 67.75 & 1.21 & 84.7 & 1.473 & 1786 & 34.600 & 0.009 & 2.803 & 0.000 & 2.803 & 0.500 & 0.000 \\
\hline 65.0 & 8 & 4 & Radiolarian ooze & 69.25 & 1.17 & 88.4 & 1.468 & 1714 & 34.600 & 0.006 & 2.691 & 0.000 & 2.691 & 0.500 & 0.000 \\
\hline 65.0 & 8 & 5 & Radiolarian ooze & 70.75 & 1.20 & 85.5 & 1.473 & 1772 & 34.600 & 0.008 & 2.776 & 0.000 & 2.776 & 0.500 & 0.000 \\
\hline 65.0 & 8 & 6 & Radiolarian ooze & 72.25 & 1.19 & 86.4 & 1.482 & 1766 & 34.600 & 0.007 & 2.752 & 0.000 & 2.752 & 0.500 & 0.000 \\
\hline 65.0 & 9 & 1 & Radiolarian ooze & 74.75 & 1.22 & 84.3 & 1.448 & 1762 & 34.600 & 0.009 & 2.813 & 0.000 & 2.813 & 0.500 & 0.000 \\
\hline 65.0 & 9 & 2 & Radiolarian ooze & 76.25 & 1.21 & 85.0 & 1.459 & 1761 & 34.600 & 0.008 & 2.792 & 0.000 & 2.792 & 0.500 & 0.000 \\
\hline 65.0 & 9 & 3 & Radiolarian ooze & 77.75 & 1.21 & 84.5 & 1.483 & 1800 & 34.600 & 0.009 & 2.808 & 0.000 & 2.808 & 0.500 & 0.000 \\
\hline 65.0 & 9 & 4 & Radiolarian ooze & 79.25 & 1.19 & 86.7 & 1.484 & 1762 & & & & & & & \\
\hline 65.0 & 9 & 5 & Radiolarian ooze & 80.75 & 1.21 & 85.3 & 1.456 & 1754 & 34.600 & 0.008 & 2.785 & 0.000 & 2.785 & 0.500 & 0.000 \\
\hline 65.0 & 9 & 6 & Radiolarian ooze & 82.25 & 1.22 & 84.2 & 1.442 & 1755 & & & & & & & \\
\hline 65.0 & 10 & 2 & Radiolarian ooze & 85.25 & 1.21 & 84.7 & 1.451 & 1756 & & & & & & & \\
\hline 65.0 & 10 & 3 & Radiolarian ooze & 86.75 & 1.21 & 84.5 & 1.454 & 1763 & 34.600 & 0.009 & 2.809 & 0.000 & 2.809 & 0.500 & 0.000 \\
\hline 65.0 & 10 & 4 & Radiolarian ooze & 88.25 & 1.21 & 84.7 & 1.472 & 1781 & 34.600 & 0.009 & 2.803 & 0.000 & 2.803 & 0.500 & 0.000 \\
\hline 65.0 & 11 & 2 & Radiolarian ooze & 94.25 & 1.16 & 89.0 & 1.463 & 1693 & 34.600 & 0.006 & 2.677 & 0.000 & 2.677 & 0.500 & 0.000 \\
\hline 65.0 & 11 & 3 & Radiolarian ooze & 95.75 & 1.16 & 88.7 & 1.487 & 1726 & 34.600 & 0.006 & 2.685 & 0.000 & 2.685 & 0.500 & 0.000 \\
\hline
\end{tabular}




\begin{tabular}{|c|c|c|c|c|c|c|c|c|c|c|c|c|c|c|c|}
\hline 65.0 & 11 & 4 & Radiolarian ooze & 97.25 & 1.15 & 89.7 & 1.477 & 1697 & 34.600 & 0.005 & 2.657 & 0.000 & 2.657 & 0.500 & 0.000 \\
\hline 65.0 & 11 & 5 & Radiolarian ooze & 98.75 & 1.13 & 91.3 & 1.470 & 1658 & 34.600 & 0.005 & 2.611 & 0.000 & 2.611 & 0.500 & 0.000 \\
\hline 65.0 & 11 & 6 & Radiolarian ooze & 100.25 & 1.12 & 92.3 & 1.454 & 1623 & 34.600 & 0.004 & 2.584 & 0.000 & 2.584 & 0.500 & 0.000 \\
\hline 65.0 & 12 & 1 & Radiolarian ooze & 101.75 & 1.10 & 93.7 & 1.483 & 1631 & 34.600 & 0.004 & 2.549 & 0.000 & 2.549 & 0.500 & 0.000 \\
\hline 65.0 & 12 & 2 & Radiolarian ooze & 103.25 & 1.15 & 89.2 & 1.493 & 1720 & 34.600 & 0.006 & 2.669 & 0.000 & 2.669 & 0.500 & 0.000 \\
\hline 65.0 & 12 & 3 & Radiolarian ooze & 104.75 & 1.16 & 89.0 & 1.500 & 1732 & 34.600 & 0.006 & 2.676 & 0.000 & 2.676 & 0.500 & 0.000 \\
\hline 65.0 & 12 & 4 & Radiolarian ooze & 106.25 & 1.23 & 82.8 & 1.437 & 1765 & 34.600 & 0.010 & 2.863 & 0.000 & 2.863 & 0.500 & 0.000 \\
\hline 65.0 & 12 & 5 & Radiolarian ooze & 107.75 & 1.17 & 87.8 & 1.492 & 1743 & 34.600 & 0.006 & 2.709 & 0.000 & 2.709 & 0.500 & 0.000 \\
\hline 65.0 & 12 & 6 & Radiolarian ooze & 109.25 & 1.15 & 89.1 & 1.491 & 1720 & 34.600 & 0.006 & 2.673 & 0.000 & 2.673 & 0.500 & 0.000 \\
\hline 65.0 & 13 & 1 & Radiolarian ooze & 110.75 & 1.12 & 92.0 & 1.499 & 1678 & 34.600 & 0.004 & 2.594 & 0.000 & 2.594 & 0.500 & 0.000 \\
\hline 65.0 & 13 & 2 & Radiolarian ooze & 112.25 & 1.13 & 91.2 & 1.491 & 1680 & 34.600 & 0.005 & 2.615 & 0.000 & 2.615 & 0.500 & 0.000 \\
\hline 65.0 & 13 & 3 & Radiolarian ooze & 113.75 & 1.15 & 88.8 & 1.527 & 1762 & 34.600 & 0.006 & 2.680 & 0.016 & 2.670 & 0.497 & 0.117 \\
\hline 65.0 & 13 & 4 & Radiolarian ooze & 115.25 & 1.15 & 88.9 & 1.493 & 1723 & 34.600 & 0.006 & 2.678 & 0.000 & 2.678 & 0.500 & 0.000 \\
\hline 65.0 & 13 & 5 & Radiolarian ooze & 116.75 & 1.16 & 88.4 & 1.492 & 1729 & 34.600 & 0.006 & 2.691 & 0.000 & 2.691 & 0.500 & 0.000 \\
\hline 65.0 & 13 & 6 & Radiolarian ooze & 118.25 & 1.17 & 87.7 & 1.533 & 1791 & 34.600 & 0.006 & 2.713 & 0.053 & 2.678 & 0.490 & 0.214 \\
\hline 65.0 & 14 & 1 & Radiolarian ooze & 119.75 & 1.16 & 88.5 & 1.486 & 1722 & 34.600 & 0.006 & 2.691 & 0.000 & 2.691 & 0.500 & 0.000 \\
\hline 65.0 & 14 & 2 & Radiolarian ooze & 121.25 & 1.18 & 86.0 & 1.505 & 1780 & 34.600 & 0.008 & 2.763 & 0.000 & 2.763 & 0.500 & 0.000 \\
\hline 65.0 & 14 & 3 & Radiolarian ooze & 122.75 & 1.24 & 80.6 & 1.514 & 1883 & 34.600 & 0.013 & 2.936 & 0.000 & 2.936 & 0.500 & 0.000 \\
\hline 65.0 & 14 & 4 & $\begin{array}{l}\text { Calcareous radio- } \\
\text { larian ooze }\end{array}$ & 124.25 & 1.29 & 76.9 & 1.490 & 1917 & 34.600 & 0.019 & 3.070 & 0.000 & 3.070 & 0.500 & 0.000 \\
\hline 65.0 & 14 & 5 & $\begin{array}{l}\text { Nannofossil radio- } \\
\text { larian ooze }\end{array}$ & 125.75 & 1.32 & 74.2 & 1.464 & 1928 & 34.600 & 0.024 & 3.173 & 0.000 & 3.173 & 0.500 & 0.000 \\
\hline 65.0 & 14 & 6 & $\begin{array}{l}\text { Nannofossil radio- } \\
\text { larian ooze }\end{array}$ & 127.25 & 1.39 & 68.1 & 1.460 & 2025 & 34.600 & 0.044 & 3.446 & 0.000 & 3.446 & 0.500 & 0.000 \\
\hline 65.0 & 16 & 3 & $\begin{array}{l}\text { Nannofossil radio- } \\
\text { larian ooze }\end{array}$ & 140.75 & 1.27 & 77.3 & 1.508 & 1919 & 34.600 & 0.018 & 3.055 & 0.000 & 3.055 & 0.500 & 0.000 \\
\hline 65.0 & 16 & 4 & $\begin{array}{l}\text { Nannofossil radio- } \\
\text { larian ooze }\end{array}$ & 142.25 & 1.21 & 82.7 & 1.488 & 1807 & 34.600 & 0.011 & 2.866 & 0.000 & 2.866 & 0.500 & 0.000 \\
\hline
\end{tabular}

*NOTES: Depth $=$ depth below sea floor, meters; $\rho_{\mathrm{B}}=$ saturated bulk density, $\mathrm{gm} / \mathrm{cm}^{3} ; \phi=$ porosity, fraction of total volume; $\mathrm{V}_{\mathrm{P}}=$ velocity, compressional wave, $\mathrm{km} / \mathrm{sec} ; \mathrm{Imped}$ ance $=\mathrm{gm} / \mathrm{cm}^{2} \sec \times 10^{2} ; \mathrm{K}_{\mathrm{G}}=$ weighted mean bulk modulus, dynes $/ \mathrm{cm}^{2} \times 10^{10} ; \mathrm{K}_{\mathrm{S}}=$ skeletal bulk modulus, dynes $/ \mathrm{cm}^{2} \times 10^{10} ; \mathrm{K}=$ bulk modulus sediment, dynes $/ \mathrm{cm}^{2} \times$ $10^{10} ; \mu=$ rigidity, dynes $/ \mathrm{cm}^{2} \times 10^{10} ; \lambda=$ Lame's constant, dynes $/ \mathrm{cm}^{2} \times 10^{10} ; \theta=$ Poisson's ratio; $\mathrm{V}_{\mathrm{S}}=$ velocity of shear wave in $\mathrm{km} / \mathrm{sec}$. 
TABLES 1 through 6 - Continued

\begin{tabular}{|c|c|c|c|c|c|c|c|c|c|c|c|c|c|c|c|}
\hline \multicolumn{3}{|c|}{ Identification } & \multirow[b]{2}{*}{ Lithology } & \multirow[b]{2}{*}{ Depth* } & \multirow[b]{2}{*}{$\rho_{\mathrm{B}}$} & \multirow[b]{2}{*}{$\phi$} & \multirow[b]{2}{*}{$\mathrm{V}_{\mathrm{P}}$} & \multirow{2}{*}{$\begin{array}{l}\text { Acoustic } \\
\text { Impedance }\end{array}$} & \multirow[b]{2}{*}{$\mathrm{K}_{\mathrm{G}}$} & \multirow[b]{2}{*}{$\mathrm{K}_{\mathrm{S}}$} & \multirow[b]{2}{*}{$\mathrm{K}$} & \multirow[b]{2}{*}{$\mu$} & \multirow[b]{2}{*}{$\lambda$} & \multirow[b]{2}{*}{$\theta$} & \multirow[b]{2}{*}{$\mathrm{v}_{\mathrm{S}}$} \\
\hline Hole & Core & Section & & & & & & & & & & & & & \\
\hline 65.0 & 16 & 5 & $\begin{array}{l}\text { Nannofossil radio- } \\
\text { larian ooze }\end{array}$ & 143.75 & 1.19 & 85.2 & 1.476 & 1751 & 34.600 & 0.008 & 2.786 & 0.000 & 2.786 & 0.500 & 0.000 \\
\hline 65.0 & 16 & 6 & $\begin{array}{l}\text { Nannofossil radio- } \\
\text { larian ooze }\end{array}$ & 145.25 & 1.20 & 84.2 & 1.492 & 1787 & 34.600 & 0.009 & 2.819 & 0.000 & 2.819 & 0.500 & 0.000 \\
\hline 65.1 & 4 & 1 & Radiolarian ooze & 154.75 & 1.17 & 86.5 & 1.495 & 1747 & 34.600 & 0.007 & 2.748 & 0.000 & 2.748 & 0.500 & 0.000 \\
\hline 65.1 & 4 & 2 & Radiolarian ooze & 156.25 & 1.19 & 83.9 & 1.494 & 1778 & 34.600 & 0.009 & 2.826 & 0.000 & 2.826 & 0.500 & 0.000 \\
\hline 65.1 & 4 & 3 & Radiolarian ooze & 157.75 & 1.20 & 83.4 & 1.514 & 1810 & 34.600 & 0.010 & 2.845 & 0.000 & 2.845 & 0.500 & 0.000 \\
\hline 65.1 & 4 & 4 & Radiolarian ooze & 159.25 & 1.20 & 83.3 & 1.528 & 1829 & 34.600 & 0.010 & 2.848 & 0.000 & 2.848 & 0.500 & 0.000 \\
\hline 65.1 & 4 & 5 & Radiolarian ooze & 160.75 & 1.18 & 85.4 & 1.509 & 1773 & 34.600 & 0.008 & 2.780 & 0.000 & 2.780 & 0.500 & 0.000 \\
\hline 65.1 & 4 & 6 & Radiolarian ooze & 162.25 & 1.17 & 86.2 & 1.516 & 1769 & 34.600 & 0.007 & 2.756 & 0.000 & 2.756 & 0.500 & 0.000 \\
\hline 65.1 & 5 & 1 & $\begin{array}{l}\text { Radiolarian ooze } \\
\text { with chert }\end{array}$ & 162.75 & 1.23 & 79.9 & 1.486 & 1831 & 34.600 & 0.014 & 2.961 & 0.000 & 2.961 & 0.500 & 0.000 \\
\hline 65.1 & 5 & 2 & $\begin{array}{l}\text { Radiolarian ooze } \\
\text { with chert }\end{array}$ & 164.25 & 1.18 & 84.6 & 1.507 & 1777 & 34.600 & 0.009 & 2.805 & 0.000 & 2.805 & 0.500 & 0.000 \\
\hline 65.1 & 5 & 3 & $\begin{array}{l}\text { Radiolarian ooze } \\
\text { with chert }\end{array}$ & 165.75 & 1.19 & 83.2 & 1.509 & 1801 & 34.600 & 0.010 & 2.850 & 0.000 & 2.850 & 0.500 & 0.000 \\
\hline 65.1 & 5 & 4 & $\begin{array}{l}\text { Radiolarian ooze } \\
\text { with chert }\end{array}$ & 167.25 & 1.20 & 82.1 & 1.502 & 1809 & 34.600 & 0.011 & 2.885 & 0.000 & 2.885 & 0.500 & 0.000 \\
\hline \multicolumn{16}{|c|}{ Table 6. Site 66} \\
\hline 66.0 & 2 & 1 & Radiolarian ooze & 79.75 & 1.10 & 92.0 & 1.439 & 1588 & 34.600 & 0.004 & 2.592 & 0.000 & 2.592 & 0.500 & 0.000 \\
\hline 66.0 & 2 & 2 & Radiolarian ooze & 81.25 & 1.12 & 92.7 & 1.443 & 1609 & 34.600 & 0.004 & 2.575 & 0.000 & 2.575 & 0.500 & 0.000 \\
\hline 66.0 & 2 & 3 & Radiolarian ooze & 82.75 & 1.10 & 93.8 & 1.487 & 1639 & 34.600 & 0.004 & 2.548 & 0.000 & 2.548 & 0.500 & 0.000 \\
\hline 66.0 & 3 & 1 & Radiolarian ooze & 117.75 & 1.17 & 88.1 & 1.495 & 1752 & 34.600 & 0.006 & 2.700 & 0.000 & 2.700 & 0.500 & 0.000 \\
\hline 66.0 & 3 & 2 & Radiolarian ooze & 119.25 & 1.19 & 85.8 & 1.492 & 1779 & 34.600 & 0.008 & 2.768 & 0.000 & 2.768 & 0.500 & 0.000 \\
\hline 66.0 & 3 & 3 & Radiolarian ooze & 120.75 & 1.18 & 87.0 & 1.501 & 1769 & 34.600 & 0.007 & 2.732 & 0.000 & 2.732 & 0.500 & 0.000 \\
\hline 66.0 & 3 & 4 & Radiolarian ooze & 122.25 & 1.18 & 87.2 & 1.486 & 1748 & & & & & & & \\
\hline
\end{tabular}




\begin{tabular}{|c|c|c|c|c|c|c|c|c|c|c|c|c|c|c|c|}
\hline 66.0 & 3 & 5 & Radiolarian ooze & 123.75 & 1.18 & 86.9 & 1.503 & 1772 & & & & & & & \\
\hline 66.0 & 3 & 6 & Radiolarian ooze & 125.25 & 1.17 & 87.9 & 1.502 & 1754 & 34.600 & 0.006 & 2.708 & 0.000 & 2.708 & 0.500 & 0.000 \\
\hline 66.0 & 6 & 2 & Pelagic clay & 167.25 & 1.52 & 70.8 & 1.416 & 2158 & 50.000 & 0.053 & 3.364 & 0.000 & 3.364 & 0.500 & 0.000 \\
\hline 66.0 & 6 & 3 & Pelagic clay & 168.75 & 1.54 & 70.0 & 1.400 & 2153 & 50.000 & 0.057 & 3.404 & 0.000 & 3.404 & 0.500 & 0.000 \\
\hline 66.0 & 6 & 4 & Pelagic clay & 170.25 & 1.58 & 67.9 & 1.427 & 2248 & 50.000 & 0.071 & 3.514 & 0.000 & 3.514 & 0.500 & 0.000 \\
\hline 66.0 & 7 & 1 & Pelagic clay & 174.75 & 1.56 & 69.1 & 1.425 & 2216 & 50.000 & 0.063 & 3.453 & 0.000 & 3.453 & 0.500 & 0.000 \\
\hline 66.0 & 7 & 2 & Pelagic clay & 176.25 & 1.62 & 65.8 & 1.450 & 2350 & 50.000 & 0.087 & 3.628 & 0.000 & 3.628 & 0.500 & 0.000 \\
\hline 66.0 & 7 & 3 & Pelagic clay & 177.75 & 1.62 & 65.9 & 1.465 & 2371 & 50.000 & 0.086 & 3.622 & 0.000 & 3.622 & 0.500 & 0.000 \\
\hline 66.0 & 7 & 4 & Pelagic clay & 179.25 & 1.64 & 64.9 & 1.451 & 2375 & 50.000 & 0.098 & 3.681 & 0.000 & 3.681 & 0.500 & 0.000 \\
\hline 66.0 & 8 & 1 & Pelagic clay & 180.75 & 1.53 & 70.9 & 1.429 & 2187 & 50.000 & 0.053 & 3.360 & 0.000 & 3.360 & 0.500 & 0.000 \\
\hline 66.0 & 8 & 2 & Pelagic clay & 182.25 & 1.51 & 72.6 & 1.419 & 2145 & 50.000 & 0.045 & 3.283 & 0.000 & 3.283 & 0.500 & 0.000 \\
\hline 66.0 & 8 & 3 & Pelagic clay & 183.75 & 1.43 & 77.4 & 1.447 & 2063 & 50.000 & 0.028 & 3.080 & 0.000 & 3.080 & 0.500 & 0.000 \\
\hline 66.0 & 8 & 4 & Pelagic clay & 185.25 & 1.38 & 79.9 & 1.440 & 1990 & 50.000 & 0.022 & 2.985 & 0.000 & 2.985 & 0.500 & 0.000 \\
\hline 66.0 & 8 & 5 & Pelagic clay & 186.75 & 1.39 & 79.6 & 1.433 & 1986 & 50.000 & 0.022 & 2.994 & 0.000 & 2.994 & 0.500 & 0.000 \\
\hline 66.0 & 9 & 1 & Volcanic mud & 187.75 & 1.45 & 76.2 & 1.357 & 1963 & 50.000 & 0.031 & 3.127 & 0.000 & 3.127 & 0.500 & 0.000 \\
\hline 66.1 & 2 & 1 & Radiolarian ooze & 20.75 & 1.18 & 91.4 & 1.460 & 1727 & & & & & & & \\
\hline 66.1 & 2 & 2 & Radiolarian ooze & 22.25 & 1.18 & 90.1 & 1.468 & 1727 & 34.600 & 0.005 & 2.644 & 0.000 & 2.644 & 0.500 & 0.000 \\
\hline 66.1 & 2 & 3 & Radiolarian ooze & 23.75 & 1.17 & 90.9 & 1.428 & 1664 & 34.600 & 0.005 & 2.623 & 0.000 & 2.623 & 0.500 & 0.000 \\
\hline 66.1 & 2 & 5 & Radiolarian ooze & 26.75 & 1.17 & 90.5 & 1.460 & 1711 & 34.600 & 0.005 & 2.634 & 0.000 & 2.634 & 0.500 & 0.000 \\
\hline 66.1 & 2 & 6 & Radiolarian ooze & 28.25 & 1.16 & 91.2 & 1.462 & 1697 & 34.600 & 0.005 & 2.615 & 0.000 & 2.615 & 0.500 & 0.000 \\
\hline 66.1 & 3 & 3 & Radiolarian ooze & 32.75 & 1.19 & 86.4 & 1.504 & 1785 & 34.600 & 0.007 & 2.751 & 0.000 & 2.751 & 0.500 & 0.000 \\
\hline 66.1 & 4 & 2 & Radiolarian ooze & 40.25 & 1.18 & 88.1 & 1.495 & 1758 & 34.600 & 0.006 & 2.701 & 0.000 & 2.701 & 0.500 & 0.000 \\
\hline 66.1 & 4 & 3 & Radiolarian ooze & 41.75 & 1.18 & 88.1 & 1.488 & 1750 & 34.600 & 0.006 & 2.701 & 0.000 & 2.701 & 0.500 & 0.000 \\
\hline 66.1 & 6 & 2 & Radiolarian ooze & 58.25 & 1.17 & 88.9 & 1.475 & 1731 & 34.600 & 0.006 & 2.679 & 0.000 & 2.679 & 0.500 & 0.000 \\
\hline 66.1 & 6 & 3 & Radiolarian ooze & 59.75 & 1.19 & 87.8 & 1.443 & 1714 & 34.600 & 0.006 & 2.710 & 0.000 & 2.710 & 0.500 & 0.000 \\
\hline 66.1 & 6 & 4 & Radiolarian ooze & 61.25 & 1.20 & 86.7 & 1.477 & 1776 & 34.600 & 0.007 & 2.741 & 0.000 & 2.741 & 0.500 & 0.000 \\
\hline
\end{tabular}

*NOTES: Depth $=$ depth below sea floor, meters; $\rho_{\mathrm{B}}=$ saturated bulk density, $\mathrm{gm} / \mathrm{cm}^{3} ; \phi=$ porosity, fraction of total volume; $\mathrm{V}_{\mathrm{P}}=\mathrm{velocity}$, compressional wave, $\mathrm{km} / \mathrm{sec} ;$ Impedance $=\mathrm{gm} / \mathrm{cm}^{2} \sec \times 10^{2} ; \mathrm{K}_{\mathrm{G}}=$ weighted mean bulk modulus, dynes $/ \mathrm{cm}^{2} \times 10^{10} ; \mathrm{K}_{\mathrm{S}}=$ skeletal bulk modulus, dynes $/ \mathrm{cm}^{2} \times 10^{10} ; \mathrm{K}=$ bulk modulus sediment, dynes $/ \mathrm{cm}{ }^{2} \times$ $10^{10} ; \mu=$ rigidity, dynes $/ \mathrm{cm}^{2} \times 10^{10} ; \lambda=$ Lame's constant, dynes $/ \mathrm{cm}^{2} \times 10^{10} ; \theta=$ Poisson's ratio; $\mathrm{V}_{\mathrm{S}}=$ velocity of shear wave in $\mathrm{km} / \mathrm{sec}$. 


\begin{tabular}{|c|c|c|c|c|c|c|c|c|c|c|c|c|c|c|c|}
\hline \multicolumn{3}{|c|}{ Identification } & \multirow[b]{2}{*}{ Lithology } & \multirow[b]{2}{*}{ Depth* } & \multirow[b]{2}{*}{$\rho_{\mathrm{B}}$} & \multirow[b]{2}{*}{$\phi$} & \multirow[b]{2}{*}{$V_{P}$} & \multirow{2}{*}{$\begin{array}{l}\text { Acoustic } \\
\text { Impedance }\end{array}$} & \multirow[b]{2}{*}{$\mathrm{K}_{\mathrm{G}}$} & \multirow[b]{2}{*}{$\mathrm{K}_{\mathrm{S}}$} & \multirow[b]{2}{*}{$\mathrm{K}$} & \multirow[b]{2}{*}{$\mu$} & \multirow[b]{2}{*}{$\lambda$} & \multirow[b]{2}{*}{$\theta$} & \multirow[b]{2}{*}{$\mathrm{v}_{\mathrm{S}}$} \\
\hline Hole & Core & Section & & & & & & & & & & & & & \\
\hline 66.1 & 6 & 5 & Radiolarian ooze & 62.75 & 1.19 & 87.3 & 1.466 & 1751 & 34.600 & 0.007 & 2.725 & 0.000 & 2.725 & 0.500 & 0.000 \\
\hline 66.1 & 6 & 6 & Radiolarian ooze & 64.25 & 1.20 & 86.9 & 1.486 & 1783 & 34.600 & 0.007 & 2.735 & 0.000 & 2.735 & 0.500 & 0.000 \\
\hline 66.1 & 7 & 1 & Radiolarian ooze & 67.75 & 1.15 & 90.3 & 1.472 & 1699 & 34.600 & 0.005 & 2.638 & 0.000 & 2.638 & 0.500 & 0.000 \\
\hline 66.1 & 7 & 2 & Radiolarian ooze & 69.25 & 1.17 & 88.6 & 1.475 & 1724 & 34.600 & 0.006 & 2.687 & 0.000 & 2.687 & 0.500 & 0.000 \\
\hline 66.1 & 7 & 3 & Radiolarian ooze & 70.75 & 1.20 & 85.9 & 1.473 & 1774 & 34.600 & 0.008 & 2.767 & 0.000 & 2.767 & 0.500 & 0.000 \\
\hline 66.1 & 7 & 4 & Radiolarian ooze & 72.25 & 1.19 & 86.9 & 1.470 & 1751 & 34.600 & 0.007 & 2.735 & 0.000 & 2.735 & 0.500 & 0.000 \\
\hline 66.1 & 7 & 5 & Radiolarian ooze & 73.75 & 1.22 & 85.0 & 1.472 & 1789 & 34.600 & 0.008 & 2.793 & 0.000 & 2.793 & 0.500 & 0.000 \\
\hline 66.1 & 7 & 6 & Radiolarian ooze & 75.25 & 1.17 & 88.7 & 1.467 & 1713 & 34.600 & 0.006 & 2.684 & 0.000 & 2.684 & 0.500 & 0.000 \\
\hline 66.1 & 8 & 1 & Radiolarian ooze & 76.75 & 1.19 & 86.8 & 1.489 & 1775 & 34.600 & 0.007 & 2.738 & 0.000 & 2.738 & 0.500 & 0.000 \\
\hline 66.1 & 8 & 2 & Radiolarian ooze & 78.25 & 1.20 & 85.9 & 1.489 & 1791 & 34.600 & 0.008 & 2.764 & 0.000 & 2.764 & 0.500 & 0.000 \\
\hline 66.1 & 8 & 3 & Radiolarian ooze & 79.75 & 1.18 & 87.4 & 1.483 & 1756 & 34.600 & 0.007 & 2.720 & 0.000 & 2.720 & 0.500 & 0.000 \\
\hline 66.1 & 8 & 6 & Radiolarian ooze & 84.25 & 1.20 & 86.5 & 1478 & 1767 & 34.600 & 0.007 & 2.747 & 0.000 & 2.747 & 0.500 & 0.000 \\
\hline
\end{tabular}

*NOTES: Depth $=$ depth below sea floor, meters; $\rho_{\mathrm{B}}=$ saturated bulk density, gm $/ \mathrm{cm}^{3} ; \phi=$ porosity, fraction of total volume; $\mathrm{V}_{\mathrm{P}}=$ velocity, compressional wave, $\mathrm{km} / \mathrm{sec} ;$ Impedance $=\mathrm{gm} / \mathrm{cm}^{2} \sec \times 10^{2} ; \mathrm{K}_{\mathrm{G}}=$ weighted mean bulk modulus, dynes $/ \mathrm{cm}^{2} \times 10^{10} ; \mathrm{K}_{\mathrm{S}}=$ skeletal bulk modulus, dynes $/ \mathrm{cm}^{2} \times 10^{10} ; \mathrm{K}=$ bulk modulus sediment, dynes $/ \mathrm{cm}{ }^{2} \times$ $10^{10} ; \mu=$ rigidity, dynes $/ \mathrm{cm}^{2} \times 10^{10} ; \lambda=$ Lame's constant, dynes $/ \mathrm{cm}^{2} \times 10^{10} ; \theta=$ Poisson's ratio; $V_{\mathrm{S}}=$ velocity of shear wave in $\mathrm{km} / \mathrm{sec}$. 


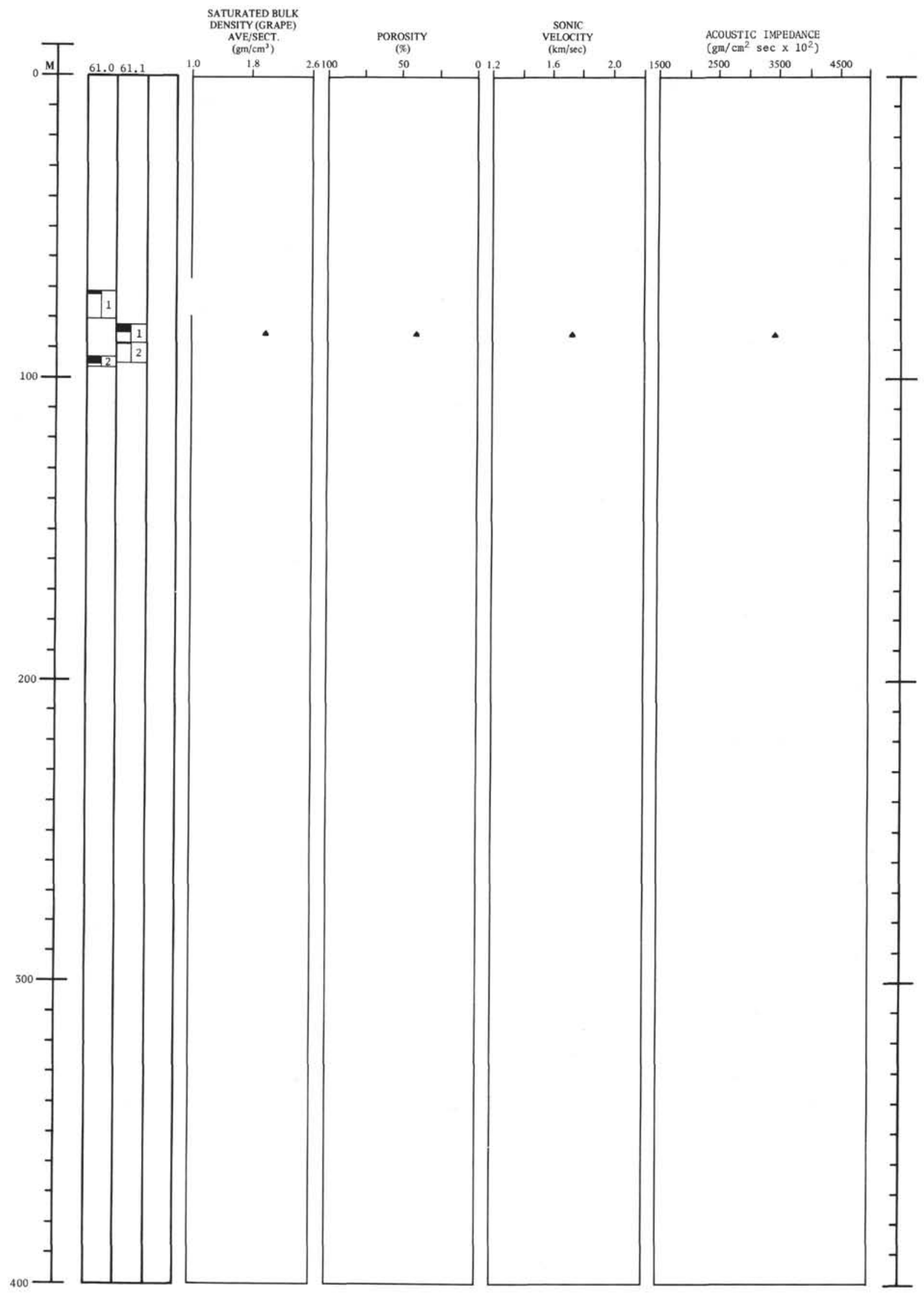

Figure 1. Sound velocity, elastic constants, and related properties, Site 61. 


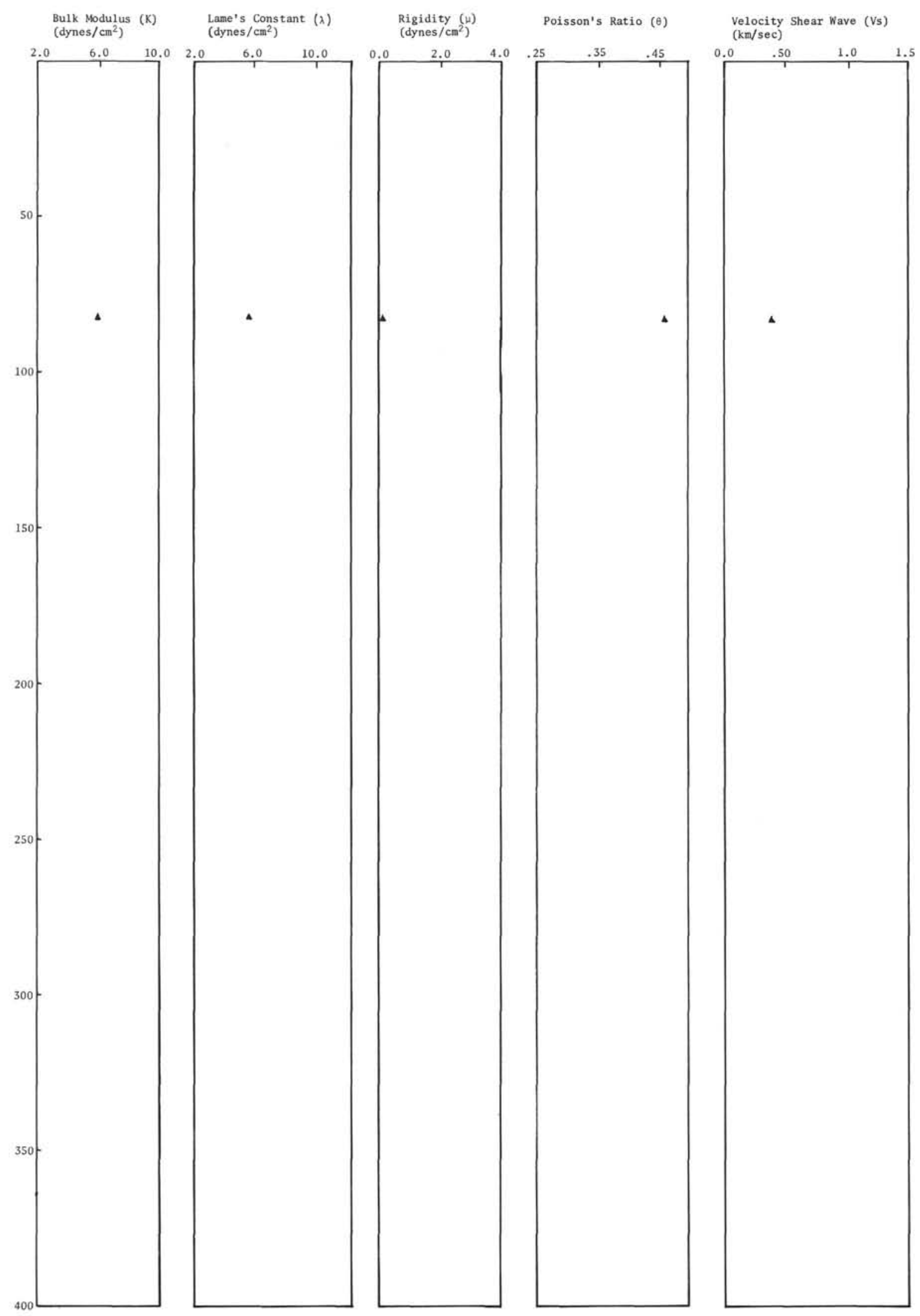

Figure 1. Continued. 


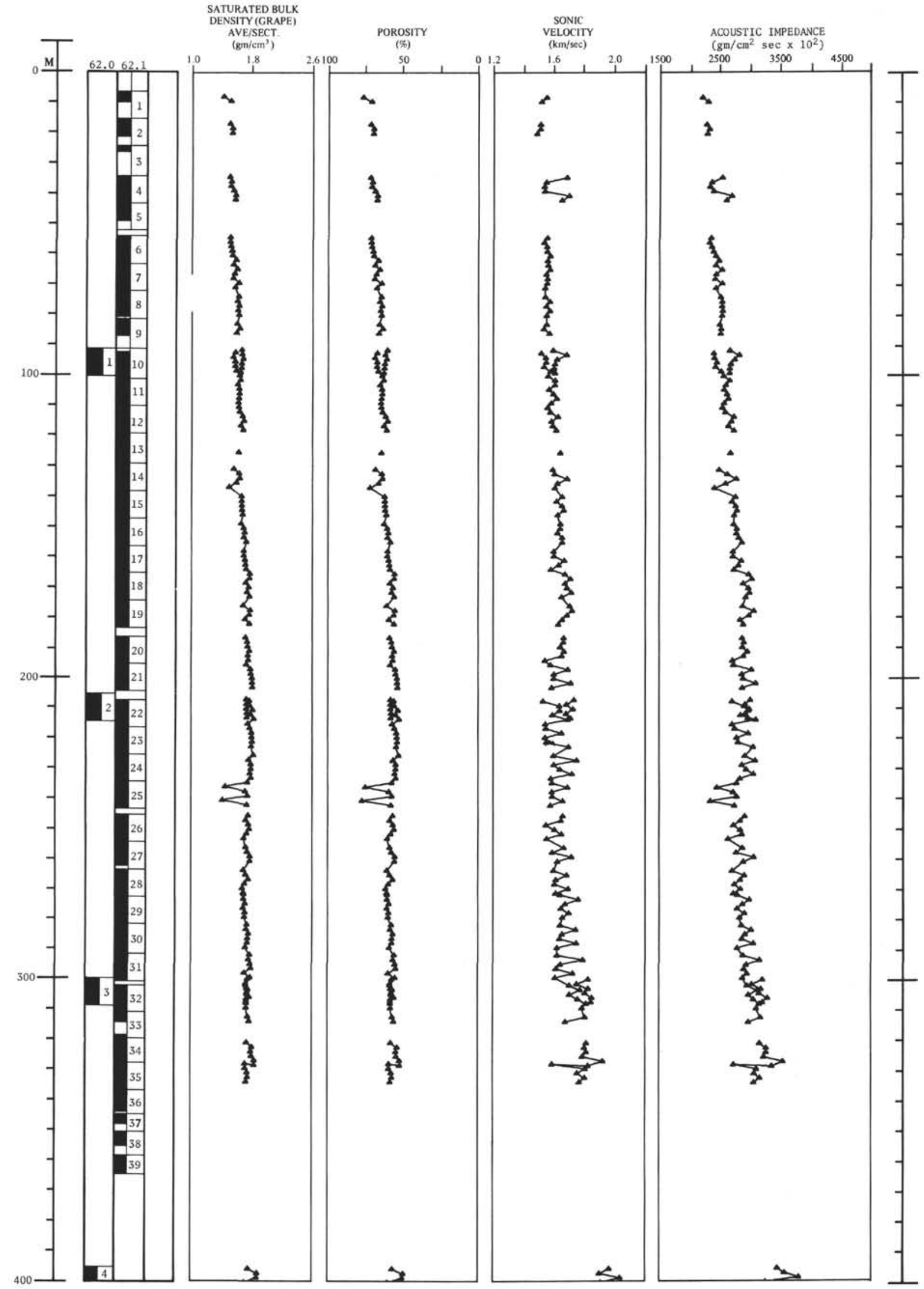

Figure 2. Sound velocity, elastic constants, and related properties, Site 62, 0-400 m. 


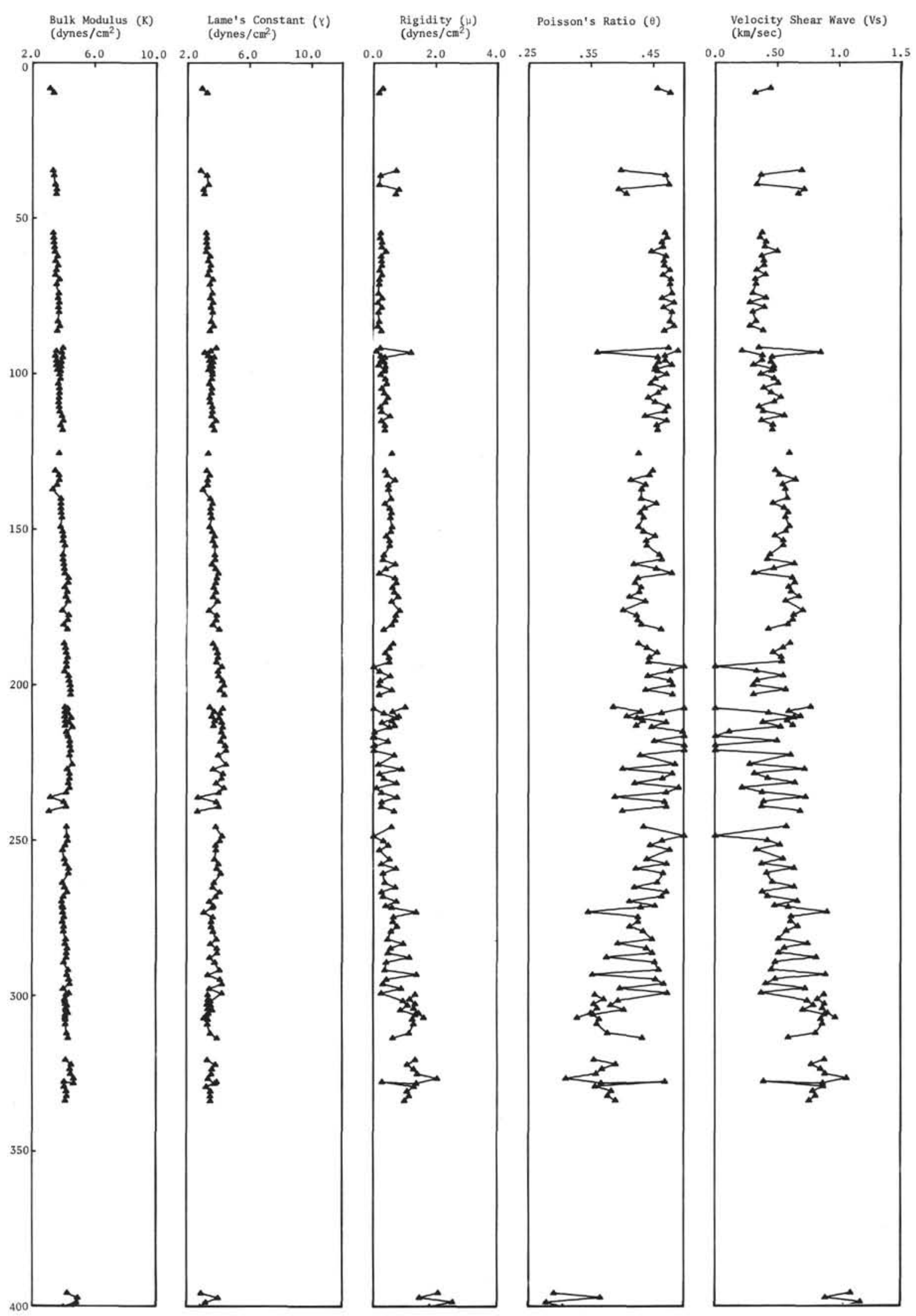

Figure 2. (0-400 m) Continued. 


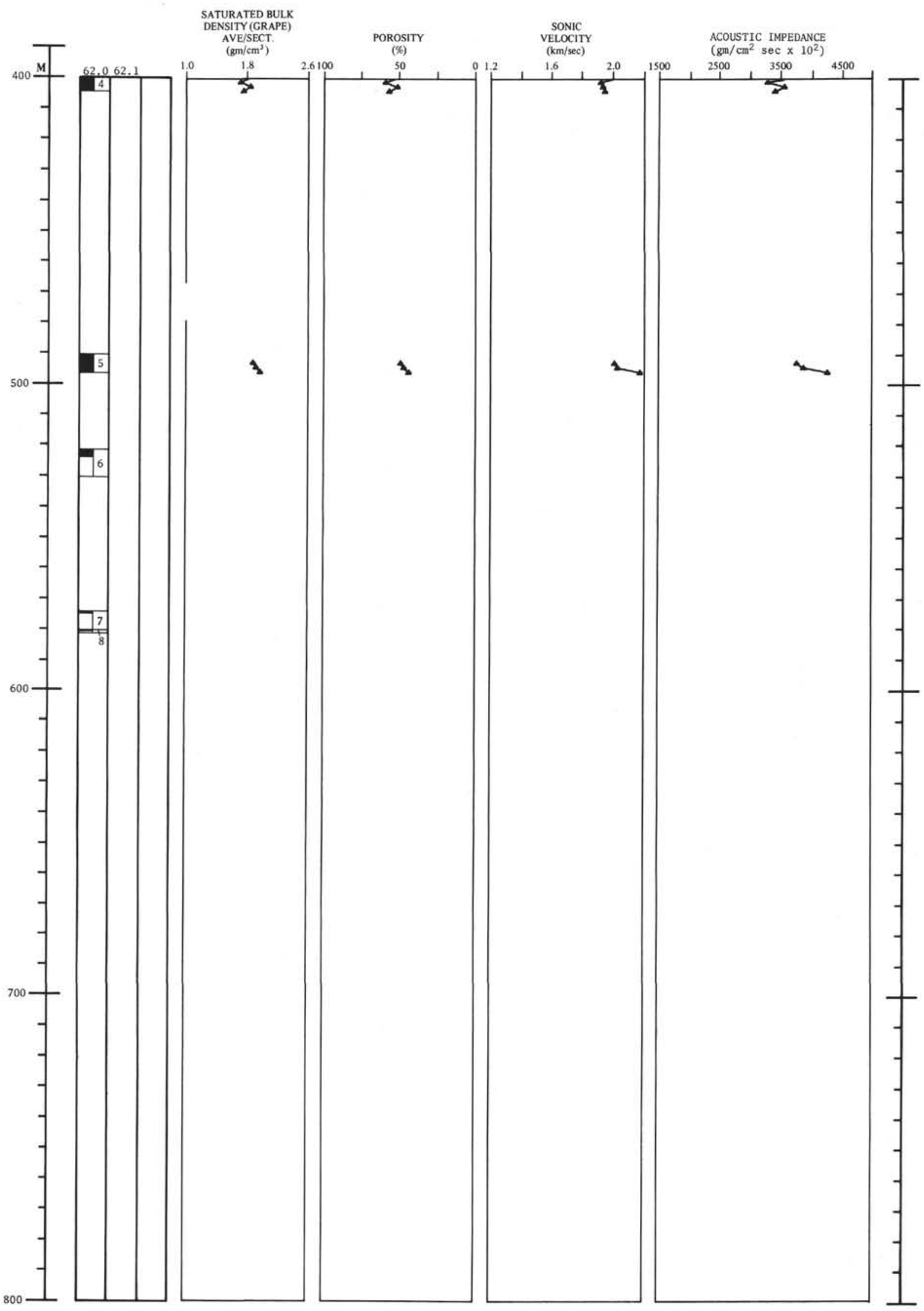

Figure 2. Sound velocity, elastic constants, and related properties, Site 62, 400-800 m. 


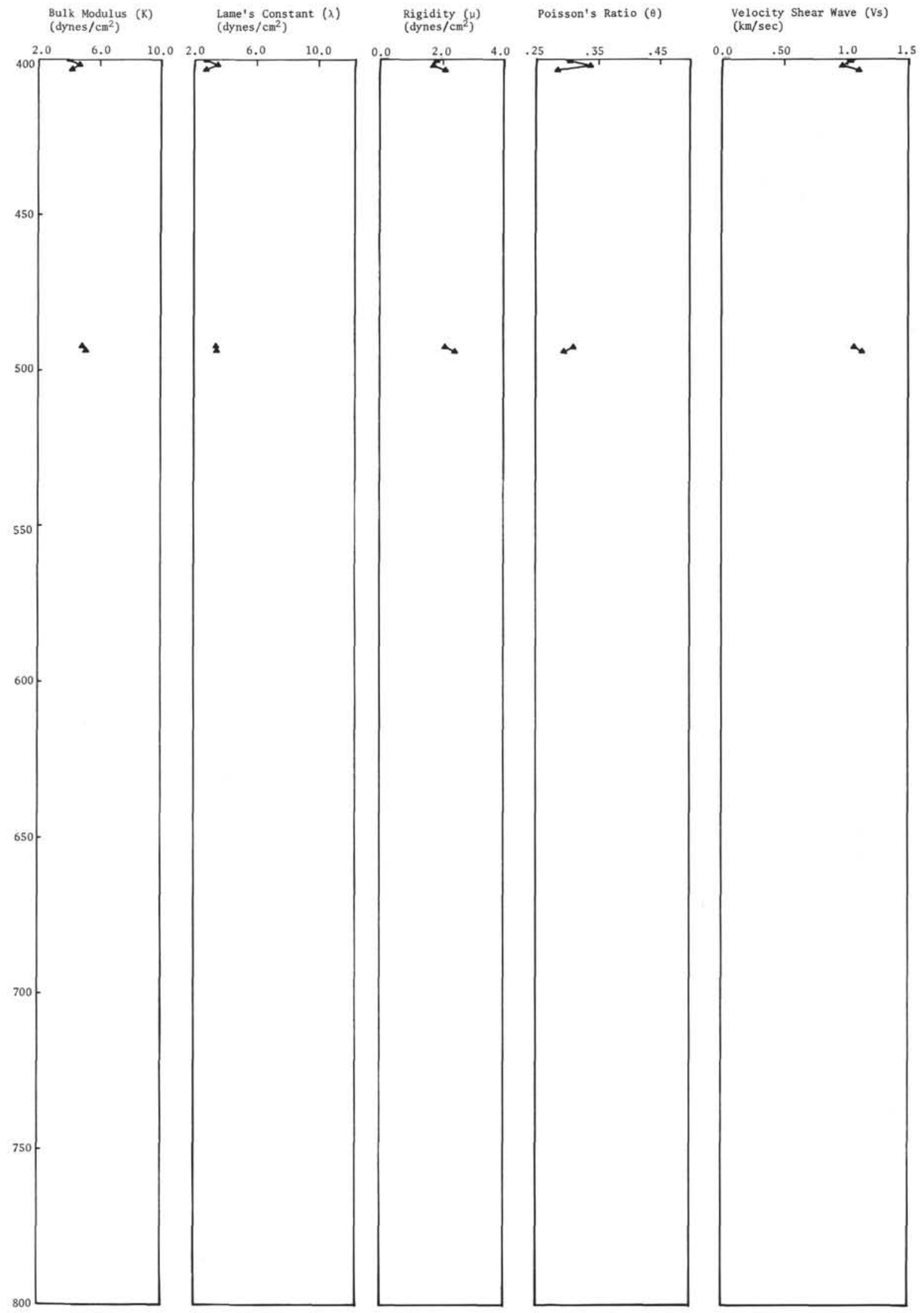

Figure 2. (400-800 m). Continued.

1136 


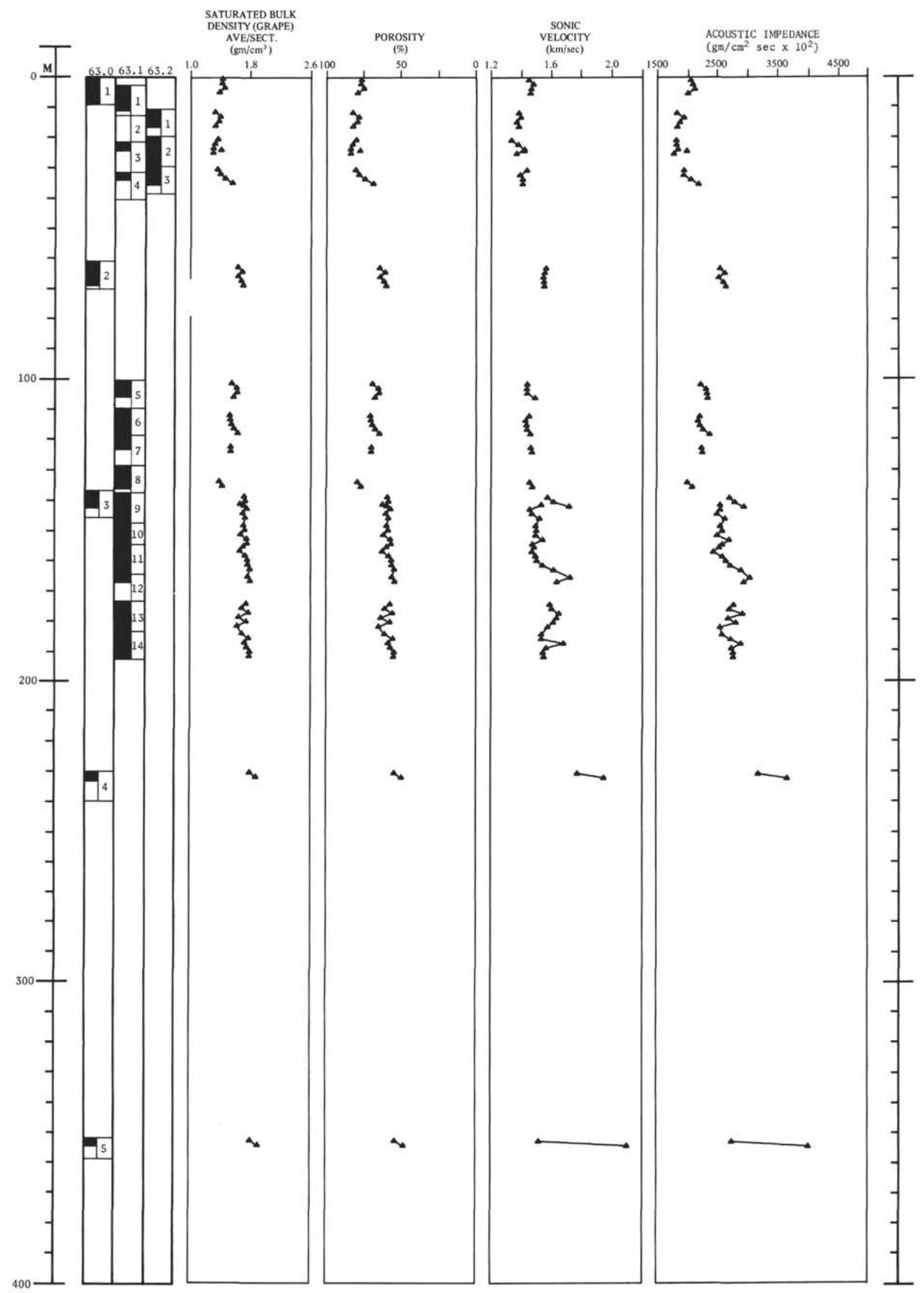

Figure 3. Sound velocity, elastic constants, and related properties, Site 63, 0-400 m. 


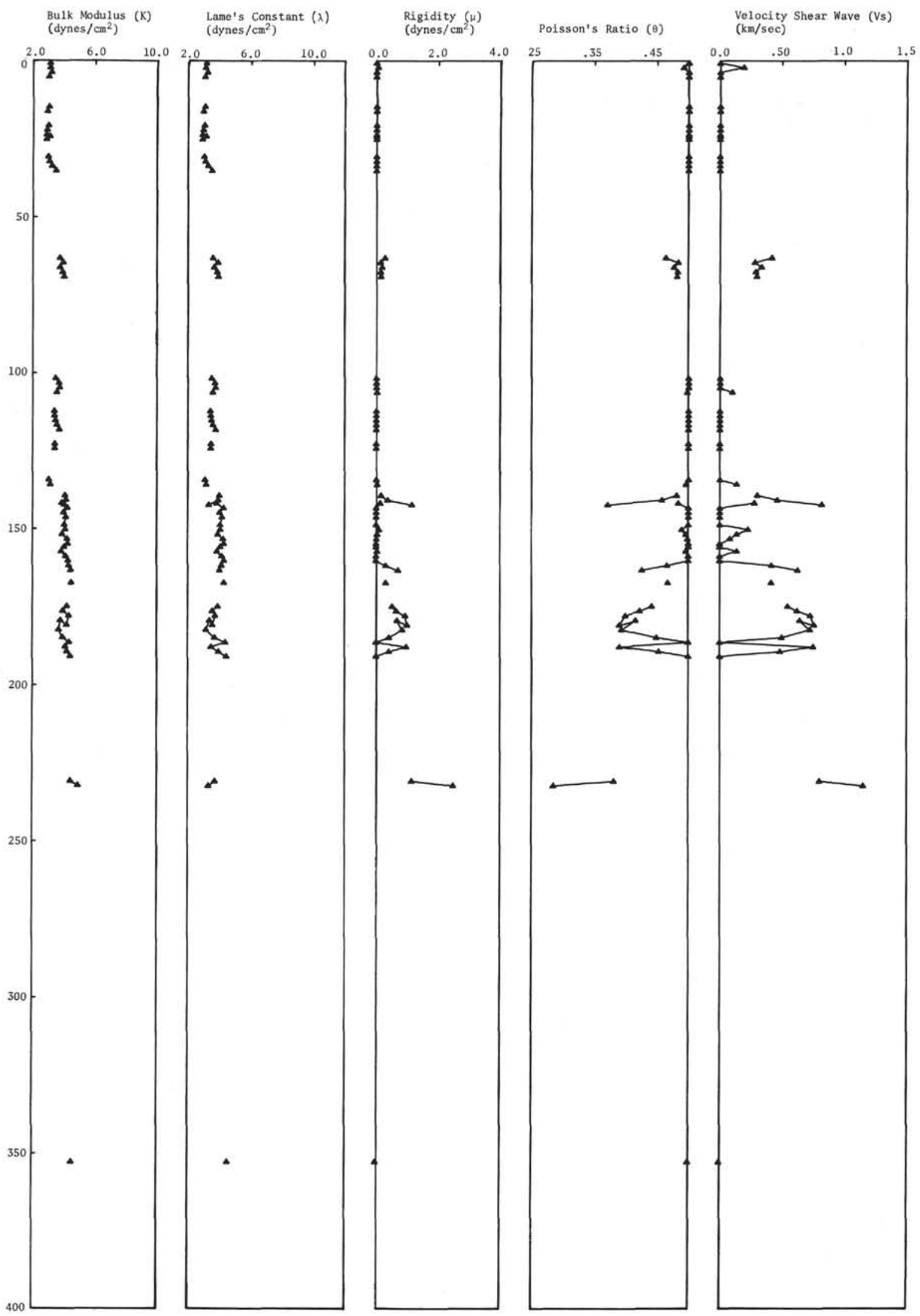

Figure 3. $(0-400 \mathrm{~m})$ Continued. 


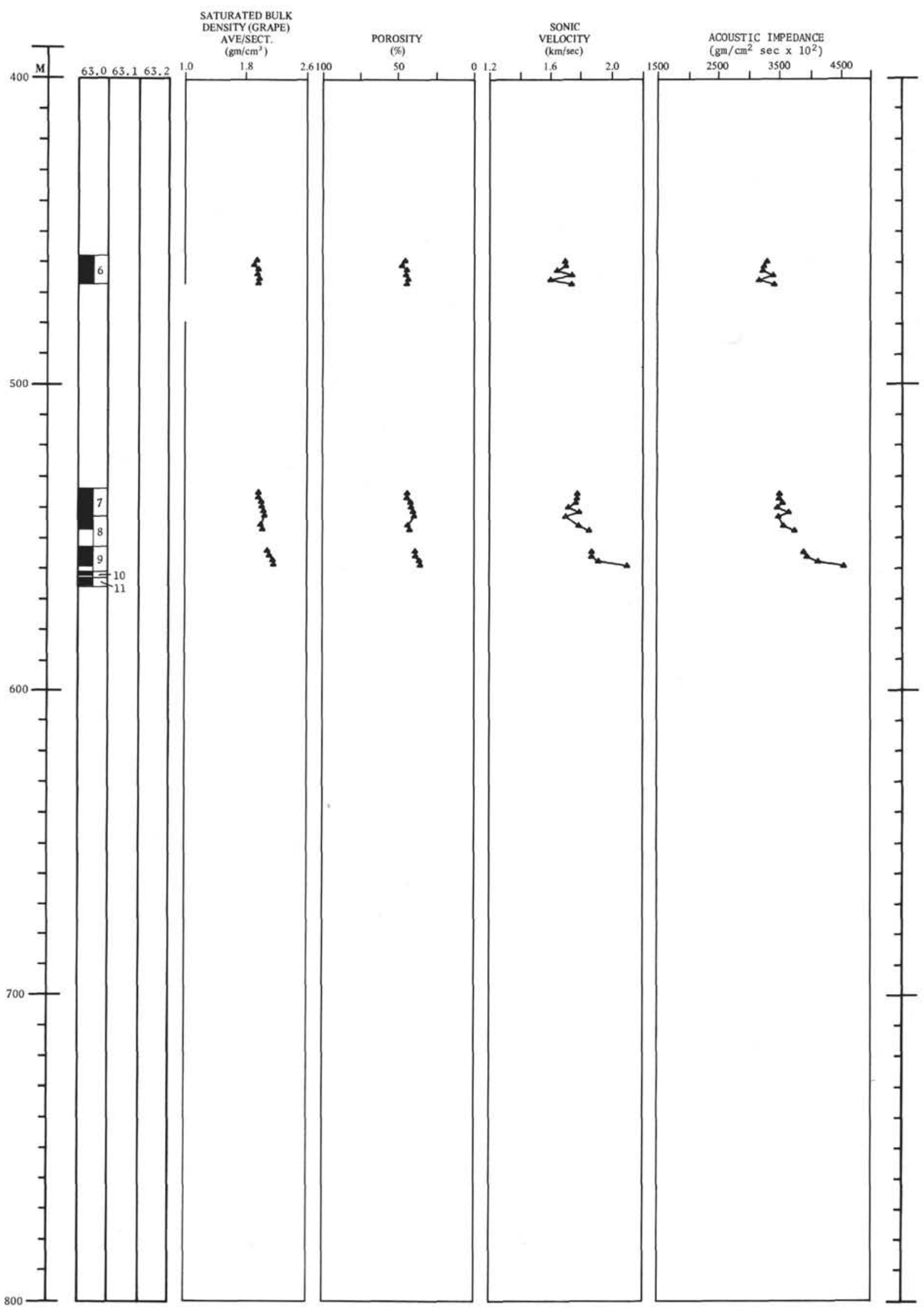

Figure 3. Sound velocity, elastic constants, and related properties, Site $63,400-800 \mathrm{~m}$. 


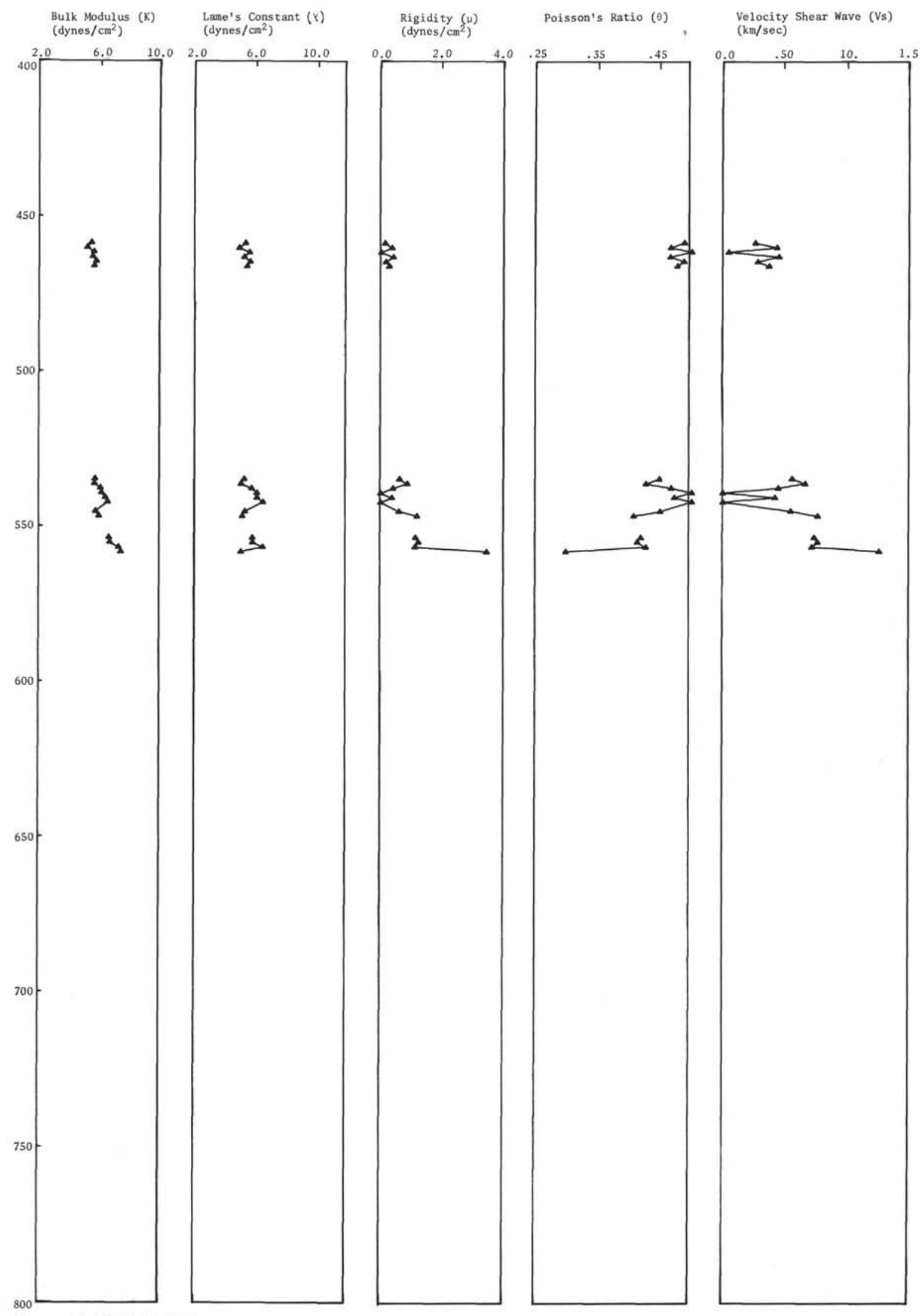

Figure 3. (400-800) Continued. 


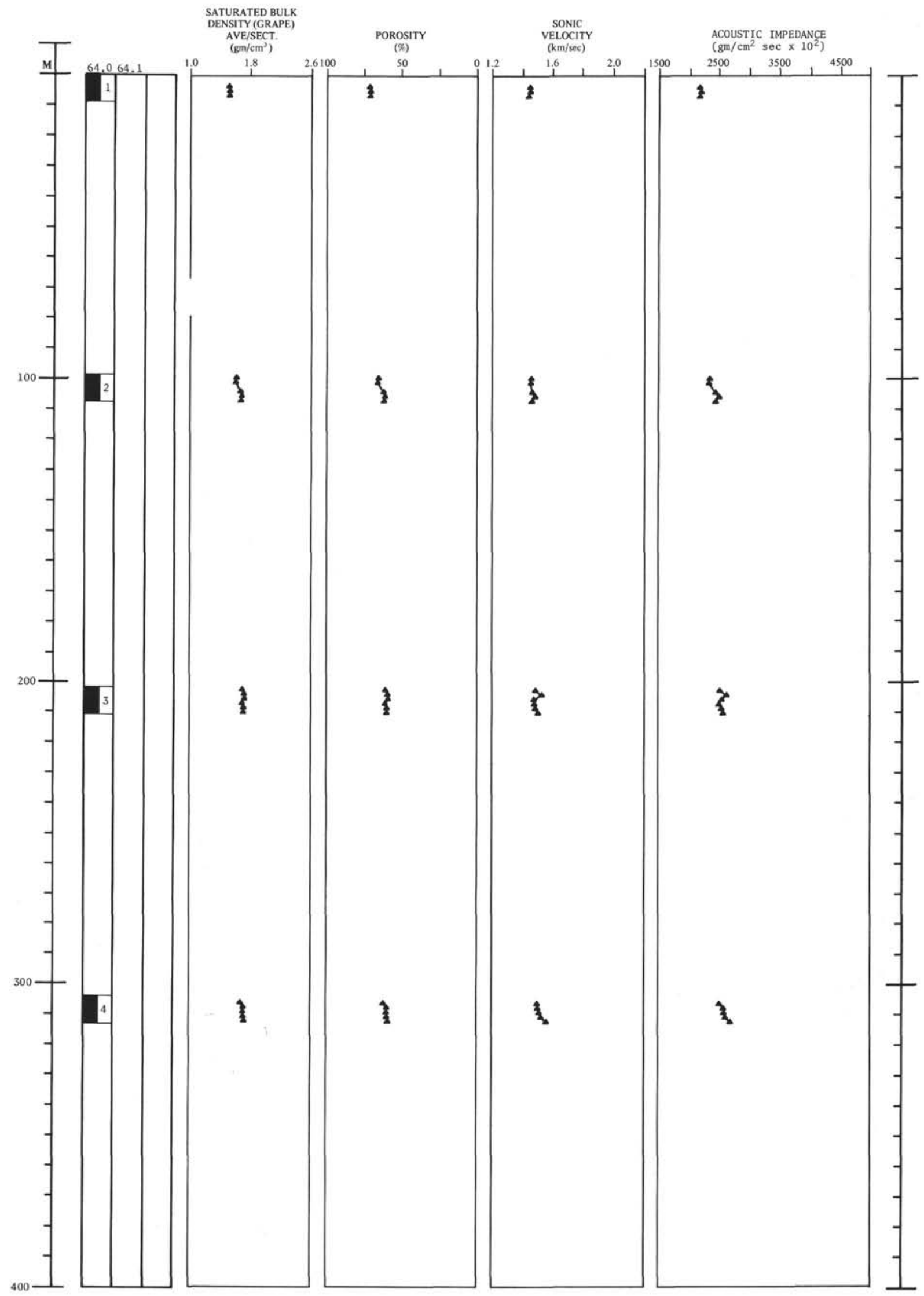

Figure 4. Sound velocity, elastic constants, and related properties, Site 64, 0-400 m. 


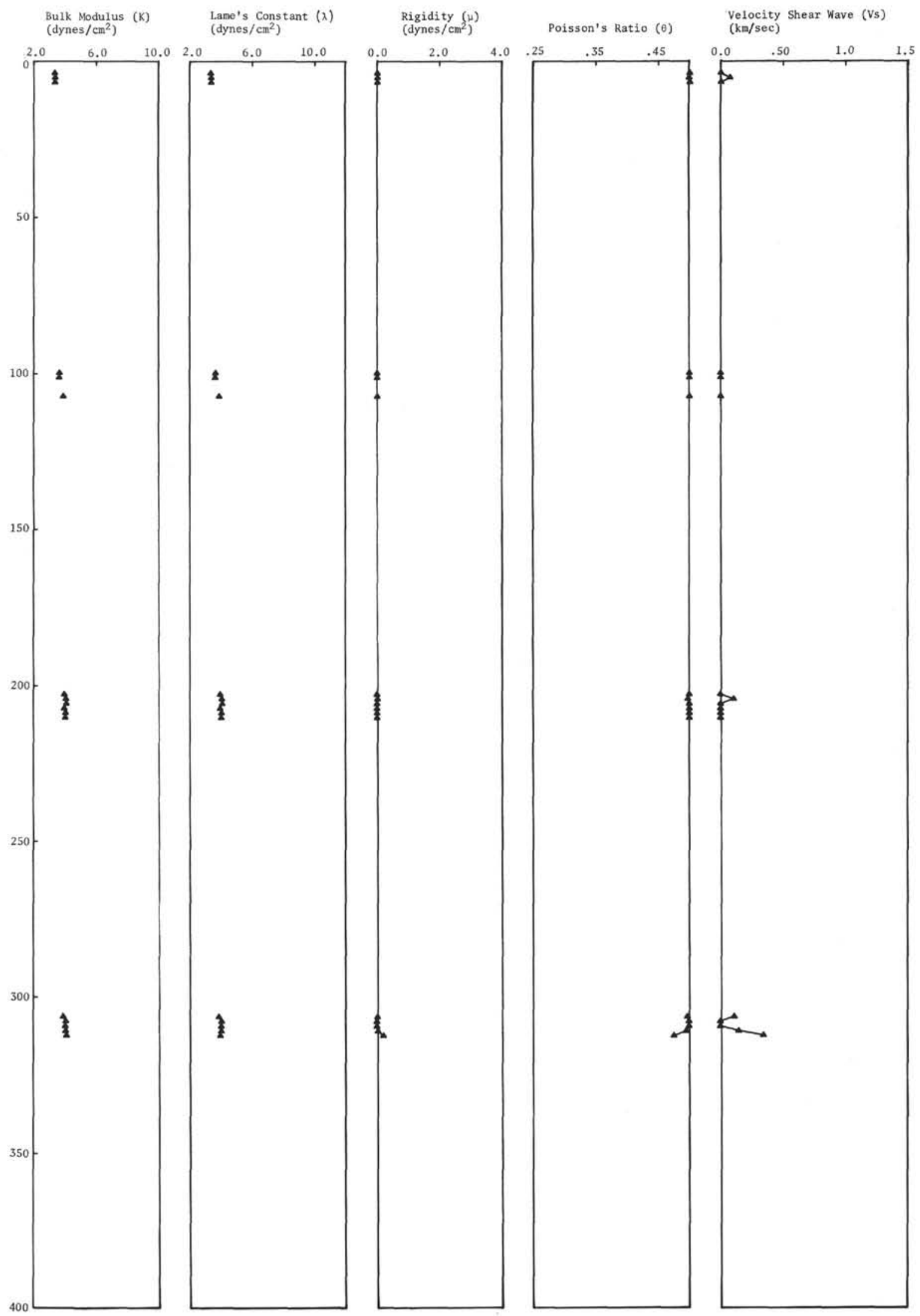

Figure 4. (0-400 m) Continued. 


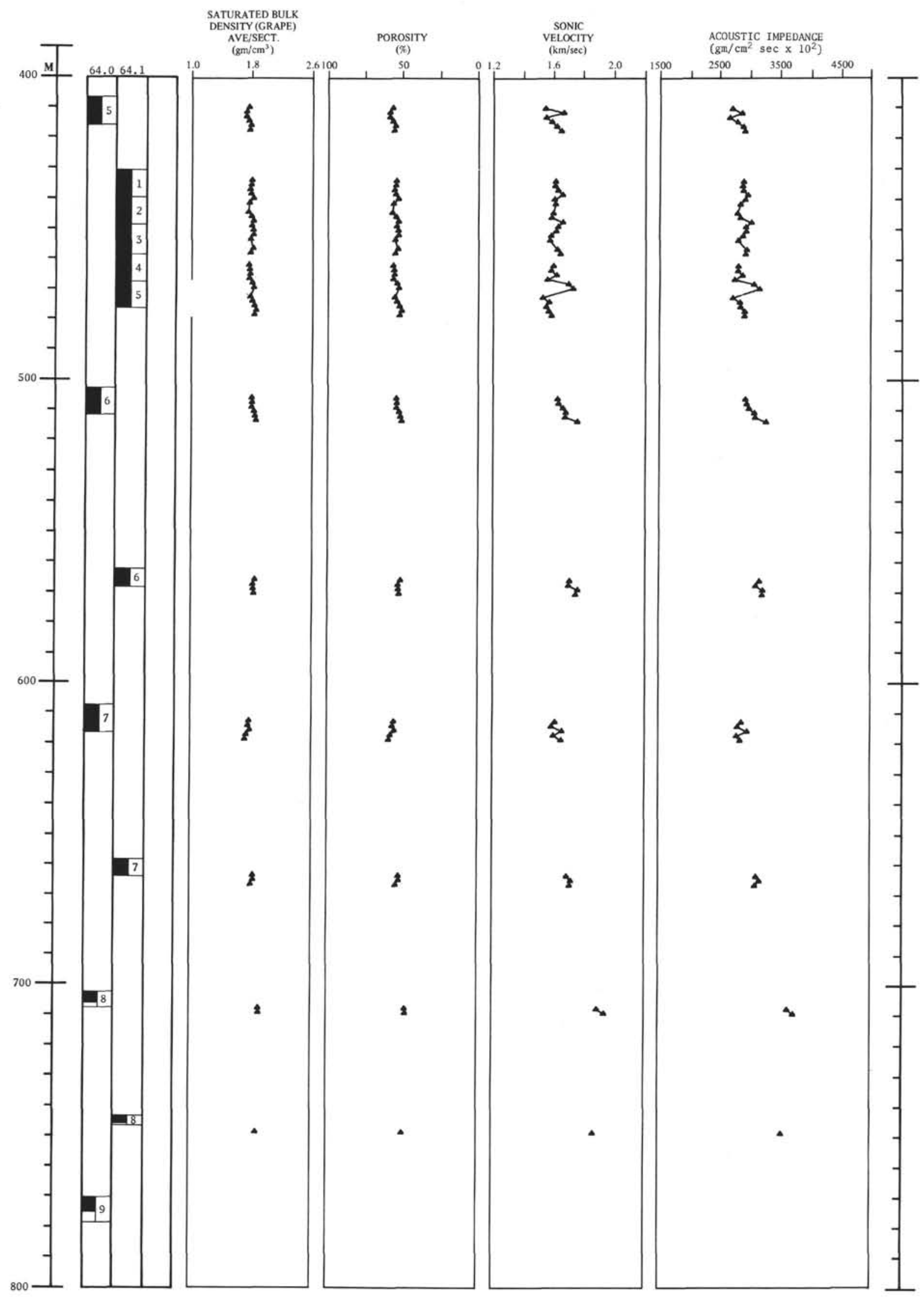

Figure 4. Sound velocity, elastic constants, and related properties, Site 64, 400-800 m. 


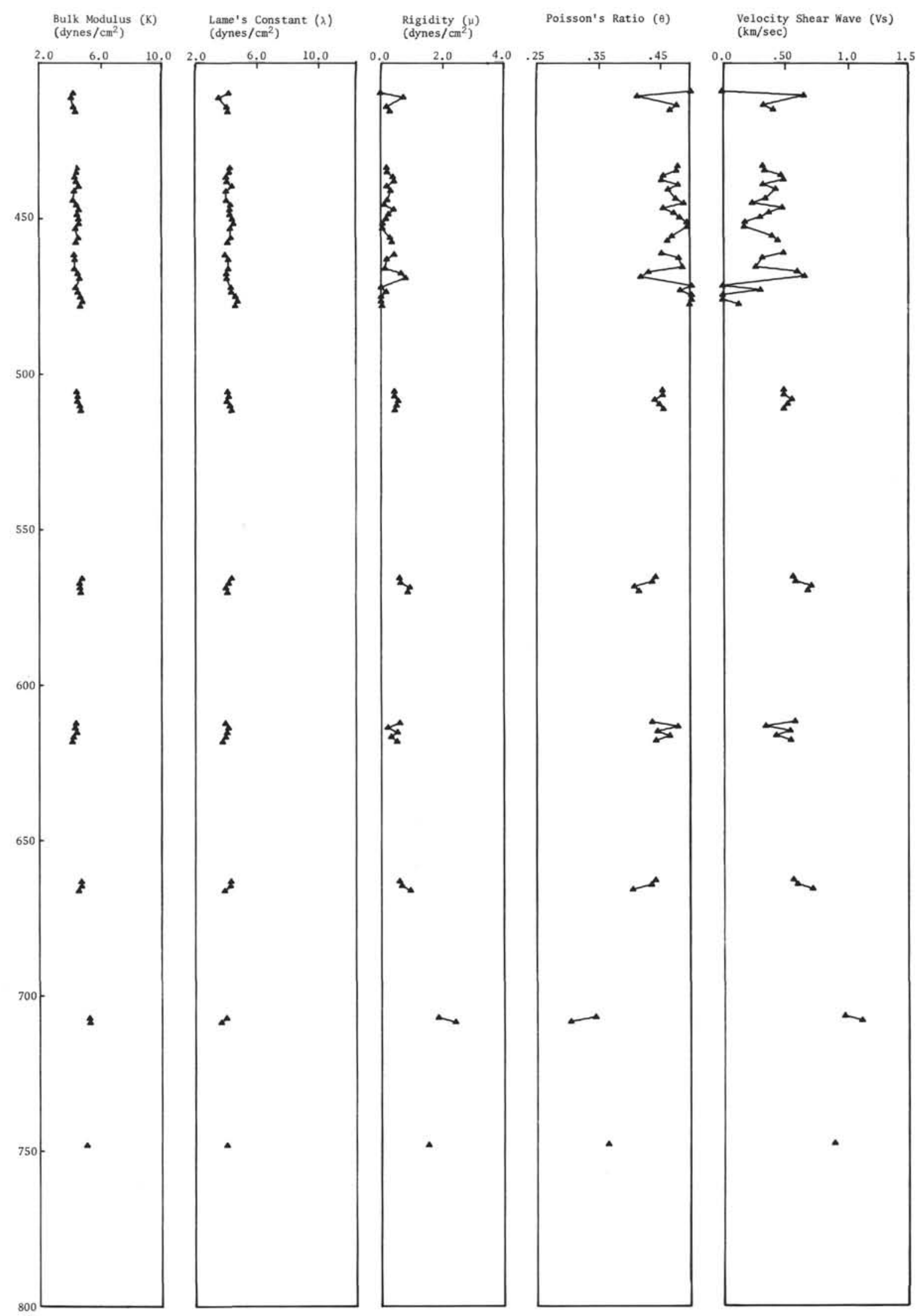

Figure 4. (400-800 m) Continued. 


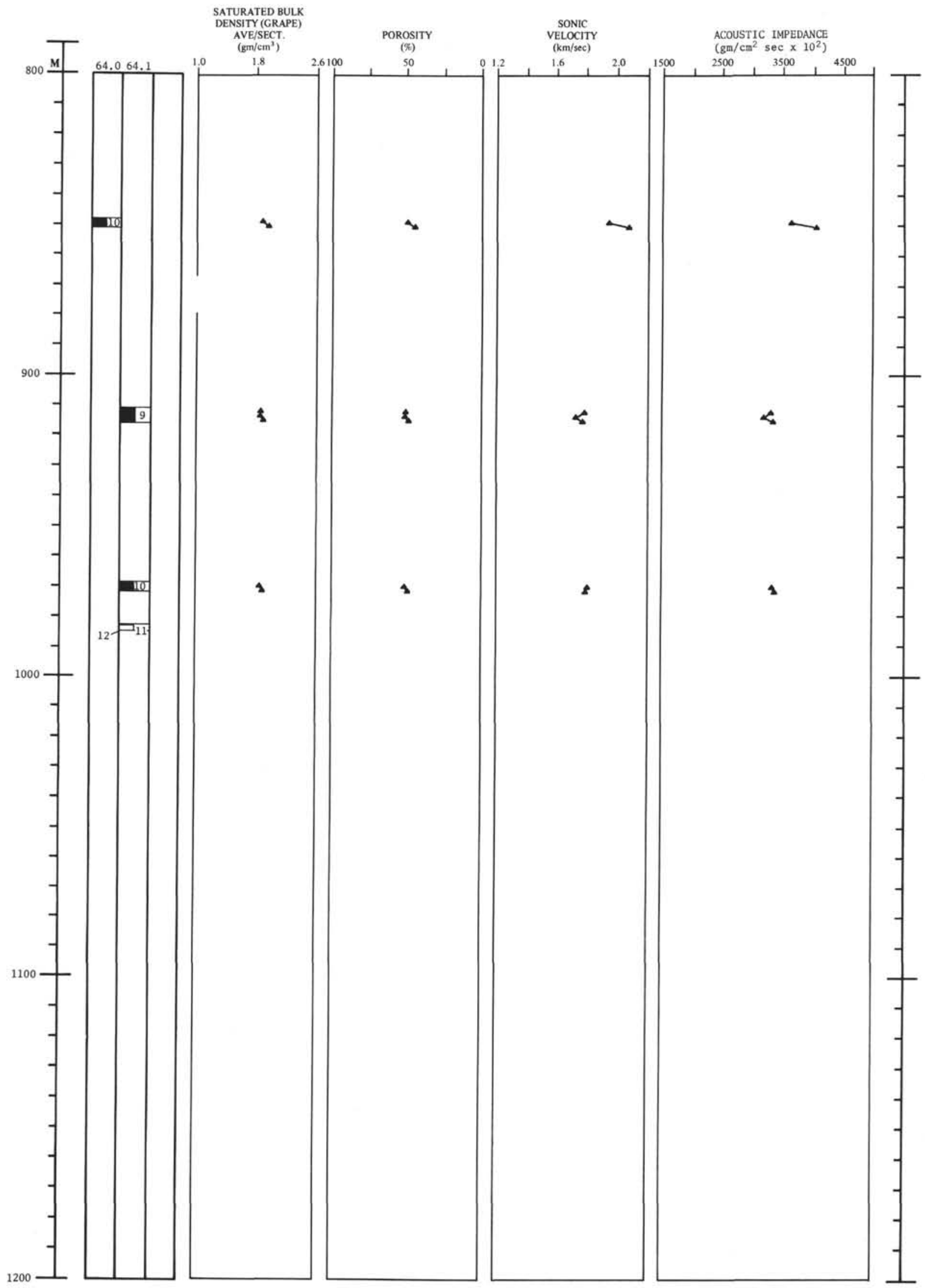

Figure 4. Sound velocity, elastic constants, and related properties, Site 64, 800-1200 m. 


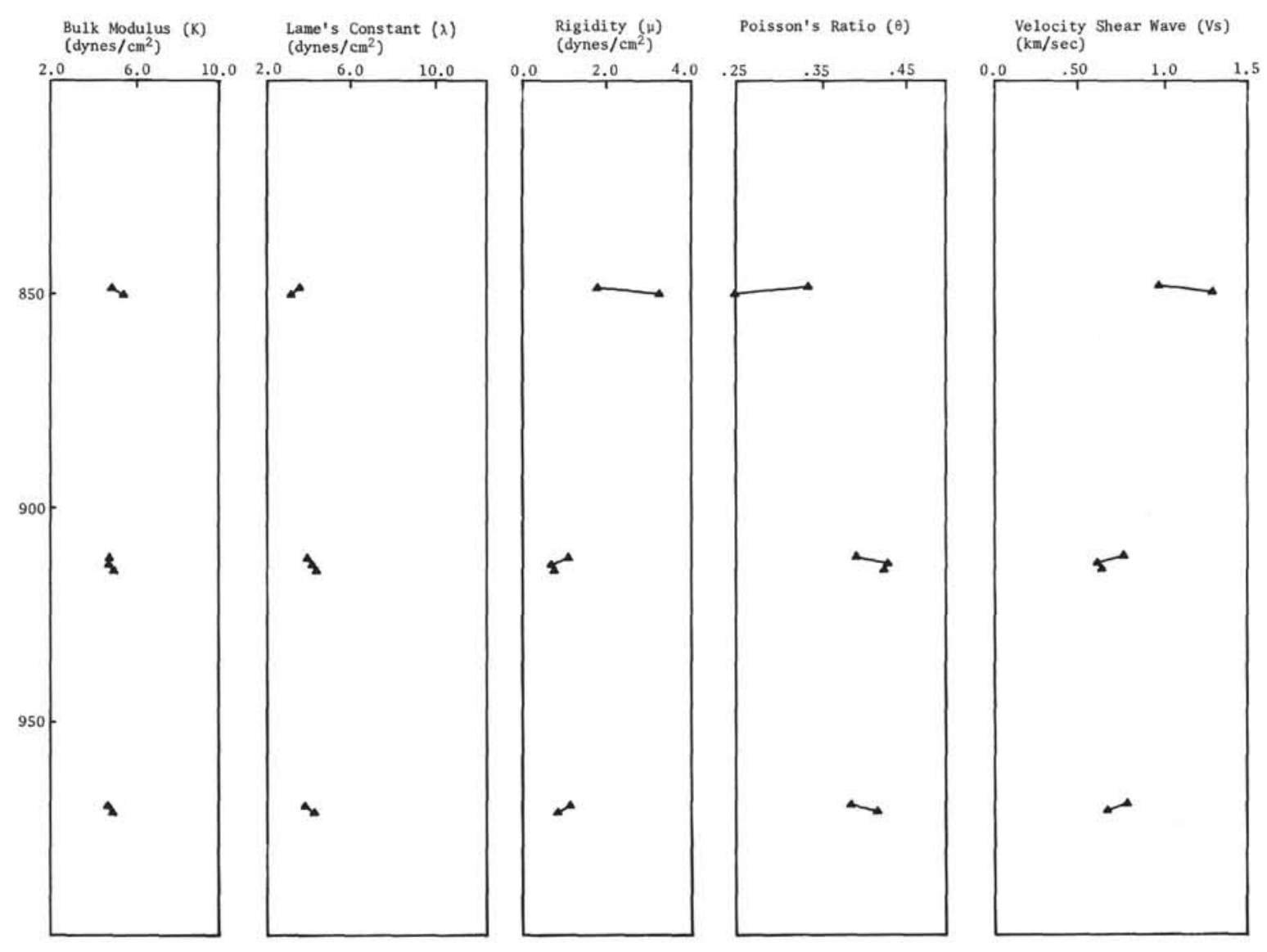

Figure 4. (800-1200) Continued. 


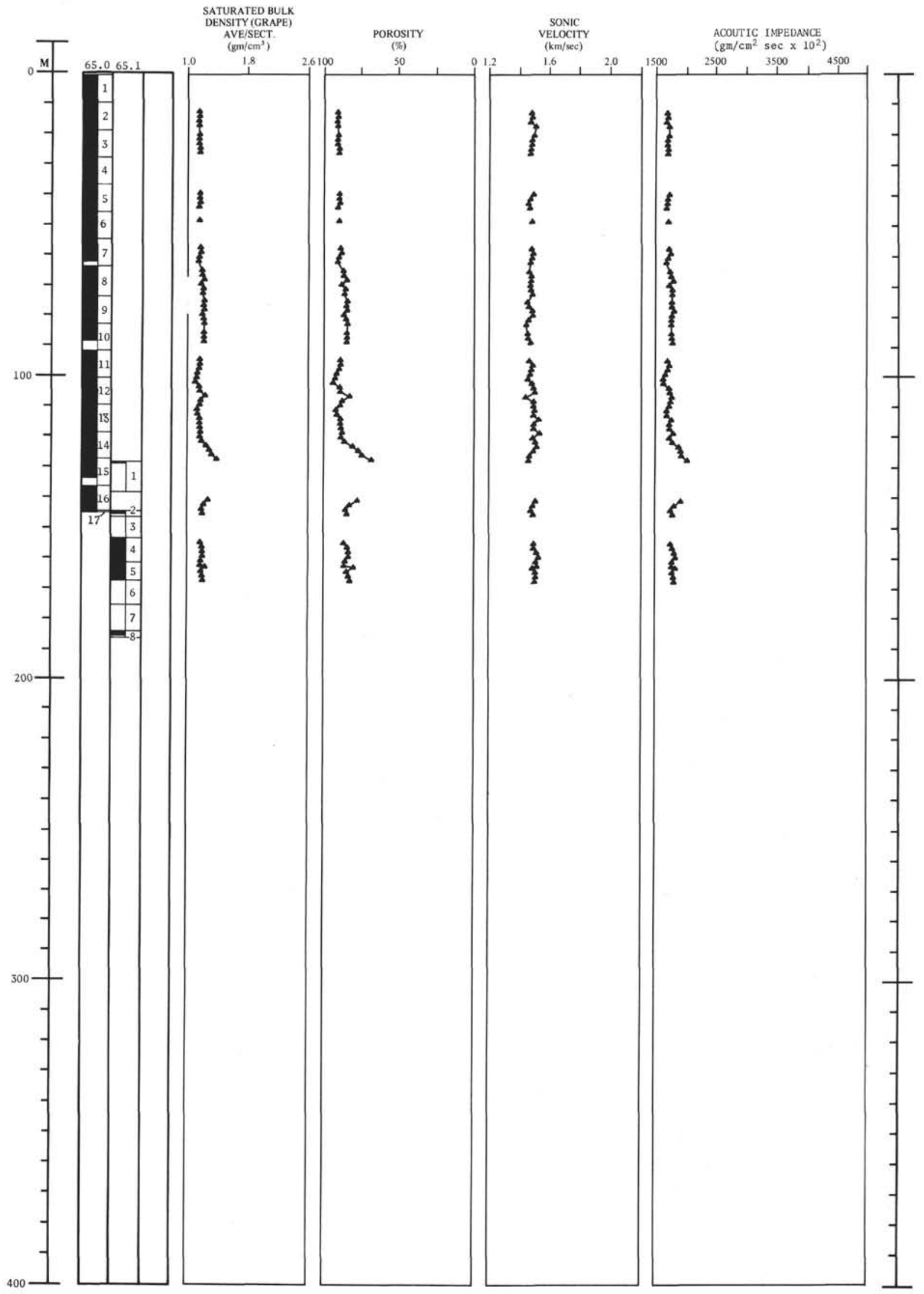

Figure 5. Sound velocity, elastic constants, and related properties, Site 65. 


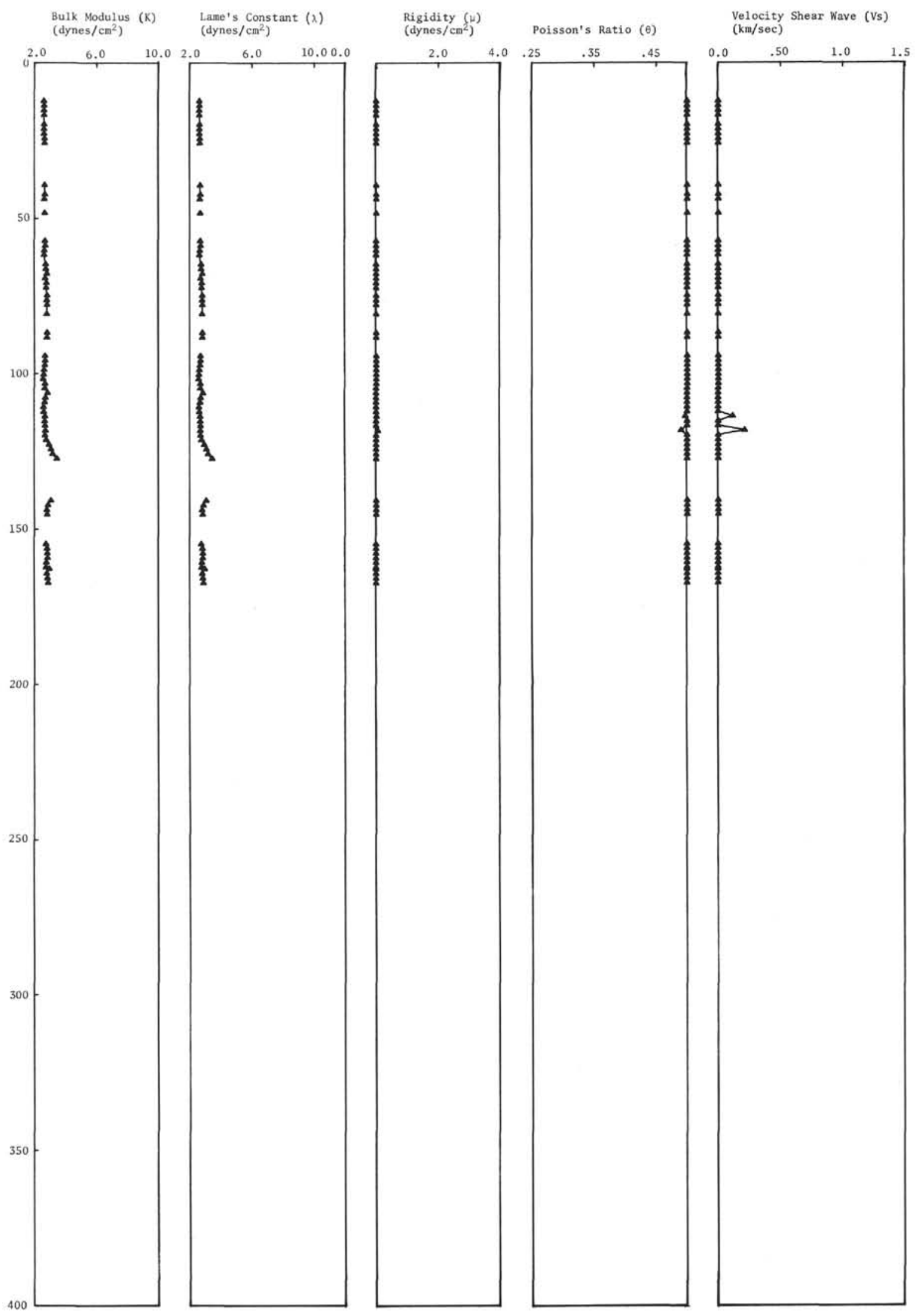

Figure 5. Continued. 


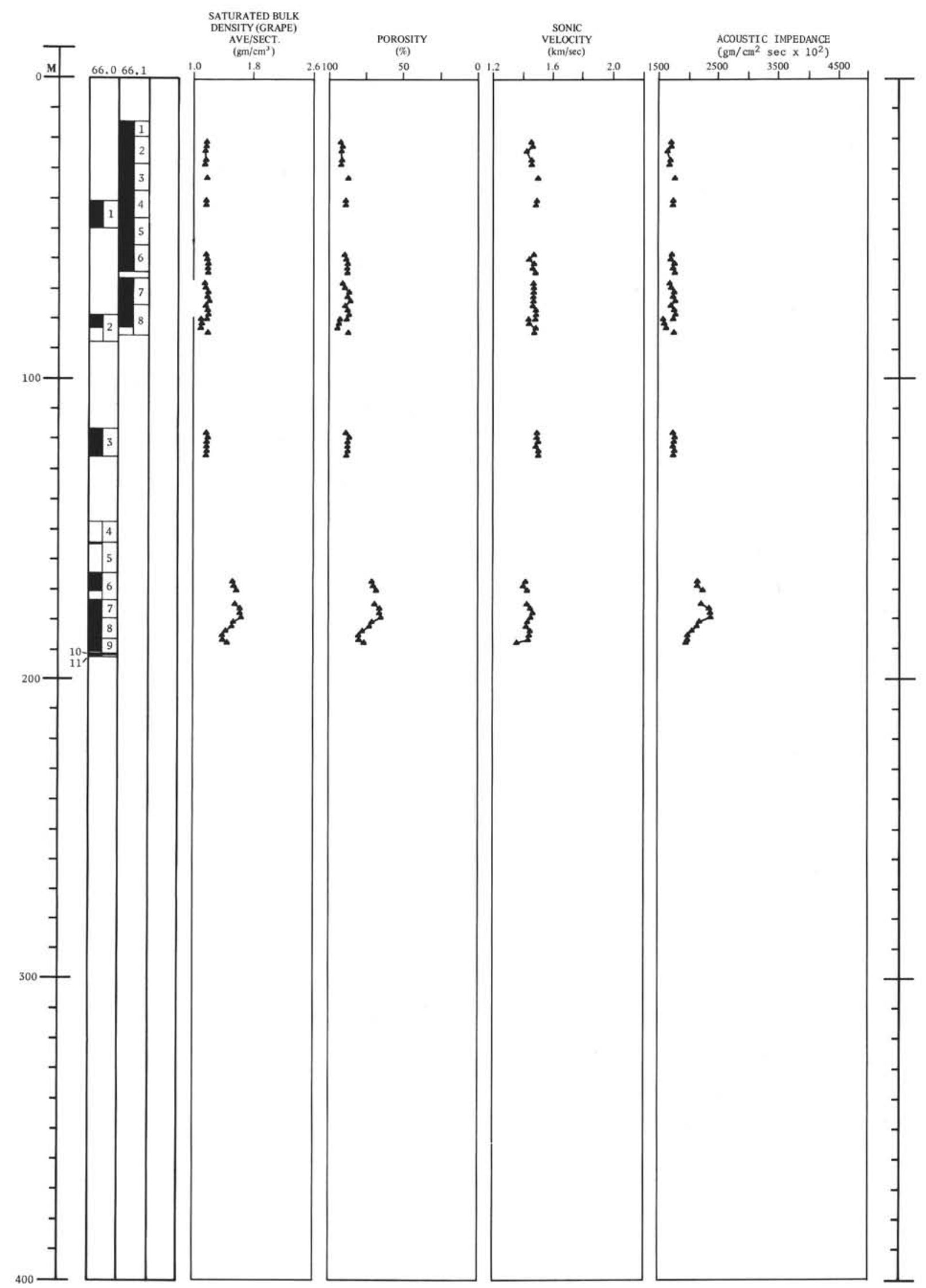

Figure 6. Sound velocity, elastic constants, and related properties, Site 66. 


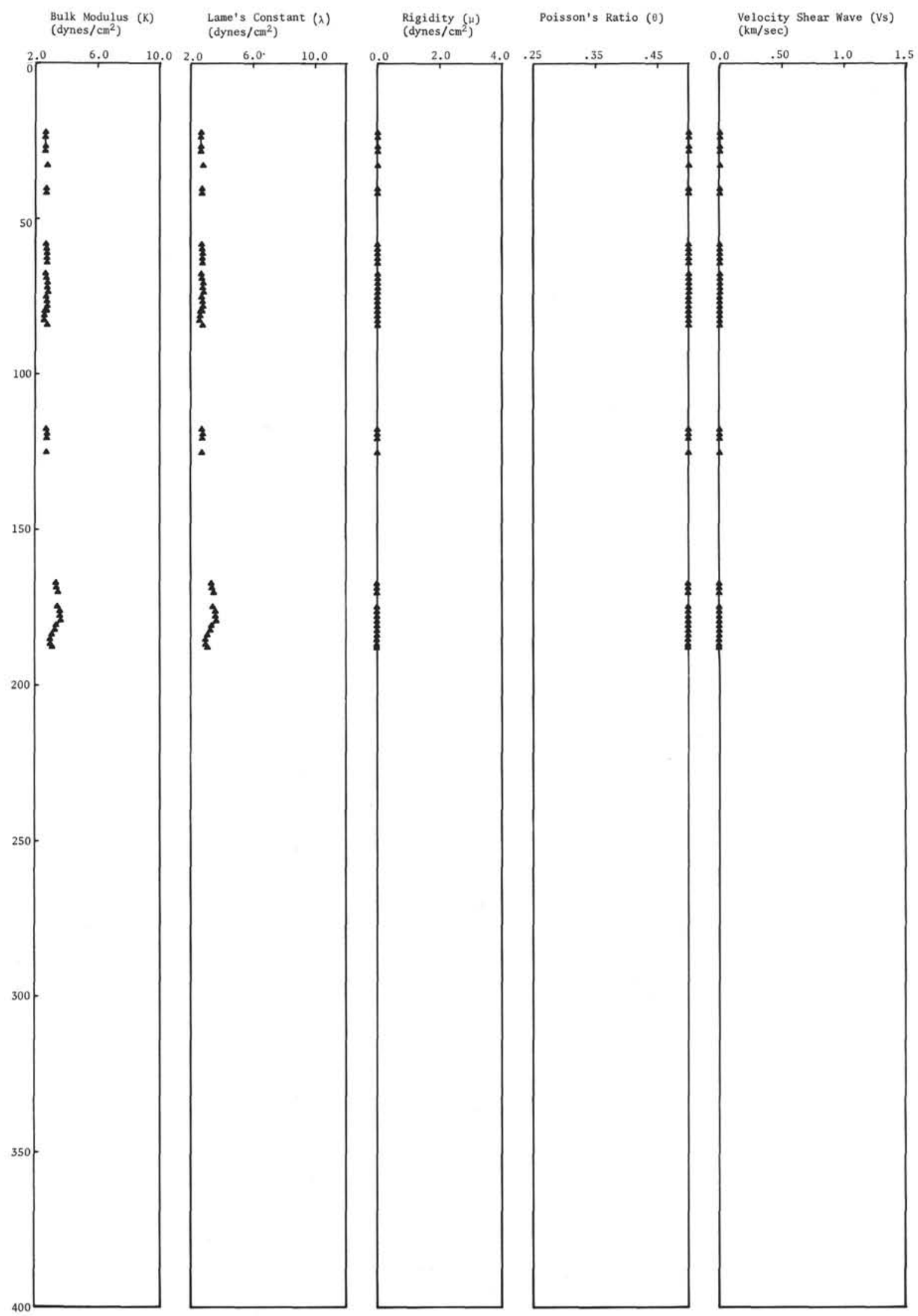

Figure 6. Continued. 
change in shape or volume of the sediment. It is further assumed that such saturated sediments are elastic bodies, the behavior of which can be described by Hookean equations of elasticity. The validity of this approach is discussed by Hamilton (1971).

In Hamilton's (1971) analysis of Gassmann's (1951) work, the velocity of the compressional wave $\left(\mathrm{V}_{\mathrm{p}}\right)$ in sediments is shown to be related to other elastic constants in the following manner:

Velocity compressional wave:

$$
\mathrm{V}_{\mathrm{p}} \times 10^{-5}=\sqrt{\frac{\mathrm{K}+\frac{4}{3} \mu}{\rho \mathrm{B}}}
$$

where: $V_{p}=$ velocity of compressional wave, in kilometers per second,

$\mathrm{K}=$ incompressibility of the total saturated sediment in dynes per square centimeter (the bulk modulus),

$\mu=$ rigidity modulus in dynes per square centimeter, $\begin{aligned} \rho_{\mathrm{B}}= & \text { saturated bulk density in grams per } \\ & \text { cubic centimeter (above). }\end{aligned}$

\section{Acoustic Impedance}

Acoustic Impedance (I) was calculated for all core sections from Sites $62,63,64,65$ and 66 where data were available:

$$
\mathrm{I}=\mathrm{V}_{\mathrm{p}} \cdot \rho_{\mathrm{B}}\left(\mathrm{gm} / \mathrm{cm}^{2} \sec . \times 10^{2}\right)
$$

These values are listed in Tables 2 through 6 and are shown plotted as a function of depth on Figures 2 through 6.

\section{Bulk Modulus}

The bulk modulus $(\mathrm{K})$ of the total saturated sediment was derived for each section recovered from Sites 62, $63,64,65$ and 66 :

$$
\begin{gathered}
\mathrm{K}=\mathrm{K}_{\mathrm{G}}\left(\frac{\mathrm{K}_{\mathrm{S}}+\mathrm{Q}}{\mathrm{K}_{\mathrm{G}}+\mathrm{Q}}\right) \text { in dynes } / \mathrm{cm}^{2} \times 10^{10} \\
{[\text { after Gassmann, (1951)] }}
\end{gathered}
$$

where: $\mathrm{K}_{\mathrm{G}}=$ weighted mean bulk modulus of the solid components, in dynes $/ \mathrm{cm}^{2} \times$ $10^{10}$,

$\mathrm{K}_{\mathrm{S}}=$ skeletal bulk modulus of Gassmann (or the frame bulk modulus of Hamilton), in dynes $/ \mathrm{cm}^{2} \times 10^{10}$, and

$$
\begin{aligned}
\mathrm{Q} & =\frac{\mathrm{K}_{\mathrm{F}}\left(\mathrm{K}_{\mathrm{G}}-\mathrm{K}_{\mathrm{S}}\right)}{\phi\left(\mathrm{K}_{\mathrm{G}}-\mathrm{K}_{\mathrm{F}}\right)} \\
\mathrm{K}_{\mathrm{F}}= & \begin{array}{l}
\text { bulk modulus of the interstitial water, } \\
\text { in dynes/cm } 2 \times 10^{10}
\end{array}
\end{aligned}
$$

The skeletal bulk modulus $\left(\mathrm{K}_{\mathrm{S}}\right)$ can be derived provided that the velocities of both shear wave and compressional wave in the sediments are known. No measurements of shear wave velocities were made on retrieved cores on Leg 7. In the absence of such measurements, the following relationships were used:

For cores from Sites 65 and 66, which consist largely of radiolarian ooze and pelagic clay, a relationship derived by Hamilton (1971) for silt clay was used:

$$
\log \mathrm{K}_{\mathrm{S}}=3.73580-4.25075 \phi,
$$

$$
\text { (where } \mathrm{K}_{\mathrm{S}} \text { is in dynes } / \mathrm{cm}^{2} \times 10^{8} \text { ). }
$$

For cores from Sites 62, 63 and 64, which consist largely of nannofossil chalk ooze, a relationship derived by E. L. Hamilton (personal communication) for calcareous material was used:

$$
\begin{aligned}
& \log \mathrm{K}_{\mathrm{S}}=3.86297-4.05522 \phi, \\
& \text { (where } \mathrm{K}_{\mathrm{S}} \text { is in dynes } / \mathrm{cm}^{2} \times 10^{8} \text { ). }
\end{aligned}
$$

The bulk modulus of the interstitial fluid $\left(\mathrm{K}_{\mathrm{F}}\right)$ is assumed to be: (after NAVOCEANO $\mathrm{S}_{\mathrm{p}}-68,1966$ )

$$
\mathrm{K}_{\mathrm{F}}=2.397082 \times 10^{10} \text { dynes } / \mathrm{cm}^{2} \times 10^{10} .
$$

The bulk modulus of the mineral grains $\left(\mathrm{K}_{\mathrm{G}}\right)$ was approximated as follows:

For cores of radiolarian ooze from Sites 65 and 66, a value derived by E. L. Hamilton (personal communication) for the bulk modulus of opal $\left[\mathrm{K}_{\mathrm{G}(1)}\right]$ was used:

$$
\mathrm{K}_{\mathrm{G}(1)}=34.6 \times 10^{10} \text { dynes } / \mathrm{cm}^{2} \times 10^{10} .
$$

For calcite, a value derived by Peselnick (1962) for the bulk modulus of $\left[\mathrm{K}_{\mathrm{G}(2)}\right]$ is used:

$$
\mathrm{K}_{\mathrm{G}(2)}=72.90 \times 10^{10} \text { dynes } / \mathrm{cm}^{2} \times 10^{10} \text {. }
$$

For clay, a value used by Hamilton (1971) from (Skempton, 1961) for the bulk modulus of clay $\left[\mathrm{K}_{\mathrm{G}(3)}\right]$ is:

$$
\mathrm{K}_{\mathrm{G}(3)}=\left[50.0 \times 10^{10}\right] \text { dynes } / \mathrm{cm}^{2} \times 10^{10} .
$$


For cores from Sites 62, 63, and 64, a weighted value of grain bulk modulus $\left[\mathrm{K}_{\mathrm{G}(4)}\right]$ is used:

$$
\begin{gathered}
\mathrm{K}_{\mathrm{G}(4)}=\left[\mathrm{C} \cdot \mathrm{K}_{\mathrm{G}(2)}+(1-\mathrm{C}) \mathrm{K}_{\mathrm{G}(3)}\right] \\
\text { dynes } / \mathrm{cm}^{2} \times 10^{10}
\end{gathered}
$$

where: $\mathrm{C}=$ fraction of calcium carbonate in solid component of sediment by weight. Gealy (1971b).

For cores of pelagic clay from Site $66, \mathrm{~K}_{\mathrm{G}(3)}$ was used for the grain bulk modulus.

Values of $\mathrm{K}$ are listed in Tables 2 through 6 and are shown plotted as a function of depth on Figures 2 through 6 . These values of $\mathrm{K}$ were then used with measured values of density and velocity to compute other elastic moduli.

\section{Rigidity}

The rigidity $(\mu)$ of the total saturated sediment was derived for each core section recovered from Sites 62, $63,64,65$ and 66 where data were available.

$$
\begin{gathered}
\mu=3 / 4\left[\rho_{\mathrm{B}}\left(\mathrm{V}_{\mathrm{p}}\right)^{2}-\mathrm{K}\right] \text { dynes } / \mathrm{cm}^{2} \times 10^{10} \\
\text { (after Hamilton, 1971) }
\end{gathered}
$$

Values of $\mu$ are listed in Tables 2 through 6 and are shown plotted as a function of depth on Figures 2 through 6 .

\section{Lame's Constant}

Lame's constant $(\lambda)$ of the total saturated sediment was derived for each core section recovered from Sites $62,63,64,65$ and 66 where data were available:

$$
\lambda=\left[\mathrm{K}-\frac{2}{3} \mu\right] \text { dynes } / \mathrm{cm}^{2} \times 10^{10} .
$$

Values derived are listed in Tables 2 through 6 and are shown plotted as a function of depth on Figures 2 through 6 .

\section{Poisson's Ratio}

Poisson's ratio $(\sigma)$ of the total saturated sediment was derived for each core section recovered from Sites 62, $63,64,65$ and 66 where data were available:

$$
\sigma=\frac{3 \mathrm{~K}-\rho_{\mathrm{B}}\left(\mathrm{V}_{\mathrm{p}}\right)^{2}}{3 \mathrm{~K}+\rho_{\mathrm{B}}\left(\mathrm{V}_{\mathrm{P}}\right)^{2}}
$$

Values derived are listed in Tables 2 through 6 and are shown plotted as a function of depth on Figures 2 through 6.

\section{Shear Wave Velocity}

Shear wave velocity $\left(\mathrm{V}_{\mathrm{s}}\right)$ of the total saturated sediment was derived for each core section recovered from Sites 62, 63, 64, 65 and 66 where data were available:

$$
\mathrm{V}_{\mathrm{S}}=\left(\frac{\mu}{\rho_{\mathrm{B}}}\right)^{1 / 2} \times 10^{5} \text {, in km/sec. }
$$

Values derived are listed in Tables 2 through 6 and are shown plotted as a function of depth on Figures 2 through 6 .

\section{RESULTS}

\section{Site 61}

Measurements of the velocity of the compressional wave were made with the shipboard velocimeter on only one section from Site 61 (61.1-1-2). The maximum measurement was $1.78 \mathrm{~km} / \mathrm{sec}$. Despite core disturbances, the sediment has some rigidity and a shear wave velocity of $0.35 \mathrm{~km} / \mathrm{sec}$ is indicated.

\section{Site 62}

Holes at Site 62 penetrated about 500 meters of pure nannofossil chalk ooze. The section penetrated was cored almost continuously from 50 to 350 meters below the sea floor, and other cores above and below this interval give good coverage to a depth of about 500 meters. Measurements of the velocity of the compressional wave were made with the shipboard velocimeter on almost all sections.

The velocity of the compressional wave of one chalk sample from Site 62 was measured at the University of Hawaii. A sample from 62.1-36-5 from 130 to 134 centimeters has a velocity $\left[\mathrm{V}_{\mathrm{p}(\max )}\right]$ of $2.018 \mathrm{~km} / \mathrm{sec}$ when measured perpendicular to the bedding, and $2.109 \mathrm{~km} / \mathrm{sec}$ when measured horizontal to the bedding. This compares with a velocity $\left[\mathrm{V}_{\mathrm{p}(\max .)}\right]$ of $1.8 \mathrm{~km} / \mathrm{sec}$ measured with the shipboard velocimeter in the overlying core, and of about $2.0 \mathrm{~km} / \mathrm{sec}$ in Core 62.0-4 at 400 meters.

The maximum values of the velocity of the compressional wave in cores from Site 62, in general, increase exponentially with depth from a projected value of $1.5 \mathrm{~km} / \mathrm{sec}$ near the surface to about 2.1 $\mathrm{km} / \mathrm{sec}$ at 490 meters. Exceptions to this regular increase occur at two horizons: Several sections near 35 meters below the sea floor show velocities $\left[\mathrm{V}_{\mathrm{p}(\max .)}\right]$ higher than the trend would indicate (1.7 $\mathrm{km} / \mathrm{sec}$ versus 1.52); and, cores from the interval between 230 and 300 meters show velocities $\left[\mathrm{V}_{\mathrm{p}(\max .)]}\right]$ lower than expected by this trend. There is, in fact, a reversal: the maximum velocity 
$\left[\mathrm{V}_{\mathrm{p} \text { (max.) })}\right]$ of sections from 225 meters is about 1.7 $\mathrm{km} / \mathrm{sec}$, whereas the maximum velocity $\left[\mathrm{V}_{\mathrm{p}}(\max ).\right]$ of sections from 250 meters is only $1.6 \mathrm{~km} / \mathrm{sec}$. This decrease is paralleled by a slight decrease in saturated bulk density (from 1.79 to $1.72 \mathrm{gm} / \mathrm{cm}^{3}$ ) and by a decrease in rigidity (from 4.5 to 4.0 dynes $/ \mathrm{cm}^{2}$ ).

The difference between velocity $\left[\mathrm{V}_{\mathrm{p}(\max .)}\right]$ of successive core sections generally is small $( \pm 0.4 \mathrm{~km} / \mathrm{sec})$ to a depth of about 290 meters. Below this depth the variations are greater $( \pm 0.7 \mathrm{~km} / \mathrm{sec})$. It is at about this depth that lithificiation of the chalk increases markedly, and the scatter in rigidity values also increases. Some of these low values of velocity $\left[\mathrm{V}_{\mathrm{p}(\max .)}\right]$ may reflect disturbances of the material by coring with attendant loss of rigidity. However, the semiconsolidated chalk sequence consists of alternating intervals of more and less indurated materials, and these natural variations may account for much of the scatter in the data. If the envelope of the values below 190 meters is considered, the maximum envelope probably represents values of undisturbed lithified sediment most closely; the minimum envelope the unconsolidated and/or disturbed equivalent.

The rigidity and the maximum envelope of derived values of shear wave velocity increase with depth. Also, the scatter in these data increases with depth. Even near surface sediments show some capacity to sustain shear stress. Sediments at 9 meters have a shear wave velocity of 0.3 to $0.4 \mathrm{~km} / \mathrm{sec}$. At 150 meters, the maximum shear wave velocity is about $0.55 \mathrm{~km} / \mathrm{sec}$; at 300 meters it is about $0.8 \mathrm{~km} / \mathrm{sec}$; and at 490 meters it is as high as $1.27 \mathrm{~km} / \mathrm{sec}$. Zero values of rigidity between about 200 and 250 meters probably reflect coring disturbances.

Ratios between the maximum compressional wave velocity and the maximum shear wave velocity as measured on cores $\left[\mathrm{V}_{\mathrm{p}(\max .)} / \mathrm{V}_{\mathrm{s}}\right]$ decrease with depth. At 550 meters in nannofossil ooze, the ratio is 5.0. At 250 meters in partially lithified chalk ooze the ratio is 3.3. At 400 meters in lithified chalk the ratio is 1.9. At 495 meters in soft limestone the ratio is 1.8 . Molotova and Vassil'ev (1960) give data which indicate that these ratios range from about 1.8 to 3.5 in chalks and from about 1.5 to 1.95 in limestones; Press (1966) shows that saturated clays have ratios which range from 2.08 to 13.7 . Thus, it appears likely that derived values of shear wave velocity at Site 62 are valid, provided that maximum values are used. Very low values without doubt reflect core disturbance and dilution.

\section{Site 63}

Holes at Site 63 penetrated more than 560 meters of section consisting of 35 meters of marl and clay overlying a very pure nannofossil chalk-ooze. Continuous coring was attempted at Site 63 from the sea floor to a depth of 195 meters. Below 195 meters, cores were taken only about each 100 meters. Measurements of the velocity of the compressional wave were made on almost all core sections with the shipboard velocimeter. The compressional wave velocity of seven indurated samples from Site 63 were measured with the University of Hawaii velocimeter. Results are shown in Table 7.

Visual inspection of cores recovered at this site show that lithification increases with depth and that parts or all of the many cores were badly disturbed by the coring process. Maximum values of shear velocity at 180 meters, 225 meters and at 550 meters confirm an increase in rigidity with depth comparable to that at Site 62 . However, most core sections have no rigidity and no shear wave velocity, and this lack is almost certainly due to destruction of rigidity by the coring process. This disturbance also makes compressional wave velocity measurements questionable. Thus, only the maximum values in any given interval should be considered indicative of in-situ values. Based only on these selected values which are indicative of in-situ conditions, the elastic properties of sediments at Site 63 can be summarized as follows.

Generally, the velocity $\left[\mathrm{V}_{\mathrm{p}(\max .)}\right]$ increases exponentially with depth. However, the rate of increase drops between 180 and 350 meters, then increases again to the total depth. The velocity $\left[\mathrm{V}_{\mathrm{p}(\max .)]}\right.$ in the near-surface clay is about 1.45 $\mathrm{km} / \mathrm{sec}$ and in the underlying nannofossil ooze at 65 meters about $1.57 \mathrm{~km} / \mathrm{sec}$. At 165 meters $\mathrm{V}_{\mathrm{p}(\max .)}$ is as high as $1.77 \mathrm{~km} / \mathrm{sec}$ (sample), but a value of 1.6 to $1.7 \mathrm{~km} / \mathrm{sec}$ may be more representative of the whole rock. At 350 meters, one measurement is as high as $2.41 \mathrm{~km} / \mathrm{sec}$, but a value of $1.9 \mathrm{~km} / \mathrm{sec}$ may be more representative. At 560 meters, one measurement as high as $2.44 \mathrm{~km} / \mathrm{sec}$ was made on the shipboard velocimeter. The velocity of a sample from this interval measured at the University of Hawaii is $2.5 \mathrm{~km} / \mathrm{sec}$. The limestone at this depth is well indurated and velocities $\left[\mathrm{V}_{\mathrm{p}(\max .)}\right]$ in the range 2.4 to 2.5 are probably indicative of in-situ values.

Because of the disturbance of the cores at Site 63, no shear wave velocity could be determined. A few derived values, however, may be indicative of in-situ conditions:

A core section at a depth of 2 meters shows a rigidity of 0.03 dynes $/ \mathrm{cm}^{2} \times 1010$ and a shear wave velocity of $0.14 \mathrm{~km} / \mathrm{sec}$. A section at 63 meters shows a rigidity of 0.20 dynes $/ \mathrm{cm}^{2} \times 10^{10}$ and a shear wave velocity of $0.35 \mathrm{~km} / \mathrm{sec}$. Several sections near 180 meters show a rigidity of about 0.85 dynes $/ \mathrm{cm}^{2} \times 10^{10}$ and a shear wave velocity of about $0.7 \mathrm{~km} / \mathrm{sec}$. One section at 232 
TABLE 7

Measurements of Velocity of Compressional Wave, University of Hawaii, for Samples from Site 63

\begin{tabular}{cccclll}
\hline Hole & Core & Section & $\begin{array}{c}\text { Interval } \\
\text { (cm) }\end{array}$ & \multicolumn{1}{c}{ Lithology } & $\begin{array}{c}\text { Compressional } \\
\text { Wave Velocity } \\
\mathrm{km} / \mathrm{sec}\end{array}$ & \multicolumn{1}{c}{ Note } \\
\hline 63.0 & 8 & 1 & $113-119$ & Pink chalk & 2.114 & Perpendicular to bedding \\
63.0 & 8 & 1 & $113-119$ & Pink chalk & 2.402 & Parallel to bedding \\
63.0 & 9 & 1 & $136-142$ & Nannofossil marl & 2.223 & Perpendicular to bedding \\
63.0 & 9 & 1 & $136-142$ & Nannofossil marl & 2.290 & Parallel to bedding \\
63.0 & 9 & 3 & $116-123$ & Nannofossil marl & 2.326 & Perpendicular to bedding \\
63.0 & 9 & 3 & $116-123$ & Nannofossil marl & 2.499 & Parallel to bedding \\
63.1 & 10 & 3 & $17-21$ & Nannofossil chalk & 1.766 & Perpendicular to bedding \\
63.1 & 10 & 3 & $17-21$ & Nannofossil chalk & 1.630 & Parallel to bedding \\
63.1 & 11 & 2 & $107-111$ & Nannofossil chalk & 1.704 & Perpendicular to bedding \\
63.1 & 11 & 2 & $107-111$ & Nannofossil chalk & 1.623 & Parallel to bedding \\
63.1 & 12 & 2 & $45-50$ & Soft nannofossil chalk & 1.680 & Perpendicular to bedding \\
63.1 & 12 & 2 & $45-50$ & Soft nannofossil chalk & 1.658 & Parallel to bedding \\
63.1 & 13 & 6 & $78-82$ & Soft nannofossil chalk & 1.702 & Perpendicular to bedding \\
63.1 & 13 & 6 & $78-82$ & Soft nannofossil chalk & 1.728 & Parallel to bedding \\
\hline
\end{tabular}

meters showed a rigidity as high as 2.19 dynes $/ \mathrm{cm}^{2} \times$ 1010 and a shear wave velocity of $1.08 \mathrm{~km} / \mathrm{sec}$, but a rigidity value of about 1 dyne $/ \mathrm{cm}^{2} \times 1010$ and a shear wave velocity of about $0.76 \mathrm{~km} / \mathrm{sec}$ may be more characteristic of the total rock. At 560 meters, one section showed a rigidity of 3.2 dynes $/ \mathrm{cm}^{2} \times 10^{10}$ and a sonic velocity of $1.25 \mathrm{~km} / \mathrm{sec}$.

These values indicate that the shear wave velocity increases with depth and that the rate of increase decreases with depth.

Valid sonic velocity data collected at this site are too sparse to permit detection of changes in impedance which might account for reflectors noted on seismic profiles.

\section{Site 64}

Holes at Site 64 penetrated almost 1 kilometer of very pure nannofossil chalk ooze. Except for the interval between 430 and 480 meters, Site 64 was cored only at intervals of 50 meters or greater. Compressional wave velocity measurements were made on almost all sections recovered.

The sonic velocity of twelve samples from Site 64 were measured with the University of Hawaii velocimeter. Results are shown on Table 8.
Cores recovered in the upper 200 meters, with few exceptions, show compressional velocities less than 1.5 $\mathrm{km} / \mathrm{sec}$. Cores from the upper nine meters show a velocity of about $1.45 \mathrm{~km} / \mathrm{sec}$. Cores below 200 meters show an irregular exponential increase in compressional wave velocity with depth. Examples of maximum values are: $1.72 \mathrm{~km} / \mathrm{sec}$ at 510 meters, $2.09 \mathrm{~km} / \mathrm{sec}$ at 710 meters, and $2.24 \mathrm{~km} / \mathrm{sec}$ at 850 meters. University of Hawaii measurements on samples of a core from about 850 meters show a compressional wave velocity of $2.53 \mathrm{~km} / \mathrm{sec}$. On samples of silicified limestone from 985 meters velocities range from 4.05 to $4.50 \mathrm{~km} / \mathrm{sec}$.

Coring disturbances appear to have destroyed any original rigidity in a number of cores studied, and no estimate of shear wave velocity of $i$-situ equivalents is possible in these cases. The most reliable values are summarized here. A section from 312 meters shows a rigidity of 0.073 dynes $/ \mathrm{cm}^{2} \times 10^{10}$ and a shear wave velocity of $0.206 \mathrm{~km} / \mathrm{sec}$. A section from 411 meters shows a rigidity of 0.618 dynes $/ \mathrm{cm}^{2} \times 10^{10}$ and a shear wave velocity of $0.601 \mathrm{~km} / \mathrm{sec}$. A section from 710 meters shows a rigidity of 2.00 dynes $/ \mathrm{cm}^{2} \times$ 1010 , and a shear wave velocity of $1.02 \mathrm{~km} / \mathrm{sec}$. A section from 850 meters shows a rigidity of 2.83 dynes $/ \mathrm{cm}^{2} \times 10^{10}$ and a shear wave velocity of 1.21 $\mathrm{km} / \mathrm{sec}$. Materials deeper than 850 meters show indications of core disturbance. 
TABLE 8

Measurements of Velocity of Compressional Wave, University of Hawaii, for Samples from Site 64

\begin{tabular}{|c|c|c|c|c|c|c|}
\hline Hole & Core & Section & $\begin{array}{l}\text { Interval } \\
(\mathrm{cm})\end{array}$ & Lithology & $\begin{array}{c}\text { Compressional } \\
\text { Wave Velocity } \\
\mathrm{km} / \mathrm{sec}\end{array}$ & Note \\
\hline 64.0 & 7 & 1 & 40 & $\begin{array}{l}\text { Chalk } \\
\text { limestone }\end{array}$ & 1.806 & \\
\hline 64.0 & 10 & 1 & $27-28$ & $\begin{array}{l}\text { Chalk } \\
\text { limestone }\end{array}$ & 2.260 & \\
\hline 64.0 & 10 & 1 & $82-83$ & $\begin{array}{l}\text { Chalk } \\
\text { limestone }\end{array}$ & 2.196 & \\
\hline 64.0 & 10 & 1 & $82-83$ & $\begin{array}{l}\text { Chalk } \\
\text { limestone }\end{array}$ & 2.050 & \\
\hline 64.0 & 10 & 2 & $36-37$ & $\begin{array}{l}\text { Chalk } \\
\text { limestone }\end{array}$ & 2.533 & \\
\hline 64.1 & 1 & 4 & & Chalk & 1.929 & $\begin{array}{l}\text { Perpendicular } \\
\text { to bedding }\end{array}$ \\
\hline 64.1 & 1 & 4 & & Chalk & 1.740 & $\begin{array}{l}\text { Parallel to } \\
\text { bedding }\end{array}$ \\
\hline 64.1 & 6 & 2 & & $\begin{array}{l}\text { Chalk } \\
\text { limestone }\end{array}$ & 1.968 & $\begin{array}{l}\text { Perpendicular } \\
\text { to bedding }\end{array}$ \\
\hline 64.1 & 6 & 2 & & $\begin{array}{l}\text { Chalk } \\
\text { limestone }\end{array}$ & 2.110 & $\begin{array}{l}\text { Parallel to } \\
\text { bedding }\end{array}$ \\
\hline 64.1 & 7 & 1 & & $\begin{array}{l}\text { Chalk } \\
\text { limestone }\end{array}$ & 2.177 & $\begin{array}{l}\text { Perpendicular } \\
\text { to bedding }\end{array}$ \\
\hline 64.1 & 7 & 1 & & $\begin{array}{l}\text { Chalk } \\
\text { limestone }\end{array}$ & 1.950 & $\begin{array}{l}\text { Perpendicular } \\
\text { to bedding }\end{array}$ \\
\hline 64.1 & 7 & 1 & & $\begin{array}{l}\text { Chalk } \\
\text { limestone }\end{array}$ & 2.069 & $\begin{array}{l}\text { Parallel to } \\
\text { bedding }\end{array}$ \\
\hline 64.1 & 7 & $\mathrm{CC}$ & & $\begin{array}{l}\text { Chalk } \\
\text { limestone }\end{array}$ & 1.928 & \\
\hline 64.1 & 10 & 1 & & $\begin{array}{l}\text { Chalk } \\
\text { limestone }\end{array}$ & 2.061 & \\
\hline 64.1 & 11 & $\mathrm{CC}$ & & $\begin{array}{l}\text { Silicified } \\
\text { limestone }\end{array}$ & 4.179 & $\begin{array}{l}\text { Perpendicular } \\
\text { to bedding }\end{array}$ \\
\hline 64.1 & 11 & $\mathrm{CC}$ & & $\begin{array}{l}\text { Silicified } \\
\text { limestone }\end{array}$ & 4.048 & $\begin{array}{l}\text { Parallel to } \\
\text { bedding }\end{array}$ \\
\hline 64.1 & 11 & $\mathrm{CC}$ & & $\begin{array}{l}\text { Silicified } \\
\text { limestone }\end{array}$ & 4.498 & $\begin{array}{l}\text { Perpendicular } \\
\text { to bedding }\end{array}$ \\
\hline
\end{tabular}

Site 65

Holes at Site 65 penetrated 185 meters of radiolarian ooze, with some interbedded calcareous ooze below 130 meters. Continuous coring was attempted, and good coverage of measurements of the velocity of the compressional wave were obtained using the shipboard velocimeter. Measurements of compressional wave velocity of the cores are low throughout. The highest velocity measured is $1.54 \mathrm{~km} / \mathrm{sec}$, and most values are less than $1.5 \mathrm{~km} / \mathrm{sec}$.

Near the bottom of Hole 65.1 chert layers were encountered, and brown chert fragments were recovered in the core catcher of 65.1-6 (about 168 
meters below the sea floor). The velocity of the compressional wave of one of these samples was measured at the University of Hawaii. The compressional wave velocity perpendicular to the bedding is $3.457 \mathrm{~km} / \mathrm{sec}$; the velocity parallel to the bedding is $3.736 \mathrm{~km} / \mathrm{sec}$. The saturated bulk density of chert fragments from this core catcher sample ranged from 2.17 to $2.50 \mathrm{gm} / \mathrm{cm}^{2}$.

The acoustic impedance of this chert layer $\left(9000 \mathrm{gm} / \mathrm{cm}^{2} \mathrm{sec} \times 10^{2}\right)$ constitutes a marked change from the impedance of the overlying radiolarian ooze $\left(1700 \mathrm{gm} / \mathrm{cm}^{2} \mathrm{sec} \times 10^{2}\right)$ and this change probably accounts for the reflector on seismic profiles at 0.11 seconds. An increase in drilling rate was also noted at 163 meters (driller's depth).

Gealy and Gerard (1970) found that radiolarian ooze is subject to considerable rebound upon being removed from in-situ pressures. Because of this and the inherently high porosity, measurable rigidity is nil in these cores, and shear wave velocity was determinable on only two core sections.

The calcareous beds near 125 meters are more dense than the radiolarian ooze. Acoustic impedance increases abruptly at this level and may cause the reflection seen on the seismic profile at 0.086 seconds. Also, an increase in drilling rate was noted at 127 meters (driller's depth).

\section{Site 66}

Holtes at Site 66 penetrated about 160 meters of radiolarian ooze overlying a pelagic clay, the sediment overlying a basalt. About forty per cent of the section was cored and measurements of compressional wave velocity were made on most of these with the shipboard velocimeter.
The sonic velocity of one sample of pelagic clay and one sample of basalt from Site 66 were measured with the University of Hawaii velocimeter. Results are shown in Table 9.

As at Site 65, compressional wave velocities $\left[\mathrm{V}_{\mathrm{p}}(\max ).\right]$ in the radiolarian ooze are quite low, ranging from about 1.45 to $1.5 \mathrm{~km} / \mathrm{sec}$. A few measurements on cores near 180 meters showed maximum values as low as 1.41 , but these are probably erroneous.

The pelagic clay has a higher saturated bulk density than the radiolarian ooze $\left(\rho_{\mathrm{B}}=1.6 \mathrm{gm} / \mathrm{cm}^{3}\right.$ versus 1.1 $\mathrm{gm} / \mathrm{cm}^{3}$ ) and also has a higher computed bulk modulus $\left(\mathrm{K}=3.6\right.$ dynes $/ \mathrm{cm}^{2} \times 10^{10}$ versus $2.7 \mathrm{dynes} / \mathrm{cm}^{2} \times$ $10^{10}$ ), yet measurements of compressional wave velocity were lower $\left[\mathrm{V}_{\mathrm{p}}(\max )=.1.45 \mathrm{~km} / \mathrm{sec}\right]$ than those of the overlying ooze $\left[\mathrm{V}_{\mathrm{p}}(\max )=.1.50 \mathrm{~km} / \mathrm{sec}\right]$. Why this is the case is not known, but there is no reason to believe that measurements in either material are invalid.

Data indicate that cores recovered from Site 66 have a residual rigidity too low to be determined by these techniques and no shear wave velocity could be determined.

\section{Site 67}

Core recovery was poor at Site 67 and recovered materials were too badly disturbed to measure sonic velocity with the shipboard velocimeter. However, the velocity of the compressional wave of seven samples from three cores from Site 67 were measured with the University of Hawaii velocimeter. Results are shown on Table 10.

TABLE 9

Measurements of Velocity Compressional Wave, Samples from Site 66 (University of Hawaii)

\begin{tabular}{cccccl}
\hline Hole & Core & Section & Lithology & $\begin{array}{c}\text { Compressional } \\
\text { Wave Velocity } \\
\text { km/sec }\end{array}$ & Note \\
\hline 66.0 & 7 & 3 & $\begin{array}{l}\text { Stiff pelagic } \\
\text { clay } \\
\text { Stiff pelagic } \\
\text { clay } \\
\text { Altered basalt }\end{array}$ & 1.613 & $\begin{array}{l}\text { Perpendicular } \\
\text { to bedding } \\
\text { Parallel to } \\
\text { bedding } \\
\text { Perpendicular } \\
\text { to bedding } \\
\text { Parallel to } \\
\text { bedding }\end{array}$ \\
\hline
\end{tabular}


TABLE 10

Measurements of Velocity of Compressional Wave, Samples from Site 67 (University of Hawaii)

\begin{tabular}{|c|c|c|c|c|c|c|}
\hline Hole & Core & Section & $\begin{array}{c}\text { Interval } \\
(\mathrm{cm})\end{array}$ & Lithology & $\begin{array}{c}\text { Compressional } \\
\text { Wave Velocity } \\
\mathrm{km} / \mathrm{sec}\end{array}$ & Note \\
\hline 67.0 & 1 & 1 & 143 & & 2.188 & Volcanic sandstone \\
\hline 67.0 & 1 & 1 & 145 & & 1.997 & Volcanic mudstone and volcanic sandstone \\
\hline 67.1 & 1 & $\mathrm{CC}$ & & & 1.409 & Volcanic sandstone (a. direction) \\
\hline 67.1 & 1 & $\mathrm{CC}$ & & & 1.564 & Volcanic sandstone (b. direction) \\
\hline 67.1 & 1 & $\mathrm{CC}$ & & & 1.920 & Volcanic sandstone (a. direction) \\
\hline 67.1 & 1 & $\mathrm{CC}$ & & & 2.255 & Volcanic sandstone (b. direction) \\
\hline 67.1 & 2 & $\mathrm{CC}$ & & & 2.742 & Chert \\
\hline
\end{tabular}

\section{DISCUSSION}

\section{Velocity Gradients in Pelagic Sediments}

Calcareous ooze chalk limestone sequences at Sites 62, 63 and 64 show an irregular exponential increase in compressional wave velocity with depth. Compressional wave velocities are consistently less at Site 64 than at Site 62 and Site 63 (Figure 11). The velocity of the shear wave at all three sites increases with depth. Shear wave velocities at Site 64 are lower throughout than velocities at similar depths at Sites 62 and 63 (Figure 12).

Insufficient reliable data were collected on this Leg to determine velocity gradients in either the radiolarian ooze or the pelagic clay.

Valid measurements of compressional wave velocities are higher throughout than can be inferred from the Wood equation (Wood 1941):

$$
\mathrm{V}_{\mathrm{P}}=\sqrt{\frac{\mathrm{K}}{\rho_{\mathrm{B}}}}
$$

These high values result from the increase in rigidity with depth that is so marked at Sites 62,63 , and 64 . All marine sediments probably have some rigidity, although values may be too low to measure or to derive by techniques used here.

\section{Low Velocity Channel}

At all sites occupied on Leg 7, near surface sediments showed a compressional wave velocity less than that of seawater. At Site 62, this low velocity layer is between 20 and 30 meters thick. Because many of the cores are disturbed at Site 63 , it is difficult to determine the thickness of the low velocity layer. The layer at Site 63 is certainly thicker than 10 meters and thinner than 60 meters, and is probably 20 to 30 meters thick as at Site 62. At Site 64 , only cores 300 meters and deeper show velocities consistently higher than seawater, and all of the cores retrieved from Sites 65 and 66 show compressional wave velocities less than that of seawater. Because of core disturbances, it is not possible to determine the thickness of the low velocity layer at any of these three sites.

The low velocity layer has also been observed by others. Fry and Raitt (1961) note a sediment layer of lower sound velocity than that of the overlying water in the Pacific. Shumway (1960) notes that velocities for shallow water silts were about 0.978 that of seawater, and for deep sea red clays were as low as 0.98 that of seawater. Hamilton (1956) found in situ measurements of comparessional wave velocity of unusually fine-grained high porosity sediments to be 2 to 3 per cent less than the velocity of sound in water. Hamilton (1970) discussed low velocity channels in surficial sediments.

Velocity measurements at Sites 62,63 and 64 also show a reversal in the orderly increase with depth. A pronounced reversal is noted at Site 62 between 220 and 240 meters. At Site 63, cores between 70 and 130 meters have velocities lower than cores from 65 meters. However, the degree to which these reversals are due to drilling disturbance is uncertain.

\section{Relationship Between Compressional Wave Velocity and Clay Size}

It has been noted by Hamilton et al. (1966), Shumway (1960), Sutton, et al. (1957), Schreiber (1968), Horn et al. (1968), and others that a relationship exists between grain size parameters and sonic velocity. Hardin and Richart (1963) and Schon (1963) point out that this relationship derives primarily from the relationship that both of these parameters have with porosity. 
The velocity of the compressional wave is dominated by saturated bulk density, compressibility and the rigidity of the sediment; and, a relationship between $\mathrm{V}_{\mathrm{p}}$ and grain size should only be apparent when a comparison is made of pairs of samples which differ only in grain size, and are identical in these other parameters. Relationships between grain size and velocity $\left(\mathrm{V}_{\mathrm{p}}\right)$ may exist only in unconsolidated sediments, and lithification may destroy a preexisting relationship.

Complete grain-size distribution curves are lacking for samples collected on Leg 7, and percentage clay size by weight as reported in Gealy (1971c) is used.

Arrays of values of $\mathrm{V}_{\mathrm{p}(\max )}$ and percentage clay size by weight were compared for data from Sites 62,63 , 64, 65 and 66, and these are shown on Figure 7. A least squares regression of the data yeilded an equation of the form

$$
\text { where } \quad \begin{aligned}
\mathrm{Y} & =\mathrm{AX}+\mathrm{B} \\
\mathrm{X} & =\text { fraction clay size } \\
\mathrm{Y} & =\text { velocity compressional wave in } \mathrm{km} / \mathrm{sec} .
\end{aligned}
$$

The standard deviation of A and B, and the standard error of estimate for $\mathrm{X}$ and for $\mathrm{Y}$ were determined, and shown in Table 11.

Despite the wide variation in saturated bulk density and rigidity, data indicate that samples at all sites show a decrease in compressional wave velocity with increase in percentage clay size. Samples from Sites 62,63 and 64 , as expected, show wide scatter, and this scatter undoubtedly reflects the wide range in cementation encountered at these three sites. Samples from Sites 65 and 66 , on the other hand, show a close relationship between the two arrays with low standard deviation. and standard errors of estimate.

\section{Anisotrophy}

For fifteen samples obtained on Leg 7, the velocity of the compressional wave was measured both parallel to the bedding $\left(\mathrm{V}_{\mathrm{pH}}\right)$ and perpendicular to the bedding $\left(\mathrm{V}_{\mathrm{pV}}\right)$, in order to determine the sense and degree of any anisotrophy in the sediments (Figure 8).

Of the twelve samples of nannofossil chalk ooze from Sites 62,63 and 64 , the ratio $\mathrm{V}_{\mathrm{pH}} / \mathrm{V}_{\mathrm{pV}}$ increases with increasing velocity. Samples having a horizontal velocity less than 2.0 showed a higher vertical velocity than horizontal. Those having a horizontal velocity greater than 2.0 showed a lower vertical velocity than horizontal. If more data confirms such a trend, it may be that in semiconsolidated calcareous sediments, advecting fluids may create a vertical "grain" which could account for this effect in low velocity calcareous sediments. Increased compaction may eventually destroy this vertical grain and crush the material into horizontal layers.

Two samples of chert from radiolarian ooze show higher horizontal velocities than vertical. A sample of silicified limestone shows a lower horizontal velocity than vertical. One sample of stiff pelagic clay from Site 66 shows a higher velocity perpendicular to bedding than parallel to it.

\section{REFERENCES}

Birch, F., 1966. Compressibility: elastic constants in Handbook of Physical Constant. S. P. Clark Jr. (Ed.), Geol. Soc. Amer. Memoir. 97, 98.

Fry, J. C. and Raitt, R. W., 1961. Sound velocity at the surface of deep sea sediments. J. Geophys. Res. 66, 589.

Gassmann, F., 1951. Uberdie lastizitat poroset medien. V. Naturforsch. Ges. Zurich. 96, 1.

Gealy, E. L. (1971a), Saturated bulk density, grain density and porosity of sediments from the Western Equatorial Pacific: Leg VII, Glomar Challenger, In Initial Reports of the Deep Sea Drilling Project, Volume VII, (U. S. Government Printing Office).

Gealy, E. L. (1971b), Carbon-carbonate content of sediments from the Western Equatorial Pacific: Leg VII, Glomar Challenger, In Initial Reports of the Deep Sea Drilling Project, Volume VII, (U.S. Government Printing Office).

Gealy, E. L. (1971c), Grainsize of sediments from the Western Equatorial Pacific: Leg VII, Glomar Challenger, In Initial Reports of the Deep Sea Drilling Project, Volume VII (U.S. Government Printing Office).

Gealy, E. L. and Gerard, R. D. (1970), In situ petrophysical measurements in the Caribbean, In Initial Reports of the Deep Sea Drilling Project, Volume $I V$, Wash., D. C. (U. S. Government Printing Office), p. 269-296.

Hamilton, E. L., 1971. Prediction of in situ acoustic and elastic properties of marine sediments. Geophysics. 36, in press April, 1971.

Hamilton, E. L., 1971. Elastic properties of marine sediments. J. Geophys. Res. 76, 579.

Hamilton, E. L., 1970. Sound velocity and related properties of marine sediments, North Pacific. $J$. Geophys. Res. 75, 4423.

Hamilton, E. L., 1970. Sound channels in surficial marine sediments. J. Acoust. Soc. Am. 48, 1296.

Hamilton, E. L., 1956, Low sound velocities in high-porosity sediments. J. Acoust. Soc. Am. 28, 16.

Hamilton, E. L., Bucker, H. P., Keir, D. L., and Whitney, J. A., 1970. Velocities of compressional and shear waves in marine sediments determined in 

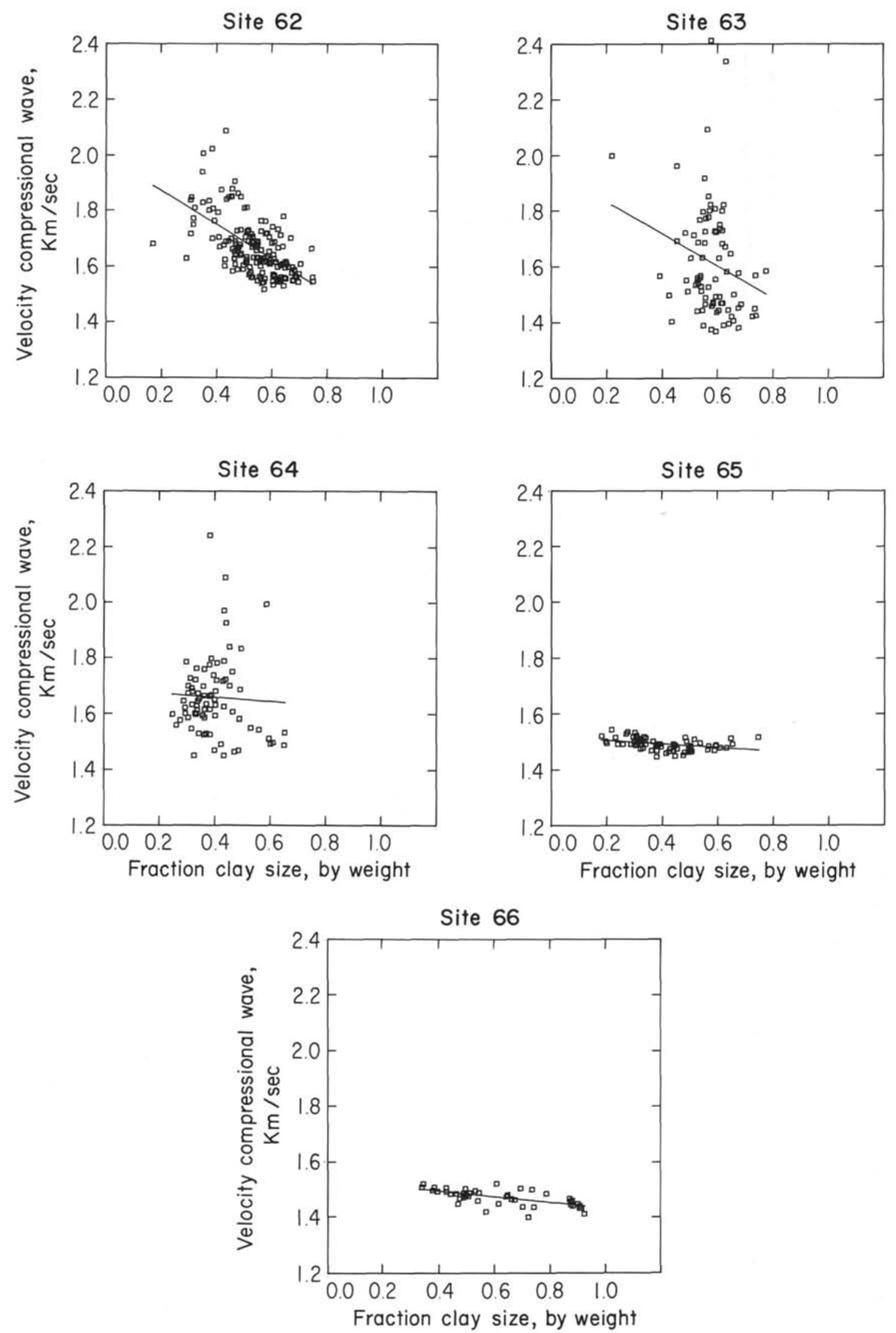

Figure 7. Comparison of velocity of the compressional wave and per cent clay size by weight. 


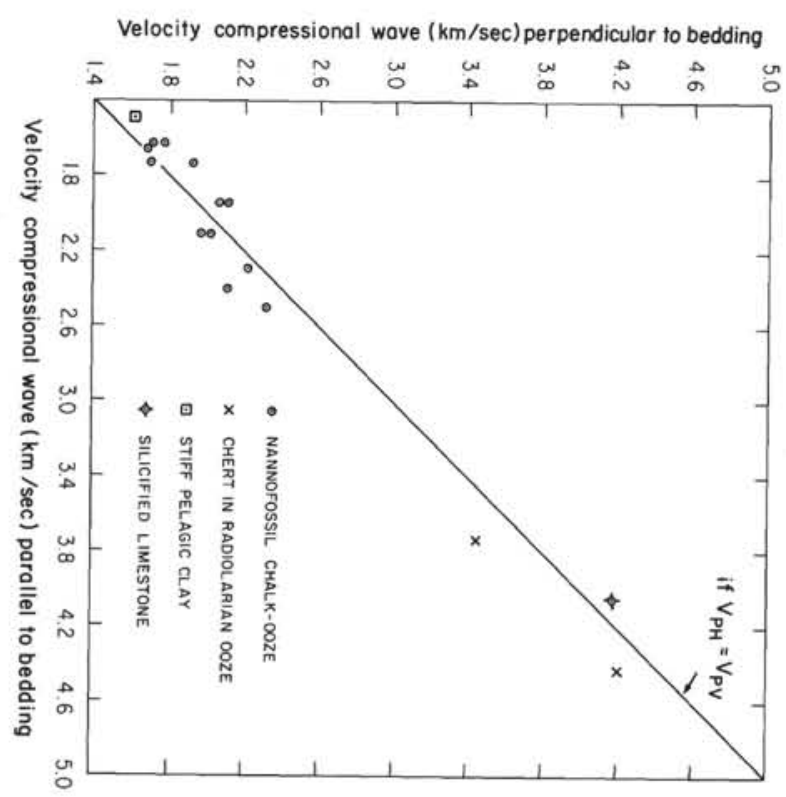

Figure 8. Comparison of velocity of compressional wave measured horizontally and perpendicular to bedding.

situ from a research submersible. J. Geophys. Res. 75, (20) July 10, 1970.

Hardin, B. V. and Richart, Jr., F. E., 1963. Elastic wave velocities in granular soils. J. Soil Mech. Found. Div. Am. Soc. Chem. Engrs. 89, SMI, 33.

Horn, D. R., Horn, B. M., and Delache, M. N., 1968. Correlation between acoustic and other physical properties of deep sea cores. J. Gepphys. Res. 73, 1939.
McManus, Dean, 1970. Comparison of three methods of measuring or estimating sonic velocity in sediments. In Initial Reports of the Deep Sea Drilling Projects, Volume V. Washington (U.S. Government Printing Office) 545.

Molotova, L. V. and Vassil'ev, Y. J., 1960. Velocity ratio of longitudinal and transverse waves in rocks. Bull. (Izv.) Akad. Sci. USSR, Geophys. Serv., English Transl., No. 8, 731.

NAVOCEANO, 1966. Handbook of Oceanographic Tables. U. S. Naval Oceanographic Office. SP-68.

Peselnick, L., 1962. Elastic constants of solenhofen Limestone and their dependence on density and saturation. J. Geophys. Res. 67, 4441.

Peterson, M. N. A. et. al., (1969), Initial Reports of the Deep Sea Drilling Project, Volume II, (U. S. Government Printing Office).

Schon, J., 1963. Modellseismische Untersuchungen im Hinblick auf die Schollgeschwinddigkeit in Lockergesteinen, Monatsber. Deut. Akad. Wiss. Berlin. 5, $4,262$.

Schreiber, B. C., 1968. Sound velocity in deep sea sediments. J. Geophys. Res. 73, 1259.

Shumway, G., 1960. Speed and absorption studies of marine sediments by a resonance method, 1 and 2 . Geophysics. 25, 451-467, 659-682.

Skempton, A. W., 1961. Effective stress in soils, concrete and rocks. In Pore Pressure and Suction in Soils. London (Butterworth).

Sutton, G. N., Berckheimer, H. and Nafe, J. E., 1957. Physical analysis of deep sea sediments. Geophysics. 22, 779 .

Urick, R. J., 1947. A sound velocity method for determining the compressibility of finely divided substances. J. Appl. Phys. 18, 983.

Wood, D. B., 1941. A Textbook of Sound. London (G. Bell), $578 \mathrm{pp}$.

TABLE 11

Comparison of Compressional Wave and Fraction Clay Size (by weight)

\begin{tabular}{|c|c|c|c|c|c|c|c|}
\hline \multirow[b]{2}{*}{ Site } & \multirow[b]{2}{*}{ Lithology } & \multicolumn{2}{|c|}{ A } & \multicolumn{2}{|c|}{ B } & \multicolumn{2}{|c|}{$\begin{array}{l}\text { Standard Error } \\
\text { of Estimate }\end{array}$} \\
\hline & & Value & $\begin{array}{l}\text { Standard } \\
\text { Deviation }\end{array}$ & Value & $\begin{array}{l}\text { Standard } \\
\text { Deviation }\end{array}$ & $\mathrm{X}$ & $\mathrm{Y}$ \\
\hline 62 & Calcareous ooze-chalk & -0.610 & 0.016 & +1.991 & 0.030 & 0.137 & 0.084 \\
\hline 63 & Calcareous ooze-chalk & -0.579 & 0.072 & +1.949 & 0.123 & 0.338 & 0.196 \\
\hline 64 & Calcareous ooze-chalk & -0.071 & 0.034 & +1.685 & 0.082 & 1.900 & 0.138 \\
\hline 65 & Radiolarian ooze & -0.067 & 0.004 & +1.519 & 0.009 & 0.292 & 0.020 \\
\hline 66 & $\begin{array}{l}\text { Radiolarian ooze, } \\
\text { pelagic clay }\end{array}$ & -0.103 & 0.005 & +1.534 & 0.008 & 0.202 & 0.021 \\
\hline
\end{tabular}




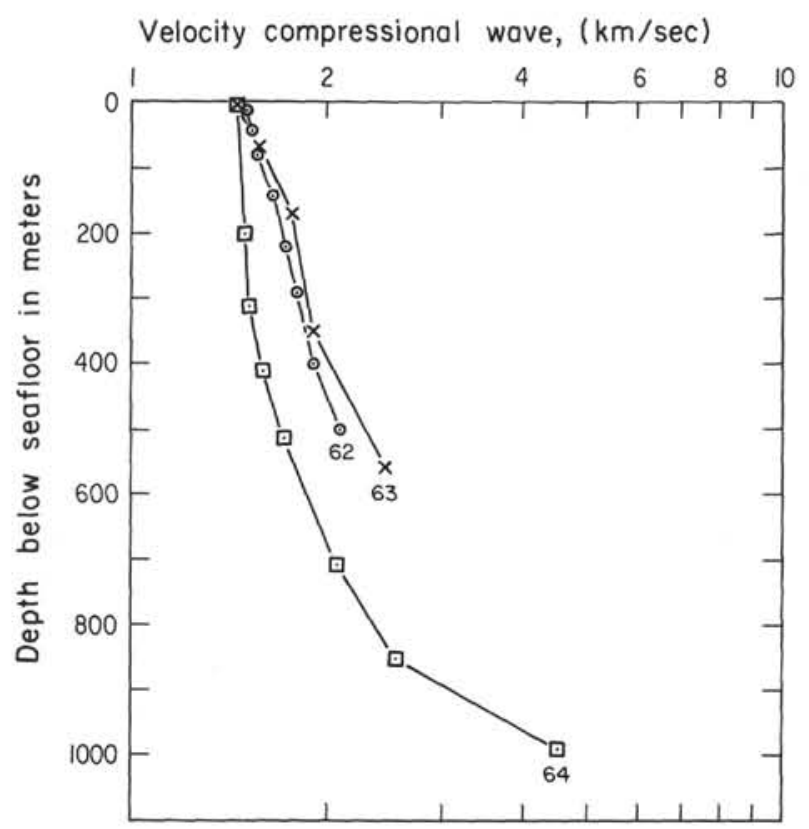

Figure 9. Velocity compressional waves versus depth (Sites 62, 63 and 64).

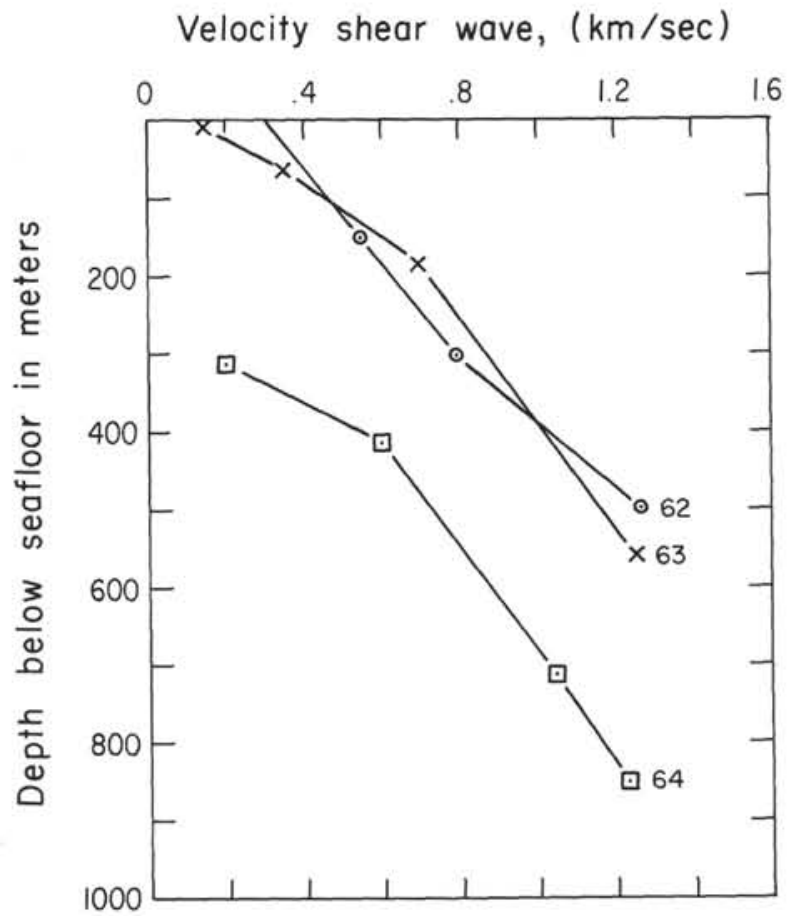

Figure 10. Velocity shear waves versus depth (Sites 62, 63 and 64). 